This Supporting Information for Manuscript:

Tittle: Graphene Oxide as a Carbocatalyst for Sustainable ipso- Hydroxylation of Arylboronic acids: A Simple and Straightforward Strategy to Access Phenols

Authors: Murugan Karthik and Palaniswamy Suresh*

*Email : ghemistry@gmail.com

suersh.chem@mkuniversity.org

Number of pages: 41 (Pages S1-S41)

Number of Tables: 4 (Tables S1-S4)

Number of Figures: 59 (Figures S1-S59)

References: 10 (Ref. S1-S10) 


\title{
Graphene Oxide as a Carbocatalyst for Sustainable ipso- Hydroxylation of Arylboronic acids: A Simple and Straightforward Strategy to Access Phenols
}

\author{
Murugan Karthik ${ }^{\dagger}$, Palaniswamy Suresh*+ \\ ${ }^{+}$Supramolecular and Catalysis Laboratory, Department of Natural Products Chemistry, \\ School of Chemistry, Madurai Kamaraj University, Madurai - 625021. India. \\ E-mail: ghemistry@gmail.com, suresh.chem@mkuniversity.org*
}

\section{Table of Contents}

Preparation and Characterization of Catalyst

Experimental Section

Comparison Table

NMR data

Reference

${ }^{1} \mathrm{H}$ NMR and ${ }^{13} \mathrm{C}$ NMR spectra

S16 


\section{Preparation and Characterization of Catalyst}

Following a reported modified Hummers and Hoffman's method, ${ }^{1}$ GO catalyst was synthesized: typically, equal quantity of graphite $(2.5 \mathrm{~g})$ and $\mathrm{NaNO}_{3}(2.5 \mathrm{~g})$ was dispersed in $\mathrm{H}_{2} \mathrm{SO}_{4}(115 \mathrm{~mL})$ in an ice bath. To this $\mathrm{KMnO}_{4}(15.0 \mathrm{~g})$ was added gradually and it was allowed to stir at room temperature for $12 \mathrm{~h}$. After it was diluted with $500 \mathrm{~mL}$ of DD water, the mixture was heated to $98^{\circ} \mathrm{C}$ for $1 \mathrm{~h}$. Then the reaction was terminated by adding $15 \mathrm{~mL}$ of $30 \%$ aqueous $\mathrm{H}_{2} \mathrm{O}_{2}$ solution followed by washed with $5 \% \mathrm{HCl}$ solution to remove inorganic sulphate impurities. Finally, the resulting graphite oxide was washed and repeatedly dialyzed with DD water. And dried in a vacuum oven at $55^{\circ} \mathrm{C}$ for $24 \mathrm{~h}$ to obtain graphite oxide.

Primarily Energy Dispersive X-ray Spectroscopy (EDS) Fig. S2a reveals the oxidation of graphite as well as lack of metal impurities by showing 74.5\% Carbon and $25.4 \%$ oxygen in the graphitic skeleton. The FT-IR spectrum of GO is shown in Fig. S1b. The stretching vibrations at $3384 \mathrm{~cm}^{-1}, 1727 \mathrm{~cm}^{-1}$ were corresponds to hydroxyl $(\mathrm{OH})$ and carbonyl group $(\mathrm{C}=\mathrm{O})$ present in the graphitic sheet. Also, the spectrum shows the deformation vibration peak of $\mathrm{C}-\mathrm{OH}$ at $1364 \mathrm{~cm}^{-1}$, a weak peak at $1273 \mathrm{~cm}^{-1}$ attributed to the epoxy group and a peak at $1064 \mathrm{~cm}^{-1}$ ascribed to the C-O-C stretching vibrations. ${ }^{2}$ Which is further evident from the X-ray photoelectron spectroscopy (XPS) (Fig S1(c). The deconvoluted C1s pattern of GO shows peaks at 286.1, 287.9 and $290.0 \mathrm{eV}$ which are associated with binding energies of $\mathrm{C}-\mathrm{OH}, \mathrm{C}=\mathrm{O}$, and $\mathrm{O}=\mathrm{C}-\mathrm{OH}$ functional groups respectively.

Scanning Electron Microscopic (SEM) and Transmission Electron Microscopic (TEM) examination of GO shows (Fig. S2a \& 2b) crumpled lamellar flexible sheet like morphology with clear surface area. This may facilitate the high mass transfer of reactant to the products. Powder XRD was also tested and the pattern is shown in Fig. S1d. It was found that ' 002 ' plane of graphite at $26^{\circ}$ shifted to $10.3^{\circ}$ in $\mathrm{GO}$ along with increase in the interplanar distance. This is due to intercalated oxygen functionalities on the graphitic scaffold. 

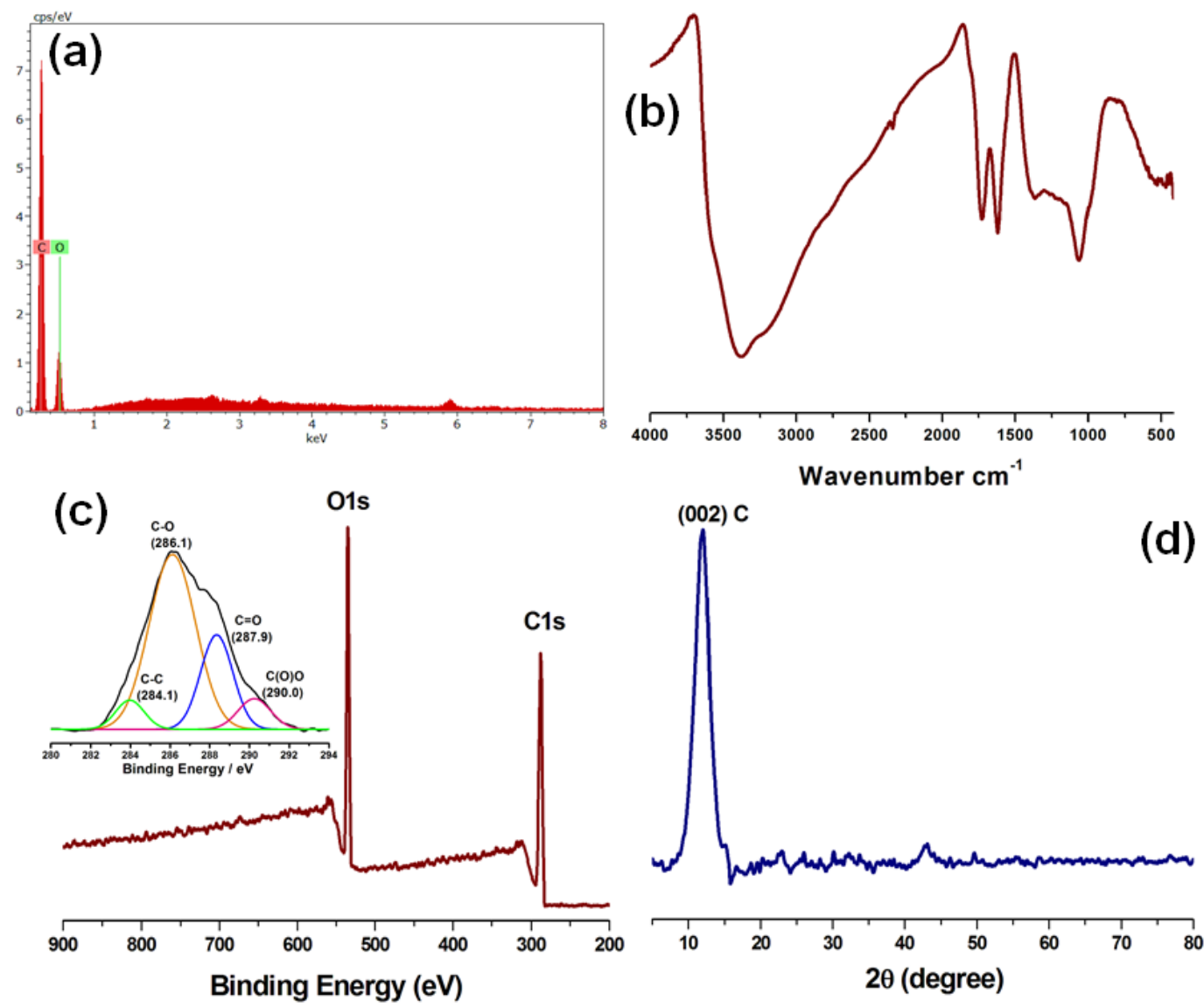

Fig. S1. a) EDS spectrum, b) FT-IR spectrum, c) XPS spectrum (insert deconvoluted C1s Peak), d) PXRD pattern of GO
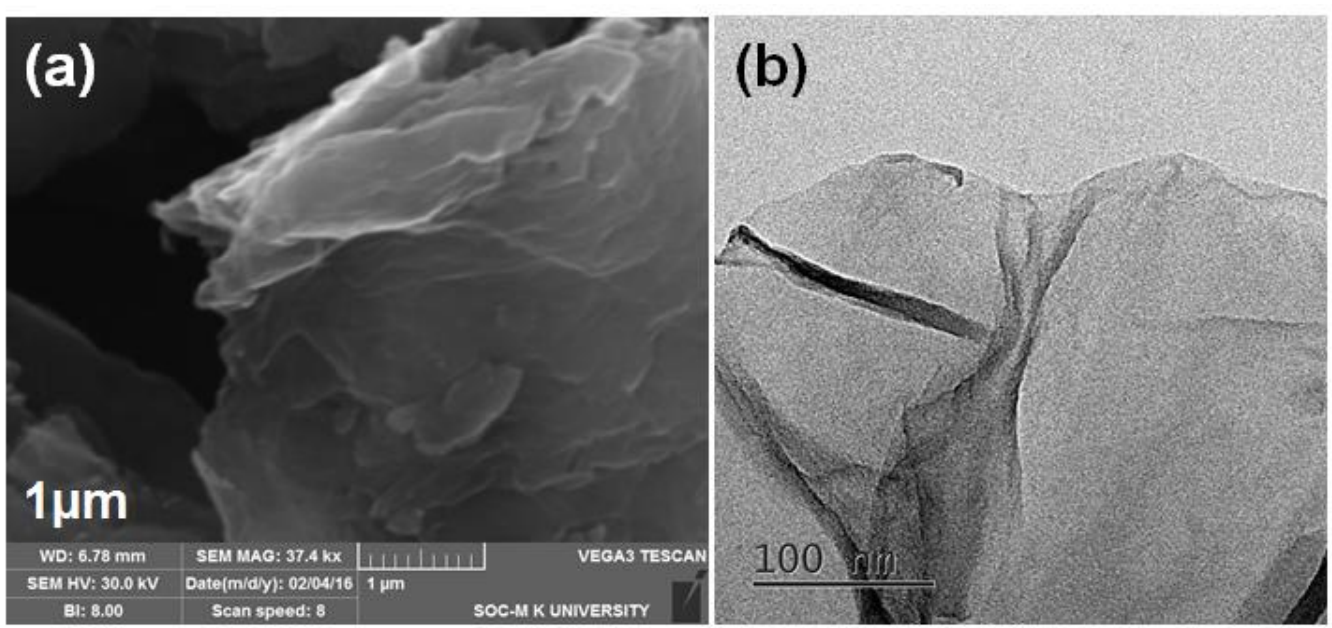

Fig. S2. a) SEM, b) TEM image of GO 


\section{Experimental Section}

\subsection{Optimization of oxidant load on ipso-hydroxylation.}

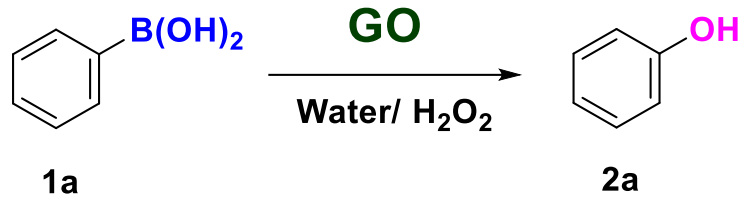

Table S1 Effect of oxidant load ${ }^{a}$

\begin{tabular}{|c|c|c|c|}
\hline \multirow{2}{*}{ Entry } & \multicolumn{2}{|c|}{$30 \% \mathrm{H}_{2} \mathrm{O}_{2}$ Oxidant Load } & \multirow{2}{*}{ Yield $[\%]^{b}$} \\
\hline & (eq) & $(\mathrm{mmol})$ & \\
\hline 1 & $1(12 \mu \mathrm{L})$ & 0.5 & 42 \\
\hline 2 & $2(24 \mu \mathrm{L})$ & 1.0 & 63 \\
\hline 3 & $3(36 \mu \mathrm{L})$ & 1.5 & 76 \\
\hline 4 & $4(48 \mu \mathrm{L})$ & 2.0 & 100 \\
\hline 5 & $5(60 \mu \mathrm{L})$ & 2.5 & 100 \\
\hline 6 & $6(72 \mu \mathrm{L})$ & 3.0 & 100 \\
\hline
\end{tabular}

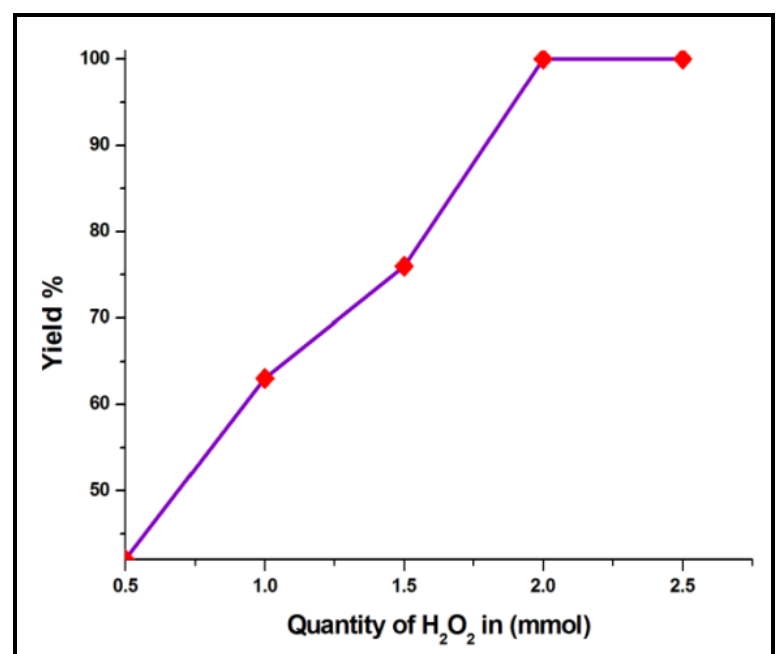

Fig. S3. Oxidant load optimization 


\subsection{Optimization of Catalyst Load on ipso-Hydroxylation.}

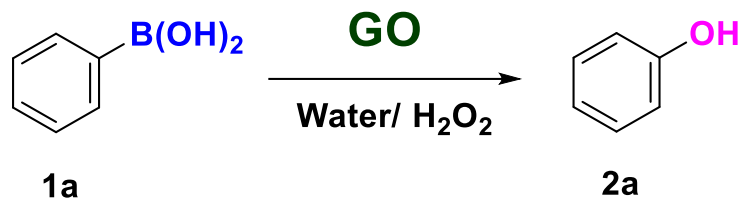

Table S2 Effect of Catalyst Load ${ }^{a}$

\begin{tabular}{|c|c|c|c|}
\hline \multirow{2}{*}{ Entry } & \multicolumn{2}{|c|}{$\begin{array}{c}\text { GO Catalyst } \\
\end{array}$} & \multirow{2}{*}{ Yield $[\%]^{\mathrm{b}}$} \\
\hline & (mg) & $\mathbf{W t}[\%]^{\mathrm{c}}$ & \\
\hline 1 & 5 & 8.2 & 50 \\
\hline 2 & 10 & 16.4 & 84 \\
\hline 3 & 15 & 24.6 & 100 \\
\hline 4 & 20 & 32.8 & 100 \\
\hline 5 & 30 & 49.2 & 100 \\
\hline 6 & 40 & 65.6 & 100 \\
\hline \multicolumn{4}{|c|}{$\begin{array}{l}{ }^{a} \text { Reaction Condition: Phenylboronic acid }(0.5 \mathrm{mmol}), 30 \% \mathrm{H}_{2} \mathrm{O}_{2}(2 \mathrm{mmol}) \text { and } \mathrm{GO} \text { in } 2 \\
\mathrm{~mL} \text { of water at room temperature in } 5 \mathrm{~min} .{ }^{b} \text { isolated yield. }{ }^{c} \mathrm{Wt} \% \text { was calculated with } \\
\text { respect to phenylboronic acid }\end{array}$} \\
\hline
\end{tabular}

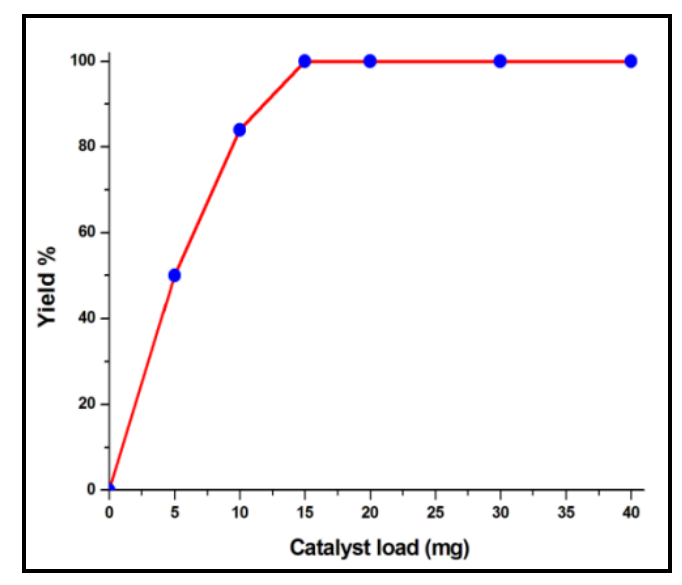

Fig. S4. Catalyst load optimization

\subsection{Typical Procedure for the Synthesis of GO-Ester.}

Methyl ester of GO (GO-Ester) was prepared by adopting the reported Steglich esterification procedure. ${ }^{3}$ To a dispersed solution of $\mathrm{GO}(500 \mathrm{mg})$ in $300 \mathrm{~mL}$ anhydrous methanol, $100 \mathrm{mg}$ of 4-dimethylaminopyridine (DMAP) was added. Followed by dicyclohexylcarbodiimide (DCC) was added to the reaction mixture at $0^{\circ} \mathrm{C}$ in an ice bath, which was then allowed to stir for $5 \mathrm{~min}$. Further the temperature raised to $20^{\circ} \mathrm{C}$ and continued to stir for $3 \mathrm{~h}$. The resulting black solid was separated by the filtration and washed twice with $0.05 \mathrm{~N} \mathrm{HCl}$ followed by saturated $\mathrm{NaHCO}_{3}$ 
solution. Finally, the formed methyl ester of GO was washed with excess of cold water and then dialysed for $10 \mathrm{~h}$ in order to remove any metal contaminants, if present. The final product was dried in vacuum oven for $24 \mathrm{~h}$ at $60{ }^{\circ} \mathrm{C}$ and characterised using FT-IR analysis.

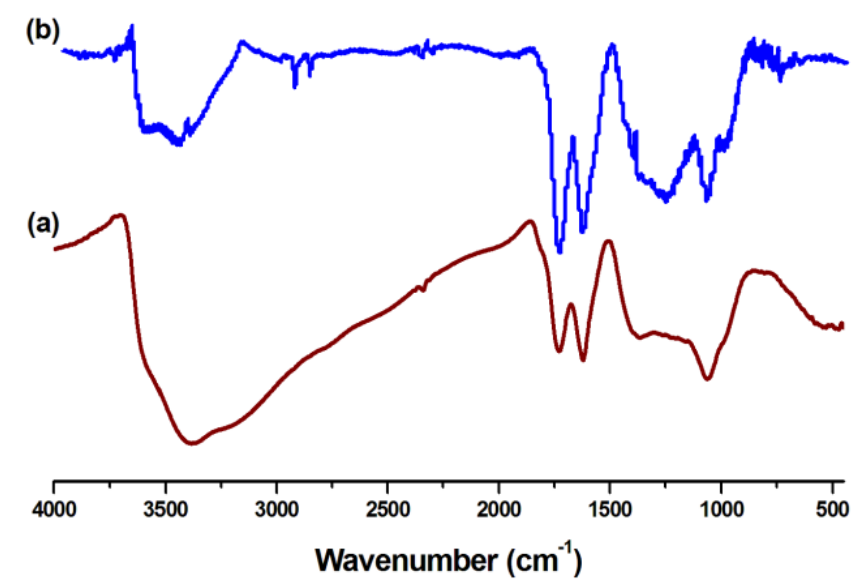

Fig. S5. FT-IR spectra of a) GO, b) Methyl ester of GO (GO-Ester)

\section{3a Characterization of GO-Ester}

Chemical transformation of carboxylic acid into methyl ester of GO was envisaged by Fourier Transform Infrared (FTIR) spectroscopy. The bands at 1730 and $1250 \mathrm{~cm}^{-1}$ in figure $\mathrm{S} 5 \mathrm{~b}$ attributed to the $\mathrm{C}=\mathrm{O}$ stretching and the $\mathrm{C}-\mathrm{O}$ stretching of the ester groups, which reveals the formation of GO methyl ester via covalent bonding between graphene oxide and $\mathrm{CH}_{3} \mathrm{OH}$. Besides, the presence of $\mathrm{CH}_{3} \mathrm{O}$-group is confirmed by the appearance of two absorption bands at 2920, and $2852 \mathrm{~cm}^{-1}$ associated with aliphatic $\mathrm{CH}$ stretching of methyl group. rest of the bands were resembling the GO pattern.

\subsection{Typical Procedure for the Catalyst Reuse:}

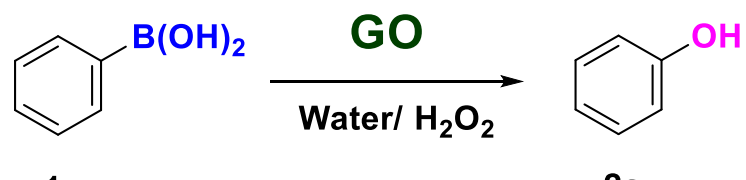

$1 \mathrm{a}$

$2 a$

In an oven dried reaction tube, phenylboronic acid (1a, $0.5 \mathrm{mmol}), 15 \mathrm{mg} \mathrm{GO}$ (24 Wt. \%), and $2 \mathrm{~mL}$ of water taken followed by $\mathrm{H}_{2} \mathrm{O}_{2}(2 \mathrm{mmol}, 4$ eq) was added dropwise. The mixture was stirred at room temperature for 5 mins. After completion 
of the reaction, product was separated using ethyl acetate. Next the catalyst was removed by simple entrifugation and washed with water and then dried under vacuum at $50{ }^{\circ} \mathrm{C}$. The recovered catalyst was reused for the next cycle. Before recycling, the stability of the catalyst was characterized using FTIR, PXRD and TEM (Fig S 6-7).

Table S3 Recycling of the GO Catalyst ${ }^{a}$

\begin{tabular}{lcccccc}
\hline Run & Fresh & $\mathbf{1}$ & $\mathbf{2}$ & $\mathbf{3}$ & $\mathbf{4}$ & $\mathbf{5}$ \\
\hline Yield $(\%)^{b}$ & 99 & 99 & 98 & 97 & 97 & 97
\end{tabular}

${ }^{a}$ Reaction Condition: Phenylboronic acid $(0.5 \mathrm{mmol}), \mathrm{H}_{2} \mathrm{O}_{2}(2 \mathrm{mmol})$ and $15 \mathrm{mg}$ of $\mathrm{GO}$ in $2 \mathrm{~mL}$ of water at room temperature in $5 \mathrm{~min} .{ }^{b}$ isolated yield

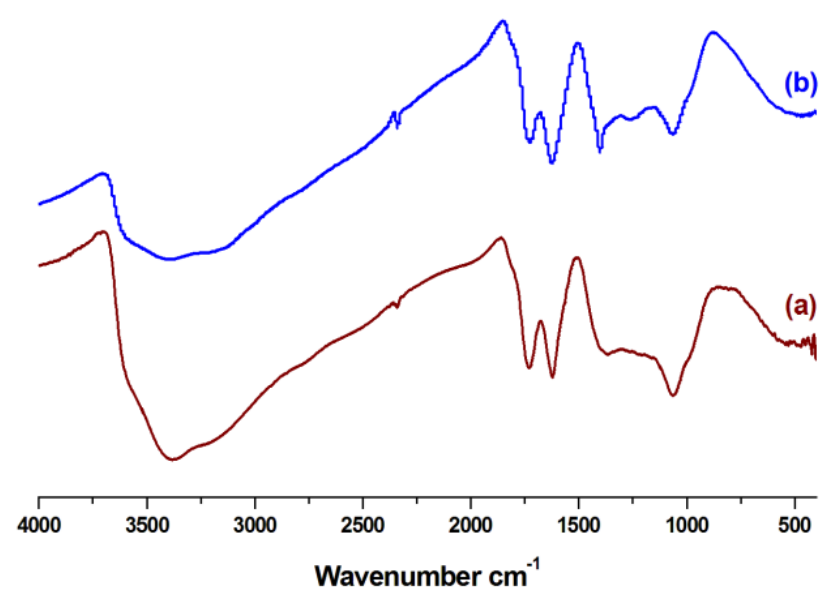

Fig. S6 FTIR pattern of a) GO, b) fifth-time reused-GO
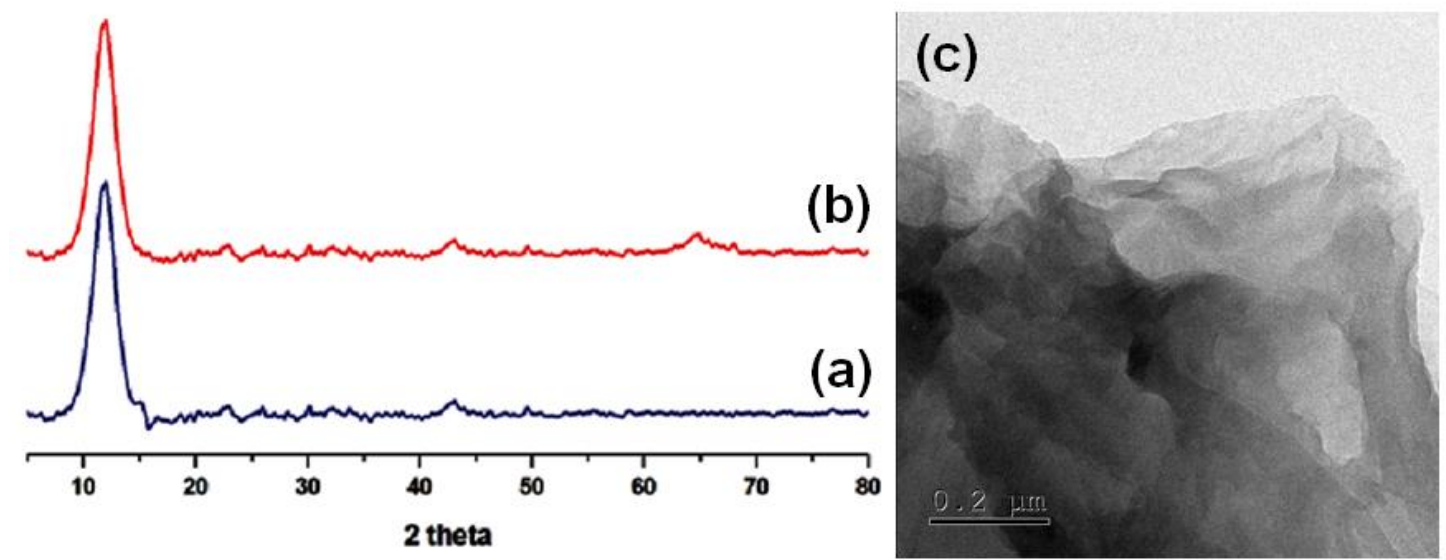

Fig. S7. PXRD pattern of a) GO, b) fifth time reused-GO, \& c) TEM image of fifth time reused GO. 
Table S4 Comparison of GO Catalysed Oxidation Reactions

\begin{tabular}{|c|c|c|c|c|c|c|c|}
\hline \multirow{2}{*}{$\begin{array}{l}\text { S. } \\
\text { No. }\end{array}$} & \multirow{2}{*}{ Reaction } & \multicolumn{2}{|c|}{ GO load } & \multirow{2}{*}{ Oxidant } & \multirow{2}{*}{$\begin{array}{l}\text { Temperature } \\
\qquad\left({ }^{\circ} \mathrm{C}\right)\end{array}$} & \multirow{2}{*}{$\begin{array}{l}\text { Time } \\
\text { (h) }\end{array}$} & \multirow{2}{*}{ Ref. } \\
\hline & & $\mathrm{Wt} \%$ & $\mathrm{mg}$ & & & & \\
\hline 1 & $\begin{array}{l}\text { Oxidation \& Hydration } \\
\text { reactions }\end{array}$ & 200 & - & - & 100 & 24 & 4 \\
\hline 2 & C-H Oxidation & 400 & 200 & - & 100 & 24 & 5 \\
\hline 3 & $\begin{array}{l}\text { Oxidative coupling of amines } \\
\text { to imines }\end{array}$ & 50 & - & $\mathrm{O}_{2}(5 \mathrm{~atm})$ & 100 & 4 & 6 \\
\hline 4 & $\begin{array}{l}\text { Selective Oxidation of } \\
\text { Glutaraldehyde }\end{array}$ & - & 50 & $\mathrm{H}_{2} \mathrm{O}_{2}(50 \%)$ & 90 & 10 & 7 \\
\hline 5 & Oxidation of thioanisole & 10 & 4 & $\mathrm{H}_{2} \mathrm{O}_{2}(30 \%)$ & $20-25$ & 72 & 8 \\
\hline 6 & $\begin{array}{l}\text { Aerobic Oxidation of } \\
\text { 5-Hydroxymethylfurfural into } \\
\text { 2,5-Diformylfuran }\end{array}$ & 80 & 100 & $\begin{array}{l}\text { TEMPO \& } \\
\mathrm{O}_{2}\end{array}$ & 100 & 9 & 9 \\
\hline 7 & $\begin{array}{l}\text { Chemoselective } \\
\text { Alcohol Oxidation }\end{array}$ & 5 & - & $\mathrm{HNO}_{3} \&$ air & 90 & 3 & 10 \\
\hline 8 & $\begin{array}{l}\text { ipso-Hydroxylation of } \\
\text { boronic acid }\end{array}$ & 24.6 & 15 & $\mathrm{H}_{2} \mathrm{O}_{2}(30 \%)$ & rt & $\begin{array}{c}5 \\
(\mathrm{~min})\end{array}$ & $\begin{array}{l}\text { This } \\
\text { work }\end{array}$ \\
\hline
\end{tabular}




\section{NMR DATA}<smiles>Oc1ccccc1</smiles>

Phenol (2a) ${ }^{1} \mathbf{H}$ NMR (300 MHz, $\left.\mathrm{CDCl}_{3}\right) \delta 7.27-7.16(\mathrm{~m}, 2 \mathrm{H}), 6.97-6.88(\mathrm{~m}, 1 \mathrm{H}), 6.87-$ $6.79(\mathrm{~m}, 2 \mathrm{H}), 6.05(\mathrm{~s}, 1 \mathrm{H})$. (Fig. S8); ${ }^{13} \mathbf{C} \mathbf{N M R}\left(75 \mathrm{MHz}, \mathrm{CDCl}_{3}\right) \delta 155.19,129.67$, 120.82,115.33. (Fig. S9)<smiles>Oc1ccc(Cl)cc1</smiles>

4-Chlorophenol (2b) ${ }^{1} \mathbf{H}$ NMR $\left(300 \mathrm{MHz}, \mathrm{CDCl}_{3}\right) \delta 7.18(\mathrm{dd}, J=8.9,2.5 \mathrm{~Hz}, 2 \mathrm{H}), 6.77$ $(\mathrm{dd}, J=8.8,2.5 \mathrm{~Hz}, 2 \mathrm{H}), 5.96$ (s, 1H). (Fig. S10); ${ }^{13} \mathbf{C ~ N M R}\left(75 \mathrm{MHz}, \mathrm{CDCl}_{3}\right) \delta 153.39$, 129.42, 125.60, 116.57. (Fig. S11)<smiles>Oc1ccc(Br)cc1</smiles>

4-Bromophenol (2c) ${ }^{1} \mathbf{H}$ NMR (300 MHz, $\left.\mathrm{CDCl}_{3}\right) \delta 7.33(\mathrm{dd}, J=8.9,2.8 \mathrm{~Hz}, 2 \mathrm{H}), 6.72(\mathrm{dd}$, $J=8.9,2.8 \mathrm{~Hz}, 2 \mathrm{H}), 5.13$ (s, 1H). (Fig. S12); ${ }^{13} \mathbf{C ~ N M R}\left(75 \mathrm{MHz}, \mathrm{CDCl}_{3}\right) \delta 153.83,132.45$, 117.12, 113.16. (Fig. S13)<smiles>Oc1ccc(I)cc1</smiles>

4-Iodophenol (2d) ${ }^{1} \mathbf{H}$ NMR $\left(300 \mathrm{MHz}, \mathrm{CDCl}_{3}\right) \delta 7.65(\mathrm{dd}, J=7.9,1.4 \mathrm{~Hz}, 1 \mathrm{H}), 7.23(\mathrm{td}, J$ $=8.1,1.5 \mathrm{~Hz}, 1 \mathrm{H}), 6.99(\mathrm{dd}, J=8.1,1.4 \mathrm{~Hz}, 1 \mathrm{H}), 6.72-6.62(\mathrm{~m}, 1 \mathrm{H}), 5.35(\mathrm{~s}, 1 \mathrm{H})$. (Fig. $\mathrm{S} 14) ;{ }^{13} \mathrm{C}$ NMR $\left(75 \mathrm{MHz}, \mathrm{CDCl}_{3}\right) \delta 154.49,138.20,129.99,122.35,115.10$, 85.59. (Fig. S15)<smiles>O=[N+]([O-])c1ccc(O)cc1</smiles>

4-Nitrophenol (2e) ${ }^{\mathbf{1}} \mathbf{H}$ NMR $\left(300 \mathrm{MHz}, \mathrm{CDCl}_{3}\right) \delta 8.18(\mathrm{~d}, J=8.6 \mathrm{~Hz}, 2 \mathrm{H}), 6.93(\mathrm{~d}, J=8.5$ $\mathrm{Hz}, 2 \mathrm{H}), 6.31$ (s, 1H). (Fig. S16); ${ }^{13} \mathbf{C} \mathbf{N M R}\left(75 \mathrm{MHz}, \mathrm{CDCl}_{3}\right) \delta 163.27,140.00,125.75$, 115.38. (Fig. S17)<smiles>Cc1ccc(O)cc1</smiles> 
4-Methylphenol (2f) ${ }^{1} \mathbf{H}$ NMR $\left(300 \mathrm{MHz}, \mathrm{CDCl}_{3}\right) \delta 7.13-6.95(\mathrm{~m}, 2 \mathrm{H}), 6.74(\mathrm{t}, J=8.7 \mathrm{~Hz}$, 2H), 5.54 (s, 1H), 2.27 (d, $J=8.8 \mathrm{~Hz}, 3 \mathrm{H})$. (Fig. S18); ${ }^{13} \mathbf{C ~ N M R}\left(75 \mathrm{MHz}, \mathrm{CDCl}_{3}\right) \delta 153.18$, 130.33, 115.27, 20.56 (Fig. S19).<smiles>COc1ccc(O)cc1</smiles>

4-Methoxyphenol (2g) ${ }^{1} \mathbf{H}$ NMR $\left(300 \mathrm{MHz} \mathrm{CDCl}_{3}\right) \delta 7.00-6.70(\mathrm{~m}, 4 \mathrm{H}), 5.28(\mathrm{~s}, 1 \mathrm{H})$, 3.76 (s, 3H) (Fig. S20); $\left.{ }^{13} \mathbf{C ~ N M R ~ ( 7 5 ~ M H z , ~} \mathrm{CDCl}_{3}\right) \delta 153.75,149.62,116.21,115.03,55.98$. (Fig. S21)<smiles>COc1ccc(O)cc1OC</smiles>

$\underline{\text { 3,4-Dimethoxyphenol (2h) }}{ }^{\mathbf{1}} \mathbf{H}$ NMR (300 MHz, $\left.\mathrm{CDCl}_{3}\right) \delta 6.80-6.66(\mathrm{~m}, 1 \mathrm{H}), 6.52-6.43$ (m, 1H), 6.36 (ddd, $J=8.6,2.8,1.4 \mathrm{~Hz}, 1 \mathrm{H}), 5.54$ (s, 1H), 3.81 (dd, $J=4.9,1.3 \mathrm{~Hz}, 6 \mathrm{H})$. (Fig. S22); ${ }^{13}$ C NMR (75 MHz, $\left.\mathrm{CDCl}_{3}\right) \delta 150.45,149.73,142.75,112.75,106.12,100.75$, 56.63, 55.69. (Fig. S23)<smiles>Cc1ccccc1O</smiles>

2-Methylphenol (2i) ${ }^{1} \mathbf{H}$ NMR (300 MHz, CDCl 3$) \delta 7.09$ (dd, $\left.J=15.4,7.3 \mathrm{~Hz}, 2 \mathrm{H}\right), 6.91-$ $6.67(\mathrm{~m}, 2 \mathrm{H}), 5.03(\mathrm{~s}, 1 \mathrm{H}), 2.24(\mathrm{~d}, J=3.7 \mathrm{~Hz}, 3 \mathrm{H})$. (Fig. S24); ${ }^{13} \mathbf{C} \mathbf{N M R}\left(75 \mathrm{MHz}, \mathrm{CDCl}_{3}\right)$ $\delta 153.63(\mathrm{~s}), 131.01(\mathrm{~d}, J=1.5 \mathrm{~Hz}), 127.01(\mathrm{~d}, J=1.5 \mathrm{~Hz}), 123.96(\mathrm{~d}, J=1.5 \mathrm{~Hz}), 120.72$, 114.94, 15.64. (Fig. S25)<smiles>O=[N+]([O-])c1ccccc1O</smiles>

2-Nitrophenol (2j) ${ }^{\mathbf{1}} \mathbf{H}$ NMR $\left(300 \mathrm{MHz}, \mathrm{CDCl}_{3}\right) \delta 10.60(\mathrm{~s}, 1 \mathrm{H}), 8.11(\mathrm{dd}, J=8.5,1.6 \mathrm{~Hz}$, $1 \mathrm{H}), 7.59$ (ddd, $J=8.6,7.2,1.6 \mathrm{~Hz}, 1 \mathrm{H}), 7.23-7.12(\mathrm{~m}, 1 \mathrm{H}), 7.00$ (ddd, $J=8.5,7.2,1.3 \mathrm{~Hz}$, 1H). (Fig. S26); $\left.{ }^{13} \mathbf{C ~ N M R ~ ( 7 5 ~ M H z , ~} \mathrm{CDCl}_{3}\right) \delta 154.85,137.28,133.52,124.76,120.02$, 119.84. (Fig. S27)<smiles>Oc1ccccc1Cl</smiles>

2-Chlorophenol (2k) ${ }^{\mathbf{1}} \mathbf{H}$ NMR $\left(300 \mathrm{MHz}, \mathrm{CDCl}_{3}\right) \delta 7.31$ (d, $\left.J=8.0 \mathrm{~Hz}, 1 \mathrm{H}\right), 7.17$ (dd, $J=$ 10.9, $4.5 \mathrm{~Hz}, 1 \mathrm{H}), 7.01$ (d, $J=8.1 \mathrm{~Hz}, 1 \mathrm{H}), 6.86$ (dd, $J=10.9,4.4 \mathrm{~Hz}, 1 \mathrm{H}), 5.68$ (s, 1H). (Fig. 
S28); ${ }^{13}$ C NMR (75 MHz, $\left.\mathrm{CDCl}_{3}\right) \delta 151.12,129.02,128.15,121.25,119.83,116.25$. (Fig. S29)<smiles>O=[N+]([O-])c1cccc(O)c1</smiles>

3-Nitrophenol (2I) ${ }^{1} \mathbf{H}$ NMR (300 MHz, $\left.\mathrm{CDCl}_{3}\right) \delta 7.86-7.78(\mathrm{~m}, 1 \mathrm{H}), 7.75-7.69(\mathrm{~m}, 1 \mathrm{H})$, $7.42(\mathrm{t}, J=8.2 \mathrm{~Hz}, 1 \mathrm{H}), 7.24-7.16(\mathrm{~m}, 1 \mathrm{H}), 5.66$ (s, 1H). (Fig. S30); ${ }^{13} \mathrm{C}$ NMR $(75 \mathrm{MHz}$, $\left.\mathrm{CDCl}_{3}\right) \delta 157.81,148.82,129.69,122.05,114.16,110.21$. (Fig. S31)<smiles>N#Cc1cccc(O)c1</smiles>

3-Hydroxybenzonitrile (2m) ${ }^{\mathbf{1}} \mathbf{H}$ NMR $\left(300 \mathrm{MHz}, \mathrm{CDCl}_{3}\right) \delta 7.86(\mathrm{dd}, J=4.0,2.6 \mathrm{~Hz}, 1 \mathrm{H})$, $7.83-7.69(\mathrm{~m}, 2 \mathrm{H}), 7.62$ (q, $J=7.4 \mathrm{~Hz}, 1 \mathrm{H})$. (Fig. S32); ${ }^{13} \mathbf{C}$ NMR $\left(75 \mathrm{MHz}, \mathrm{CDCl}_{3}\right) \delta$ $140.09,131.77,131.43,130.60,130.02,118.27,113.33$. (Fig. S33).<smiles>CC(C)c1ccc(O)cc1</smiles>

4-Isopropylphenol (2n) ${ }^{1} \mathbf{H}$ NMR $\left(300 \mathrm{MHz}, \mathrm{CDCl}_{3}\right) \delta 7.11(\mathrm{t}, J=8.0 \mathrm{~Hz}, 2 \mathrm{H}), 6.78(\mathrm{t}, J=$ $8.2 \mathrm{~Hz}, 2 \mathrm{H}), 5.01(\mathrm{~s}, 1 \mathrm{H}), 2.97-2.74(\mathrm{~m}, 1 \mathrm{H}), 1.21(\mathrm{~d}, J=6.9 \mathrm{~Hz}, 6 \mathrm{H})$. (Fig. S34); ${ }^{13} \mathrm{C}$ NMR $\left(75 \mathrm{MHz}, \mathrm{CDCl}_{3}\right) \delta 153.10,141.45,127.60,115.37,33.35,24.26$. (Fig. S35)<smiles>Oc1ccc(-c2ccccc2)cc1</smiles>

1,1'-Biphenyl]-4-ol (2o) ${ }^{1}$ H NMR (300 MHz, DMSO) $\delta 6.48(\mathrm{~s}, 1 \mathrm{H}), 4.99-4.92(\mathrm{~m}, 2 \mathrm{H})$, $4.90-4.78(\mathrm{~m}, 4 \mathrm{H}), 4.74-4.66(\mathrm{~m}, 1 \mathrm{H}), 4.36$ (dt, $J=4.7,2.7 \mathrm{~Hz}, 2 \mathrm{H})$. (Fig. S36); ${ }^{13} \mathbf{C ~ N M R}$ (75 MHz, DMSO) $\delta 157.18,140.95,132.19,128.92,128.16,126.54,116.15$. (Fig. S37).<smiles>Oc1ccc2ccccc2c1</smiles>

Naphthalen-2-ol (2p) ${ }^{1} \mathbf{H}$ NMR $\left(300 \mathrm{MHz}, \mathrm{CDCl}_{3}\right) \delta 8.18(\mathrm{dd}, J=6.7,3.0 \mathrm{~Hz}, 1 \mathrm{H}), 7.90-$ $7.70(\mathrm{~m}, 1 \mathrm{H}), 7.53-7.44(\mathrm{~m}, 2 \mathrm{H}), 7.30(\mathrm{dd}, J=10.5,5.2 \mathrm{~Hz}, 1 \mathrm{H}), 6.81(\mathrm{dd}, J=7.4,0.8 \mathrm{~Hz}$, 1H), 5.35 (s, 1H). (Fig. S38); ${ }^{13} \mathbf{C}$ NMR $\left(75 \mathrm{MHz}, \mathrm{CDCl}_{3}\right) \delta 151.10,134.67,127.65,126.40$, 125.80, 125.28, 124.29, 121.41, 120.72, 108.76. (Fig. S39)<smiles>Oc1ccc(Oc2ccccc2)cc1</smiles> 
4-Phenoxyphenol (2q) ${ }^{1} \mathbf{H}$ NMR $\left(300 \mathrm{MHz}, \mathrm{CDCl}_{3}\right) \delta 7.38-7.20(\mathrm{~m}, 2 \mathrm{H}), 7.04(\mathrm{t}, J=6.9$ $\mathrm{Hz}, 1 \mathrm{H}), 6.98-6.87$ (m, 3H), $6.87-6.73$ (m, 2H), 4.82 (s, 1H). (Fig. S40); ${ }^{13}$ C NMR (75 $\left.\mathrm{MHz}, \mathrm{CDCl}_{3}\right) \delta 158.36,151.65,150.29,129.77,122.72,121.09,117.75,116.53$. (Fig. S41)<smiles>Oc1ccncc1</smiles>

Pyridin-4-ol (2r) ${ }^{1} \mathbf{H}$ NMR (400 MHz, MeOD) $\delta 7.82(\mathrm{~d}, J=7.5 \mathrm{~Hz}, 2 \mathrm{H}), 6.50(\mathrm{dd}, J=7.9$, $2.1 \mathrm{~Hz}, 2 \mathrm{H}), 4.94$ (s, 1H). (Fig. S42); ${ }^{13} \mathrm{C}$ NMR (101 MHz, MeOD) $\delta$ 181.73, 139.99, 117.94. (Fig. S43)<smiles>Oc1cccnc1</smiles>

Pyridin-3-ol (2s) ${ }^{\mathbf{1}} \mathbf{H}$ NMR (400 MHz, MeOD) $\delta 8.10(\mathrm{dd}, J=2.4,1.1 \mathrm{~Hz}, 1 \mathrm{H}), 8.01(\mathrm{dd}, J=$ 4.1, $2.0 \mathrm{~Hz}, 1 \mathrm{H}), 7.26$ (dt, $J=4.4,1.5 \mathrm{~Hz}, 2 \mathrm{H}), 4.98$ (s, 1H). (Fig. S44); ${ }^{13}$ C NMR $(101 \mathrm{MHz}$, MeOD) $\delta 145.95,130.85,128.32,115.85,114.47$. (Fig. S45)<smiles>Oc1cn[nH]c1</smiles>

4-Hydroxypyrazole (2t) ${ }^{1}$ H NMR (400 MHz, MeOD) $\delta 7.67$ (s, 1H), 5.16 (s, 2H). (Fig. S46); ${ }^{13}$ C NMR (101 MHz, MeOD) $\delta$ 143.90, 122. (Fig. S47)<smiles>Oc1ccc(-c2nc3ccccc3[nH]2)cc1</smiles>

\section{4-(1H-benzo $[d]$ imidazol-2-yl)phenol (2u)}

${ }^{1}$ H NMR (300 MHz, DMSO) $\delta: 9.99(\mathrm{~s}, 1 \mathrm{H}), 8.00(\mathrm{~d}, J=8.6 \mathrm{~Hz}, 2 \mathrm{H}), 7.53(\mathrm{dd}, J=5.9,3.2$ $\mathrm{Hz}, 2 \mathrm{H}), 7.15$ (dd, $J=6.0,3.2 \mathrm{~Hz}, 2 \mathrm{H}), 6.91(\mathrm{~d}, J=8.6 \mathrm{~Hz}, 2 \mathrm{H}), 6.55$ (s, 1H). (Fig. S48) ${ }^{\mathbf{1 3}} \mathbf{C}$ NMR (75 MHz, DMSO) $\delta: 163.19,159.28,151.84,139.28,128.28,121.77,121.05,115.79$. (Fig. S49)<smiles>Oc1ccc(O)cc1</smiles>

Hydroquinone (2v) ${ }^{1} \mathbf{H}$ NMR $\left(300 \mathrm{MHz}, \mathrm{CDCl}_{3}\right) \delta 8.18(\mathrm{~d}, J=5.8 \mathrm{~Hz}, 2 \mathrm{H}), 6.76-6.56(\mathrm{~m}$, 4H). (Fig. S50); ${ }^{13} \mathbf{C}$ NMR $\left(75 \mathrm{MHz}, \mathrm{CDCl}_{3}\right) \delta 148.99,115.10$. (Fig. S51) 
<smiles>Oc1cc(-c2ccccc2)cc(-c2ccccc2)c1</smiles>

[1,1':3',1"-terphenyl]-5'-ol (2w) ${ }^{\mathbf{1}} \mathbf{H}$ NMR (300 MHz, $\left.\mathrm{CDCl}_{3}\right) \delta 7.62-7.55(\mathrm{~m}, 4 \mathrm{H}), 7.50-$ $7.29(\mathrm{~m}, 8 \mathrm{H}), 7.04(\mathrm{~d}, J=1.5 \mathrm{~Hz}, 2 \mathrm{H}), 5.62(\mathrm{~s}, 1 \mathrm{H})$. (Fig. S52) ${ }^{\mathbf{1 3}} \mathbf{C} \mathbf{N M R}\left(75 \mathrm{MHz}, \mathrm{CDCl}_{3}\right) \delta$ 156.23,143.52, 140.78, 142.08, 127.70, 127.57, 127.04, 118.90, 113.10. (Fig. S53)<smiles>CCOC(=O)C1=C(C)NC(C)=C(C(=O)OCC)C1c1ccc(O)cc1</smiles>

Diethyl4-(4-hydroxyphenyl)-2,6-dimethyl-1,4 dihydropyridine-3,5-dicarboxylate (2x) ${ }^{1}$ H NMR (300 MHz, DMSO) $\delta 9.09(\mathrm{~s}, 1 \mathrm{H}), 8.72(\mathrm{~s}, 1 \mathrm{H}), 6.92(\mathrm{~d}, J=8.5 \mathrm{~Hz}, 2 \mathrm{H}), 6.57(\mathrm{~d}, J$ $=8.4 \mathrm{~Hz}, 2 \mathrm{H}), 4.74(\mathrm{~s}, 1 \mathrm{H}), 4.02-3.93(\mathrm{~m}, 4 \mathrm{H}), 2.23(\mathrm{~s}, 6 \mathrm{H}), 1.13(\mathrm{t}, J=7.1 \mathrm{~Hz}, 6 \mathrm{H})$. (Fig. S54); ${ }^{13}$ C NMR (75 MHz, DMSO) $\delta 167.53,155.85,145.19,139.34,128.70,114.94,102.72$, 59.31, 18.62, 14.62. (Fig. S55)<smiles>O=Cc1ccc(O)cc1</smiles>

4-Hydroxybenzaldehyde (2y) ${ }^{\mathbf{1}} \mathbf{H}$ NMR $\left(300 \mathrm{MHz}, \mathrm{CDCl}_{3}\right) \delta 9.85(\mathrm{~s}, 1 \mathrm{H}), 7.83(\mathrm{~d}, J=8.6 \mathrm{~Hz}$, 2H), 7.21 (s, 1H), 7.00 (d, $J=8.5 \mathrm{~Hz}, 2 \mathrm{H})$. (Fig. S56) ${ }^{13} \mathbf{C}$ NMR (75 MHz, $\left.\mathrm{CDCl}_{3}\right) \delta 190.02$, 162.82, 131.41, 127.83,115.28. (Fig. S57)<smiles>OC1CCCCC1</smiles>

Cyclohexanol (2z) ${ }^{1} \mathbf{H}$ NMR $\left(300 \mathrm{MHz}, \mathrm{CDCl}_{3}\right) \delta 3.82-3.51(\mathrm{~m}, 1 \mathrm{H}), 1.92(\mathrm{dd}, J=10.0$, $4.6 \mathrm{~Hz}, 3 \mathrm{H}), 1.74(\mathrm{~d}, J=8.8 \mathrm{~Hz}, 2 \mathrm{H}), 1.55(\mathrm{~d}, J=10.9 \mathrm{~Hz}, 1 \mathrm{H}), 1.40-1.12$ (m, 5H). (Fig. S58); ${ }^{13}$ C NMR (75 MHz, $\left.\mathrm{CDCl}_{3}\right)$ 69.70, 35.11, 25.23, 24.00. (Fig. S59). 


\section{References}

S1. Hummers W. S.; Offeman, R. E. Preparation of graphitic oxide. J. Am. Chem. Soc., 1958, 80, 1339, DOI: 10.1021/ja01539a017.

S2. Paredes, J. I.; Rodil, S. V.; Alonso A. M.; Tascón, J. M. D. Graphene oxide dispersion in organic solvents. Langmuir, 2008, 24, 10560-10564, DOI: 10.1021/la801744a.

S3. Steglich W.; Neises, B. Simple method for the esterification of carboxylic acids. Angew. Chem. Int. Ed. I978, 17, 522-524, DOI 10.1002/anie.197805221.

S4. Dreyer, D. R.; Jia, H. P.; Bielawski, C. W. Graphene oxide: a convenient carbocatalyst for facilitating oxidation and hydration reactions. Angew. Chem. Int. Ed. 2010, 49, 6813-6816, DOI 10.1002/anie.201002160.

S5. Jia, H. P.; Dreyer D. R.; Bielawski, C.W. C-H Oxidation using graphite oxide. Tetrahedron, 2011, 67, 4431-4434, DOI 10.1016/j.tet.2011.02.065.

S6. Huang, H.; Huang, J.; Liu, Y.-M.; He, H.-Y.; Cao, Y. Fan, K.-N. Graphite oxide as an efficient and durable metal-free catalyst for aerobic oxidative coupling of amines to imines. Green Chem. 2012, 14, 930-934, DOI 10.1039/C2GC16681j.

S7. Chu, X.; Zhu, Q.; Dai W.-L.; Fan, K. Excellent Catalytic Performance of Graphite oxide in the Selective Oxidation of Glutaraldehyde by Aqueous Hydrogen peroxide. RSC Advances, 2012, 2, 7135-7139, DOI 10.1039/C2RA21068a.

S8. Gonçalves, G. A. B.; Pires, S. M. G.; Simoes, M. M. Q.; Nevesb M. G. P. M. S.; Marques, P. A. A. P. Three-dimensional graphene oxide: a promising green and sustainable catalyst for oxidation reactions at room temperature. Chem. Commun. 2014, 50, 7673-7676, DOI 10.1039/C4CC02092h.

S9.Lv, G.; Wang, H.; Yang, Y.; Deng, T.; Chen, C.; Zhu, Y.; Hou, X. Graphene oxide: a convenient metal-free carbocatalyst for facilitating aerobic oxidation of 5hydroxymethylfurfural into 2,5- diformylfuran. ACS Catal. 2015, 5, 5636-5646, DOI 10.1021/acscatal.5b01446.

S10. Cui, Y.; Lee, Y. H.; Yang, J. W. Impact of carboxyl groups in graphene oxide on chemoselective alcohol oxidation with ultra-Low carbocatalyst loading. Sci. Rep. 2017, 7, 3146-3154, DOI 10.1038/s41598-017-03468-4. 


\section{NMR Spectra of ipso-Hydroxylated Products}

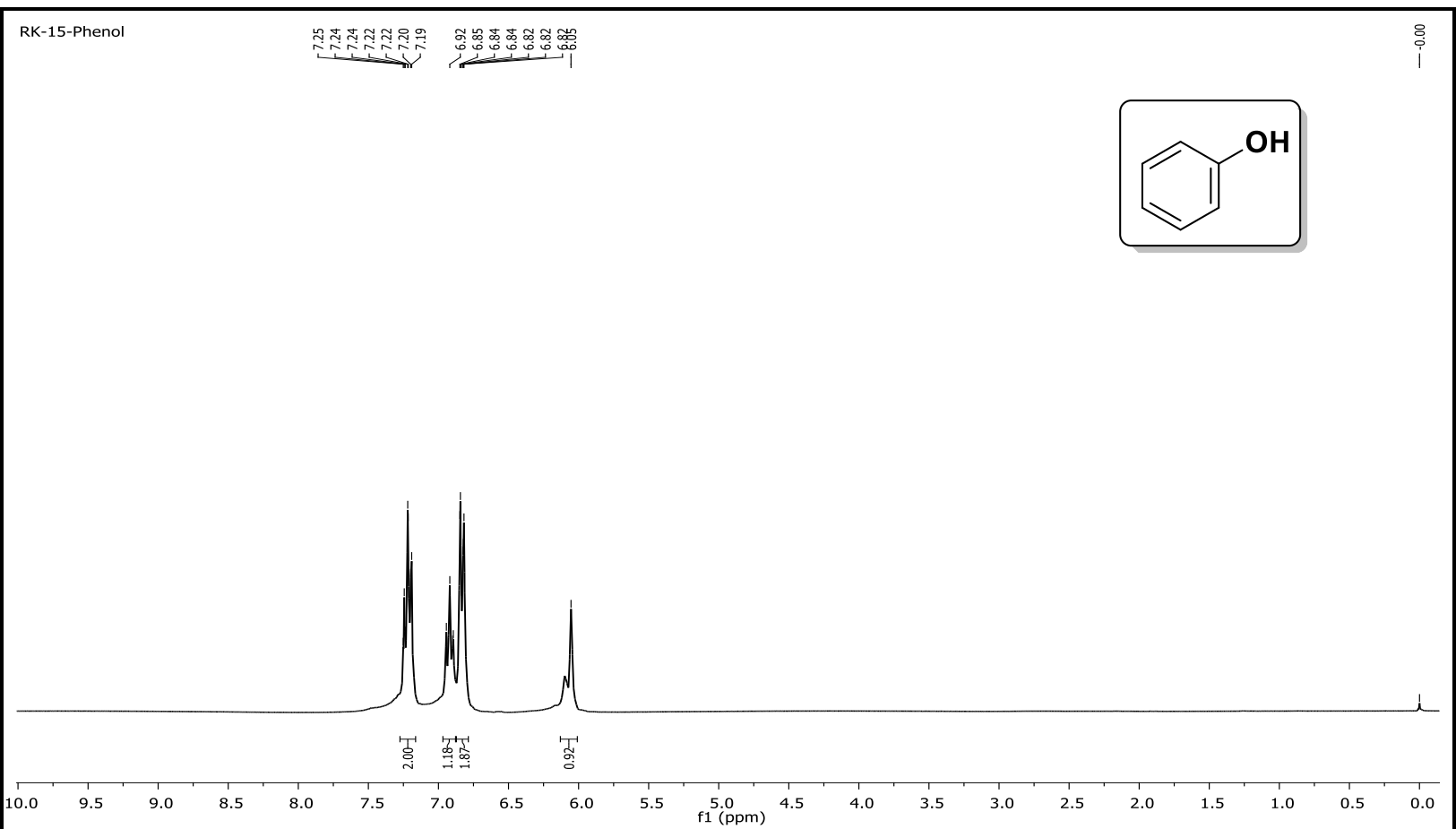

Fig. S8 ${ }^{1} \mathrm{H}$ NMR Spectrum of Phenol $2 \mathbf{a}\left(300 \mathrm{MHz}, \mathrm{CDCl}_{3}\right)$

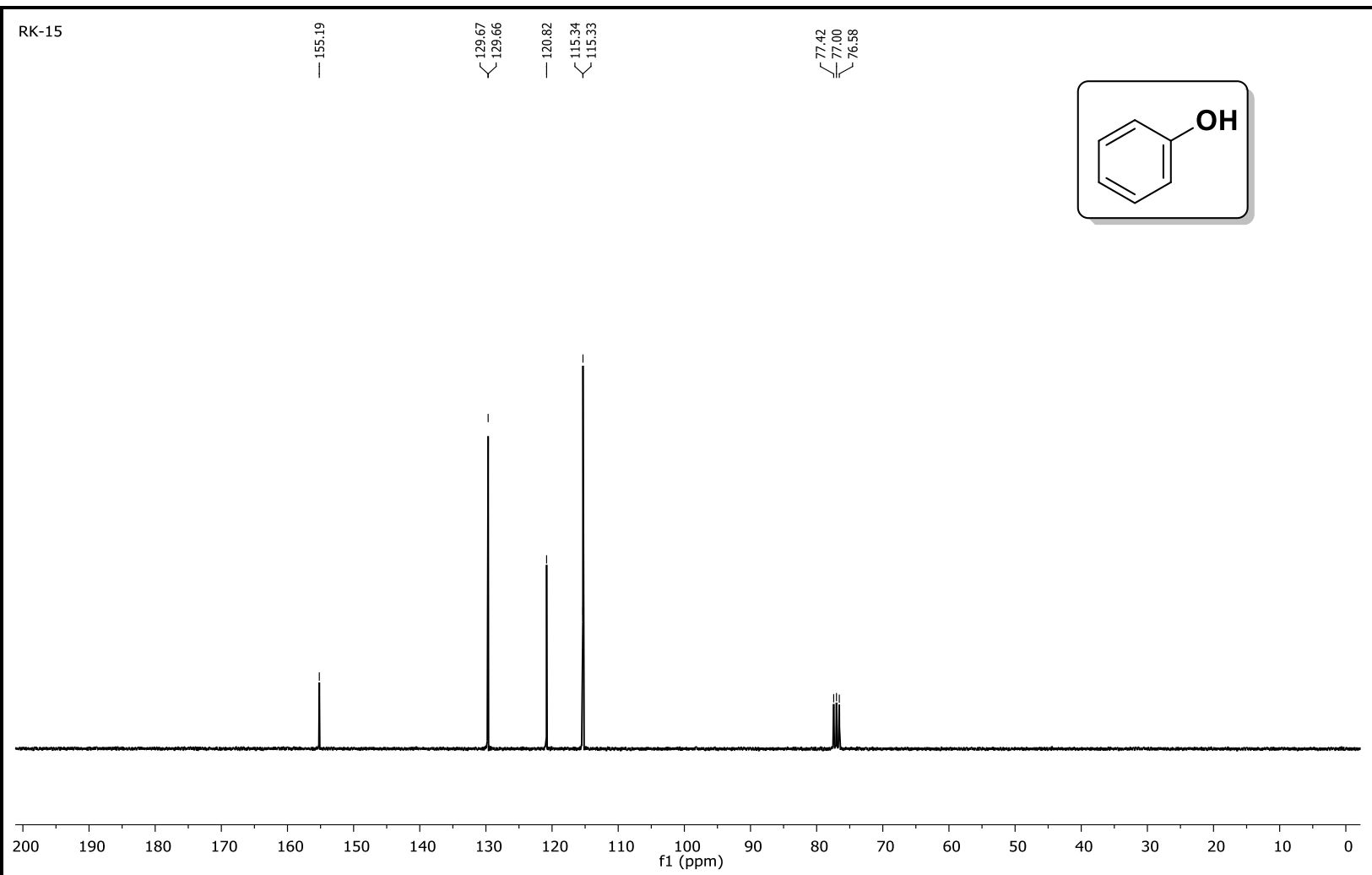

Fig. S9 ${ }^{13} \mathrm{C}$ NMR Spectrum of Phenol $2 \mathbf{a}\left(75 \mathrm{MHz}, \mathrm{CDCl}_{3}\right)$ 


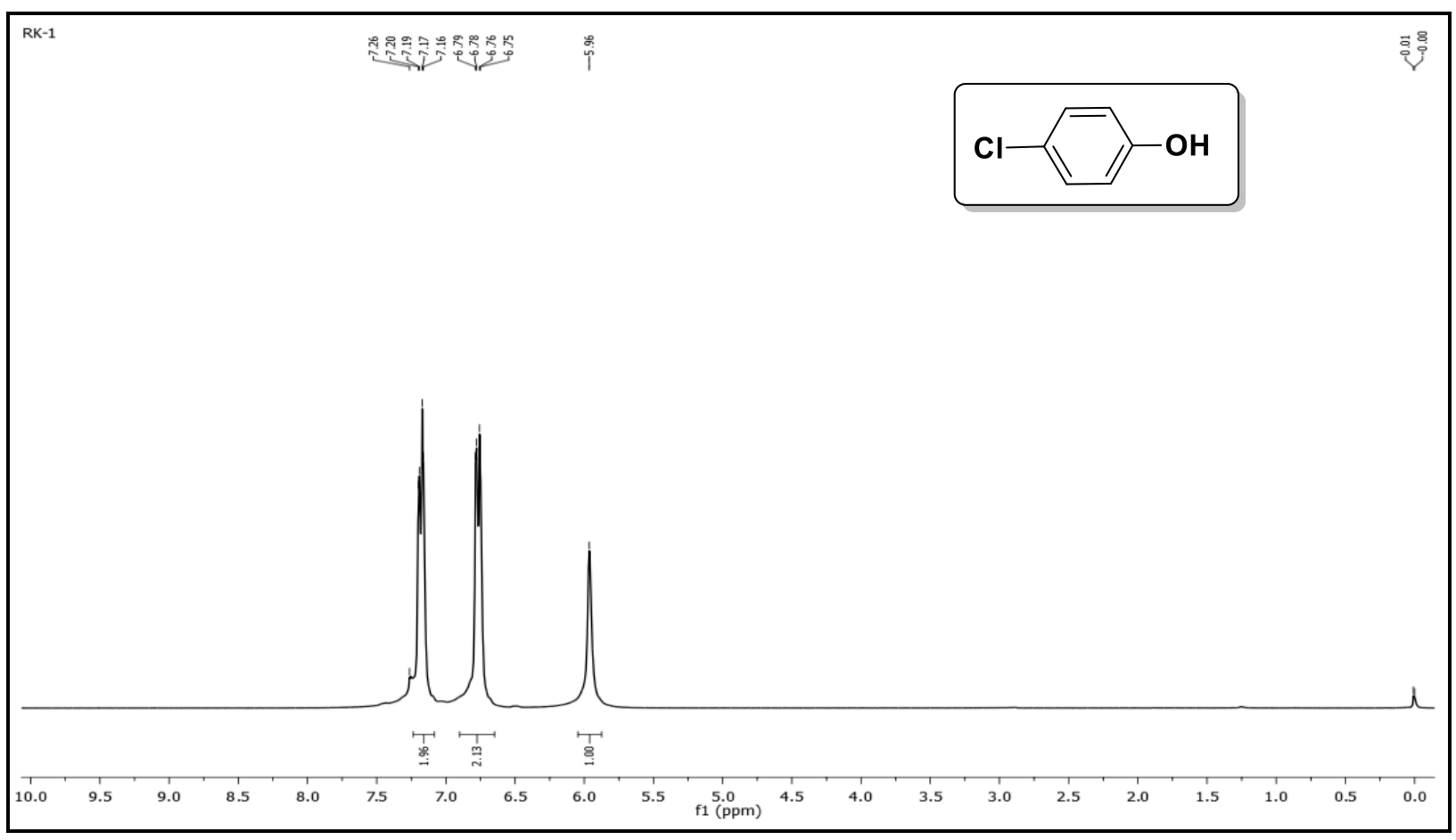

Fig. S10 ${ }^{1} \mathrm{H}$ NMR Spectrum of 4-chlorophenol (2b) (300 MHz, $\left.\mathrm{CDCl}_{3}\right)$

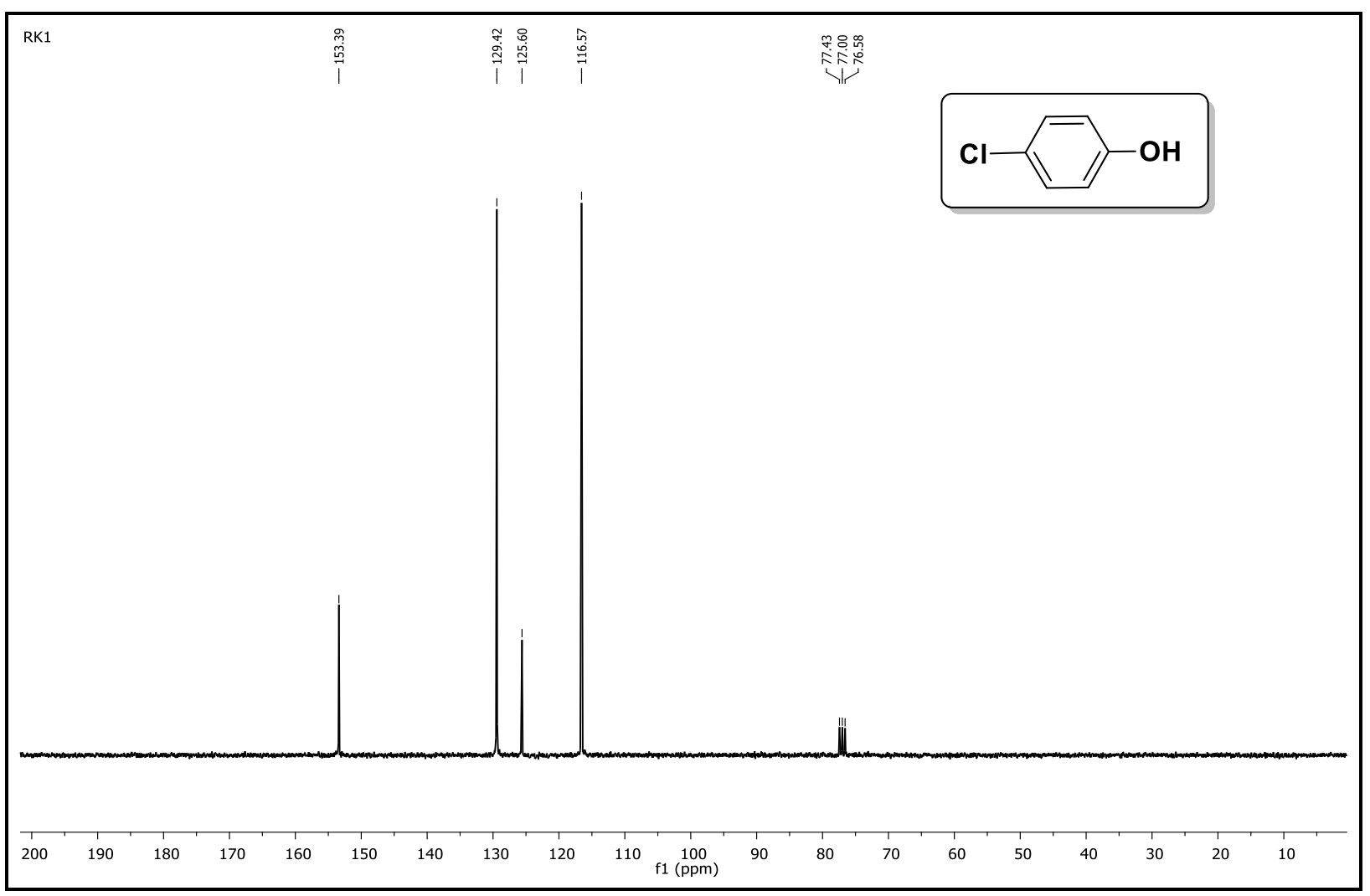

Fig. S11 ${ }^{13} \mathrm{C}$ NMR Spectrum of 4-chlorophenol (2b) (75 $\left.\mathrm{MHz}, \mathrm{CDCl}_{3}\right)$ 


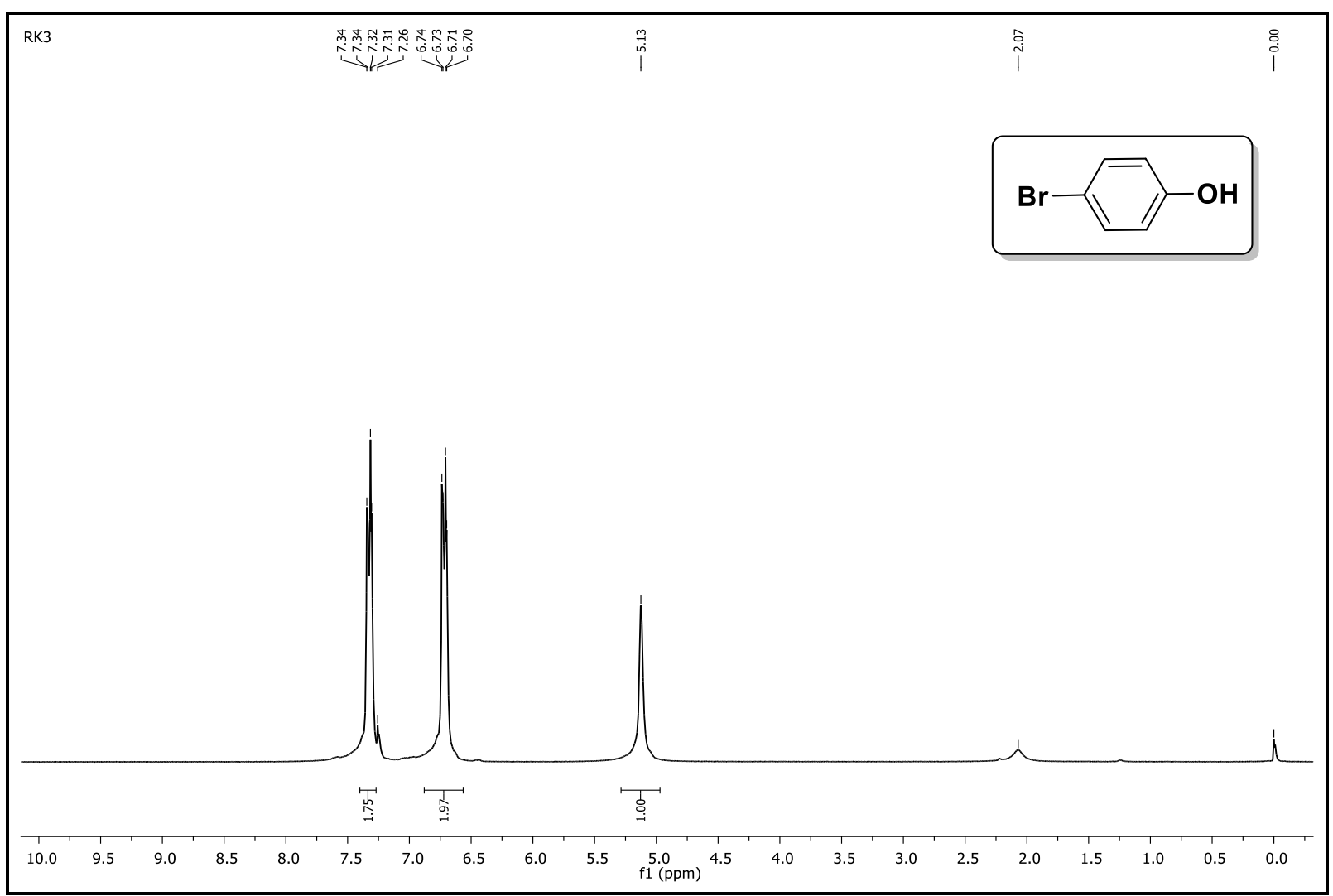

Fig. S12 ${ }^{1} \mathrm{H}$ NMR Spectrum of 4-bromophenol (2c) $\left(300 \mathrm{MHz}, \mathrm{CDCl}_{3}\right)$

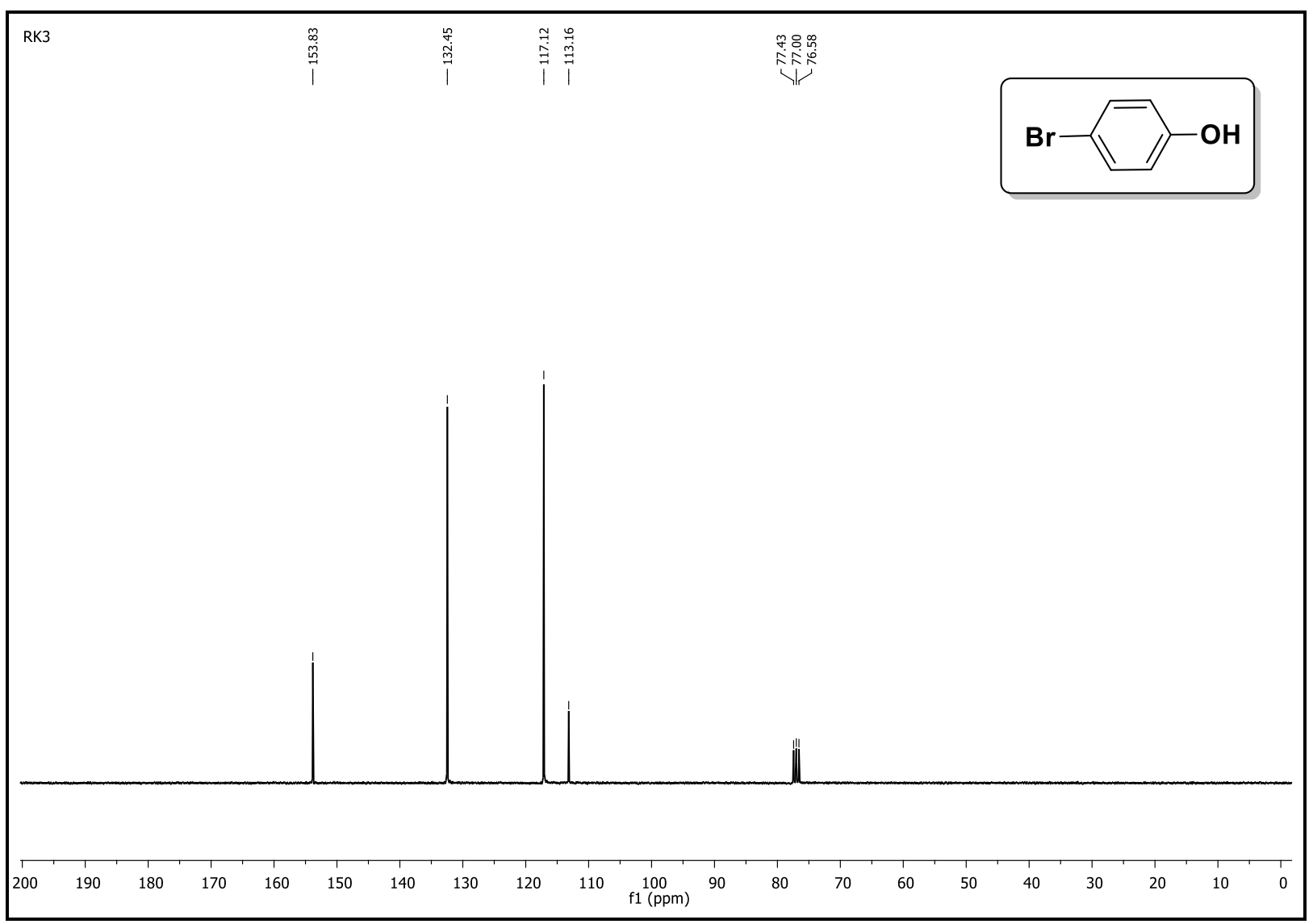

Fig. S13 ${ }^{13} \mathrm{C}$ NMR Spectrum of 4-bromophenol (2c) $\left(75 \mathrm{MHz}, \mathrm{CDCl}_{3}\right)$ 


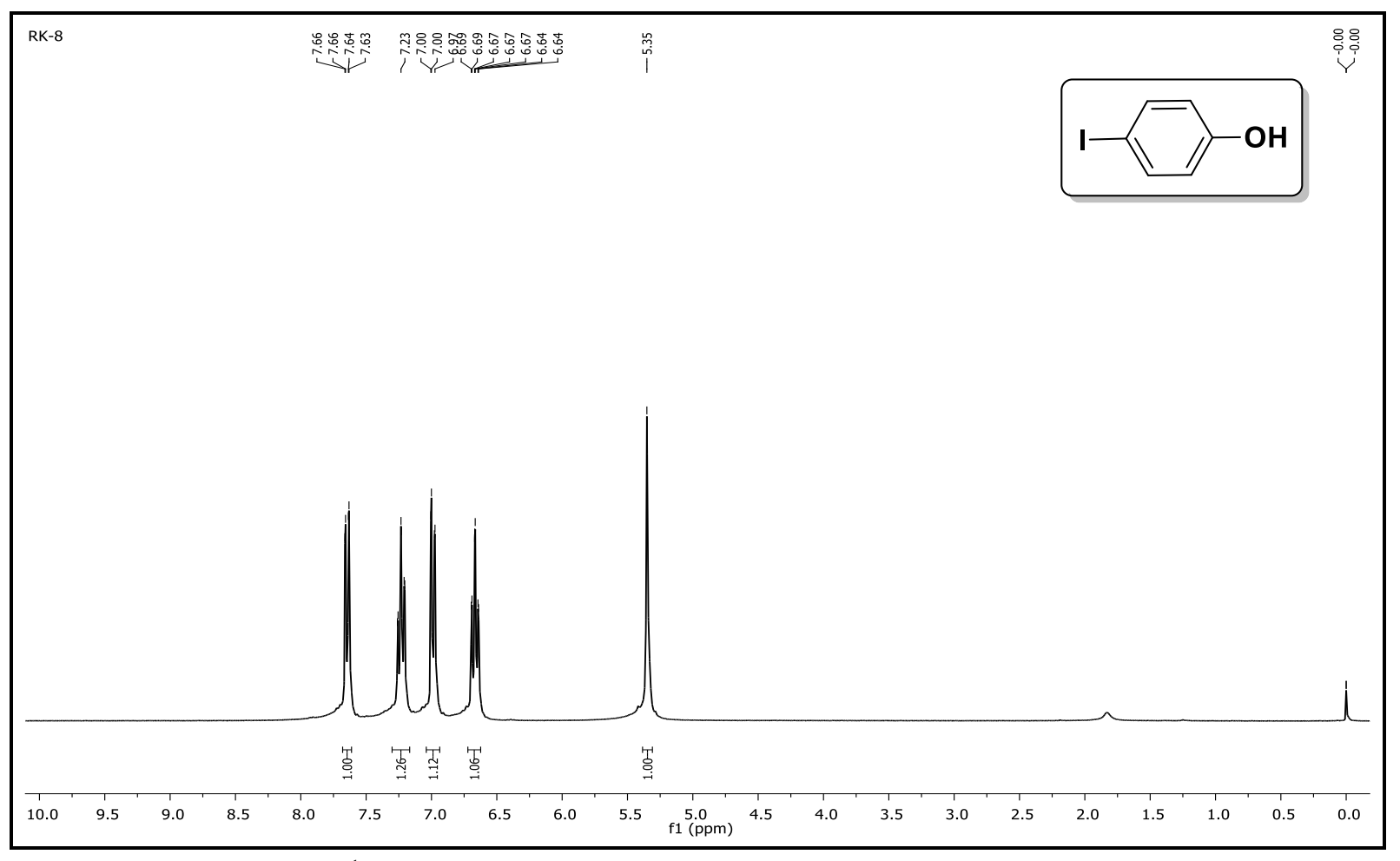

Fig. S14 ${ }^{1} \mathrm{H}$ NMR Spectrum of 4-iodophenol (2d) $\left(300 \mathrm{MHz}, \mathrm{CDCl}_{3}\right)$

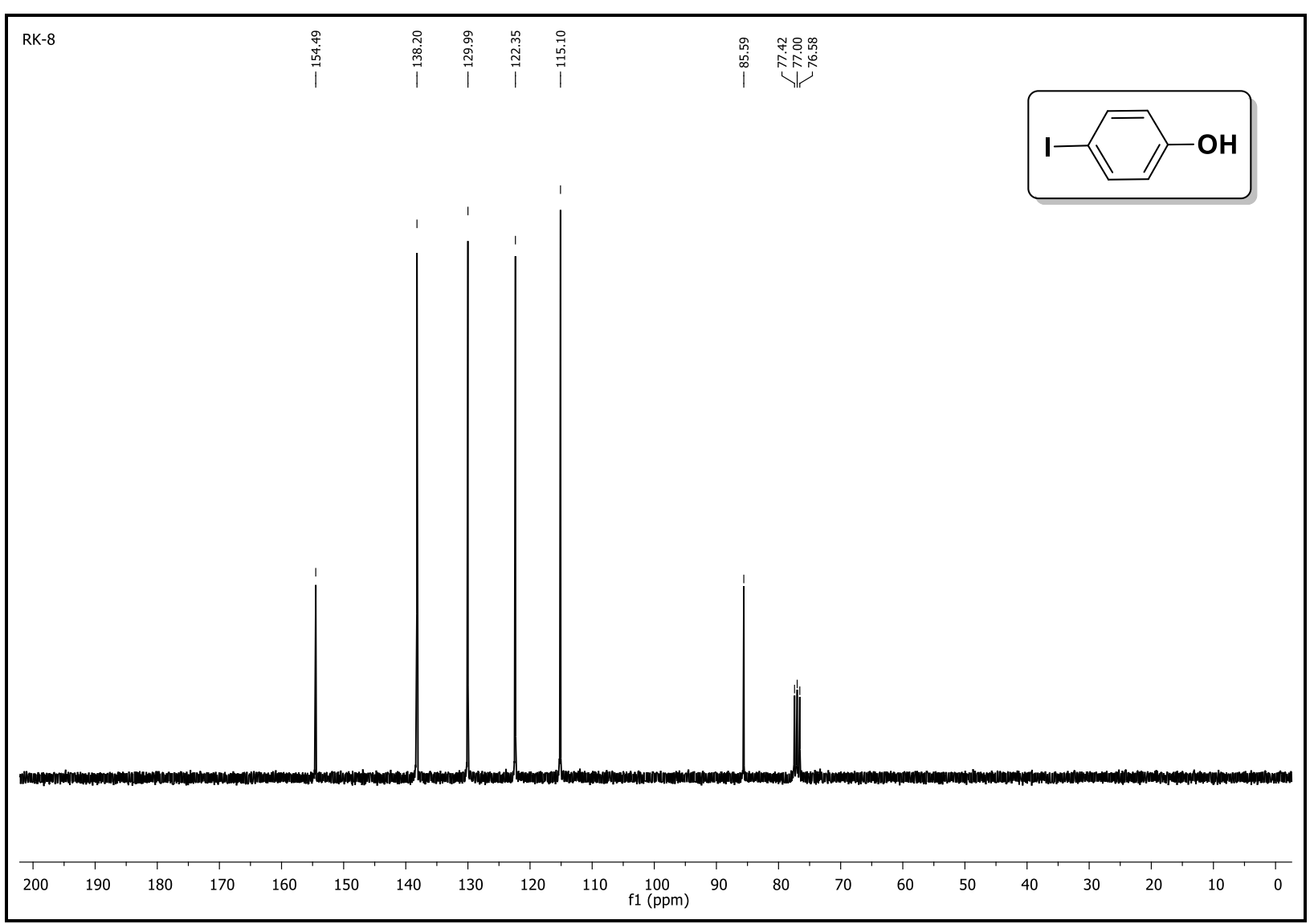

Fig. S15 ${ }^{13} \mathrm{C}$ NMR Spectrum of 4-iodophenol (2d) $\left(75 \mathrm{MHz}, \mathrm{CDCl}_{3}\right)$ 


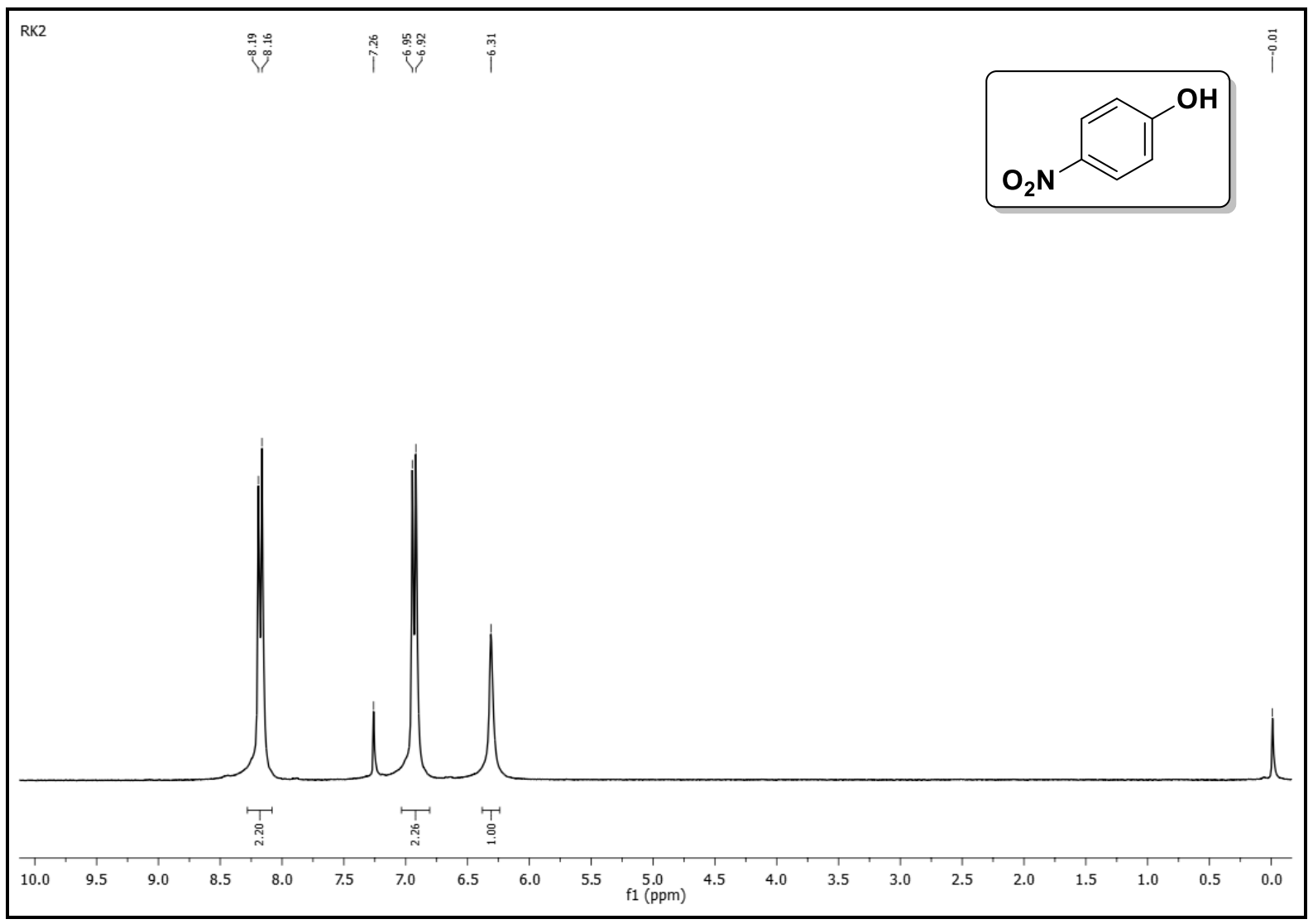

Fig. S16 ${ }^{1} \mathrm{H}$ NMR Spectrum of 4-Nitrophenol (2e) (300 MHz, $\left.\mathrm{CDCl}_{3}\right)$

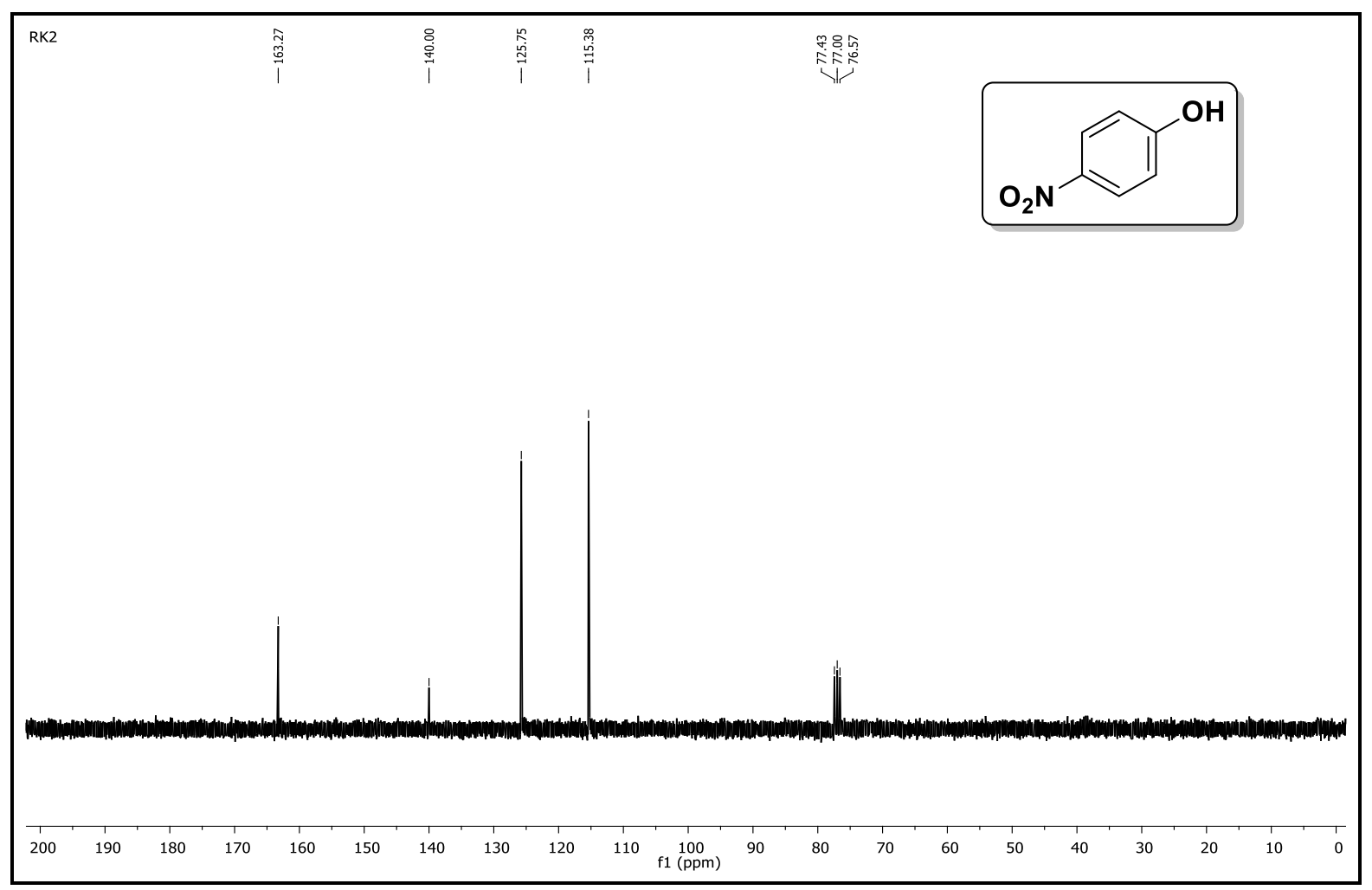

Fig. S17 ${ }^{13} \mathrm{C}$ NMR Spectrum of 4-Nitrophenol (2e) $\left(75 \mathrm{MHz}, \mathrm{CDCl}_{3}\right)$ 


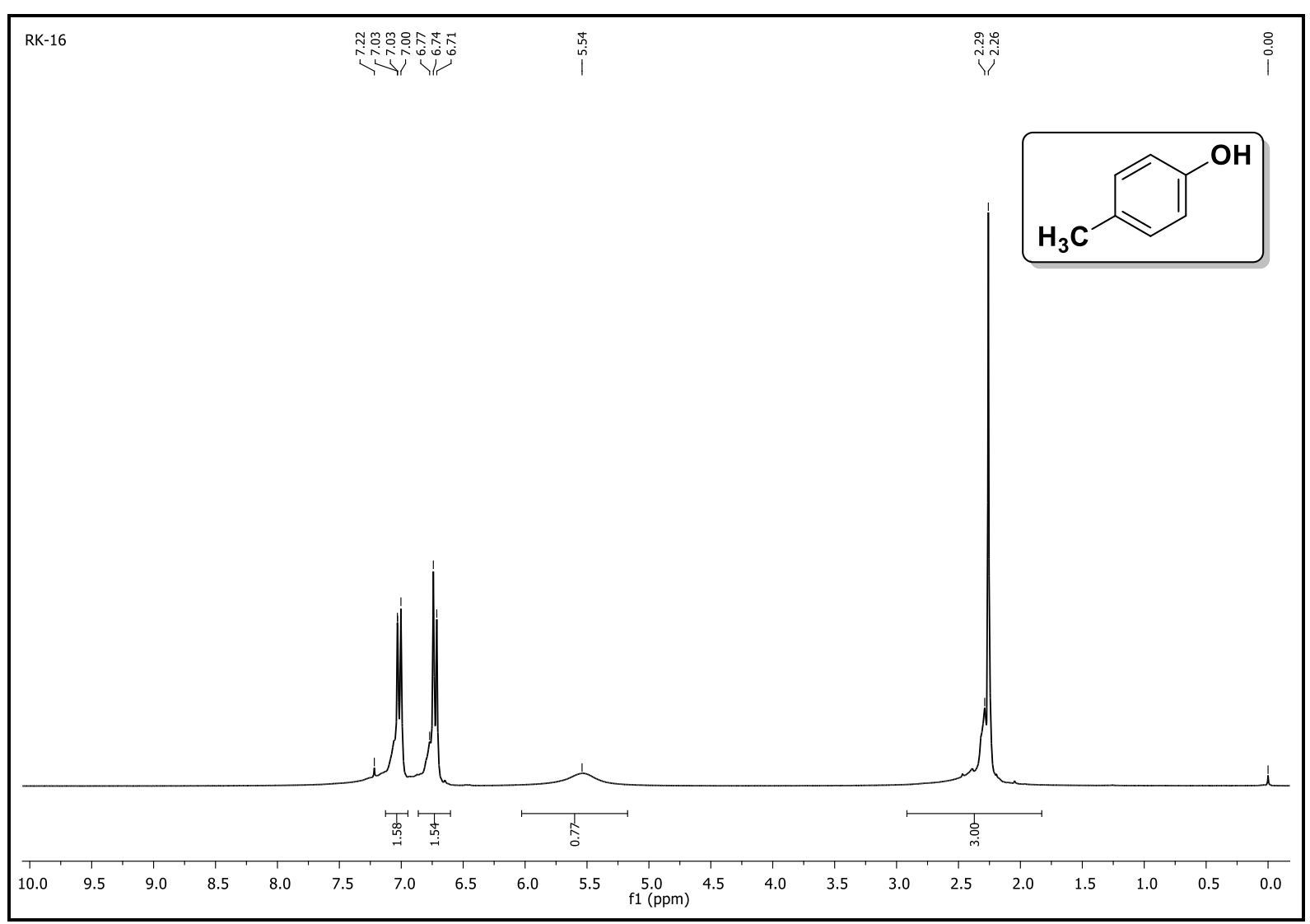

Fig. S18 ${ }^{1} \mathrm{H}$ NMR Spectrum of 4-methylphenol (2f) (300 MHz, $\left.\mathrm{CDCl}_{3}\right)$

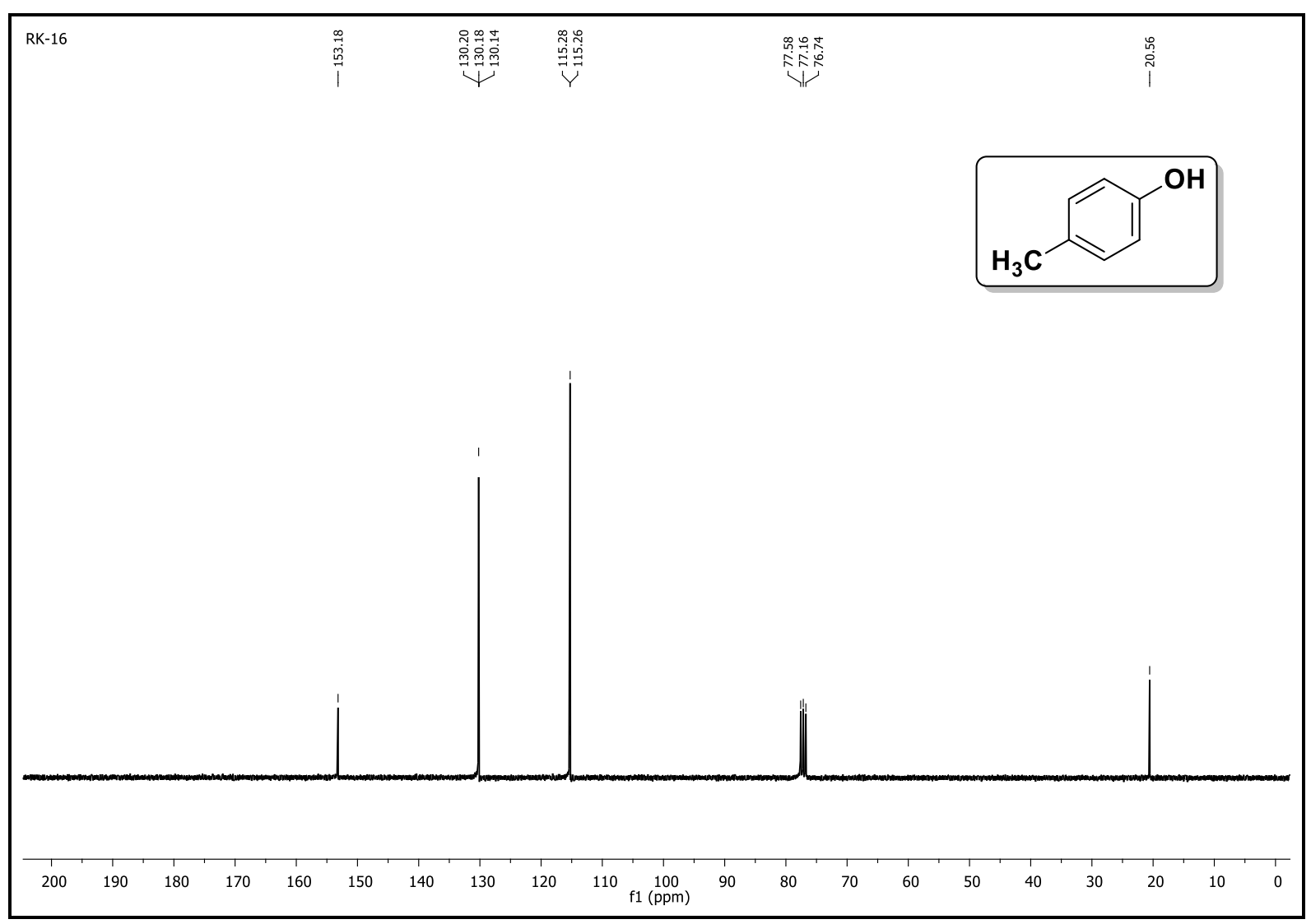

Fig. S19 ${ }^{13} \mathrm{C}$ NMR Spectrum of 4-methylphenol (2f) $\left(75 \mathrm{MHz}, \mathrm{CDCl}_{3}\right)$ 


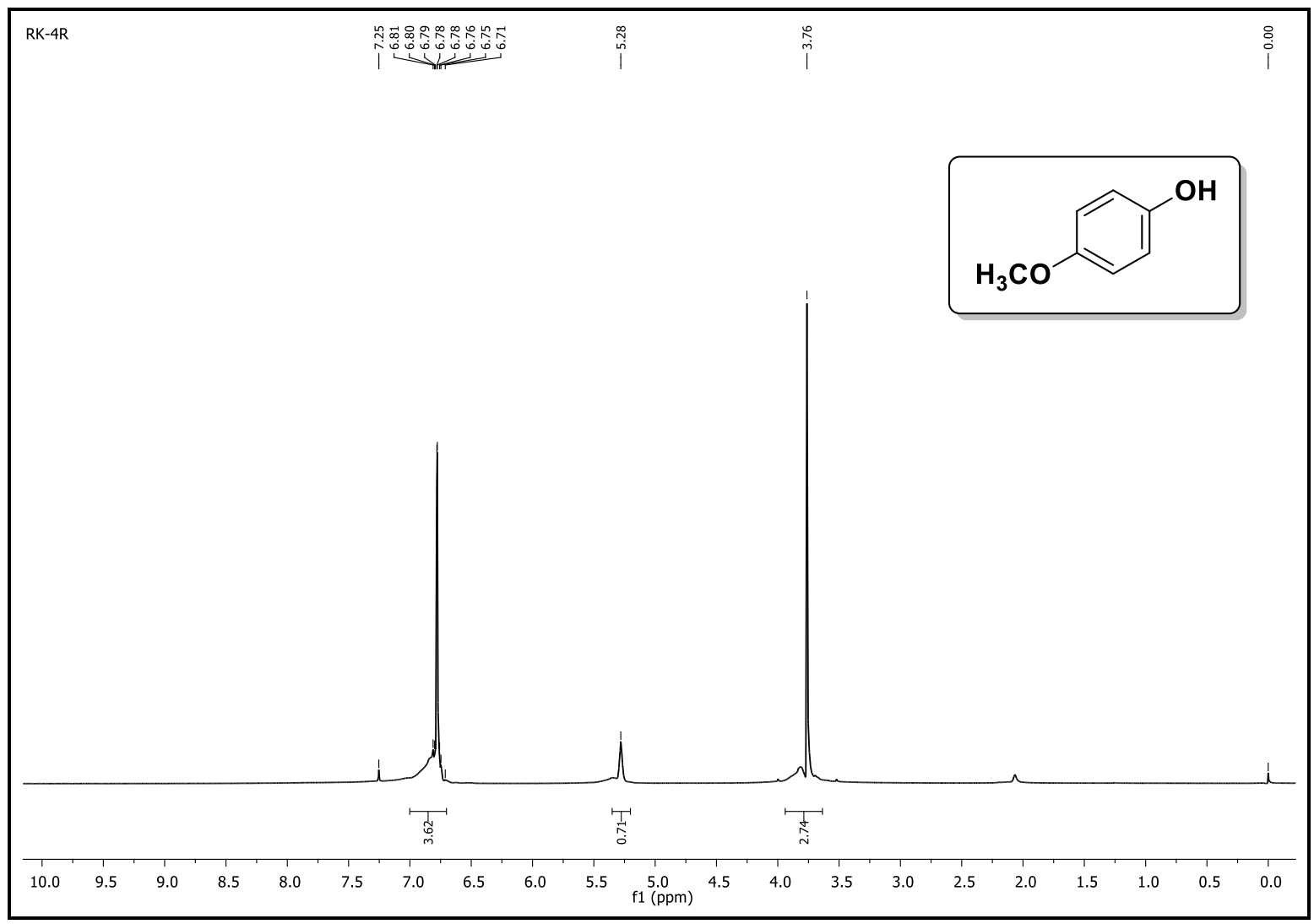

Fig. S20 ${ }^{1} \mathrm{H}$ NMR Spectrum of 4-methoxyphenol (2g) (300 MHz, $\mathrm{CDCl}_{3}$ )

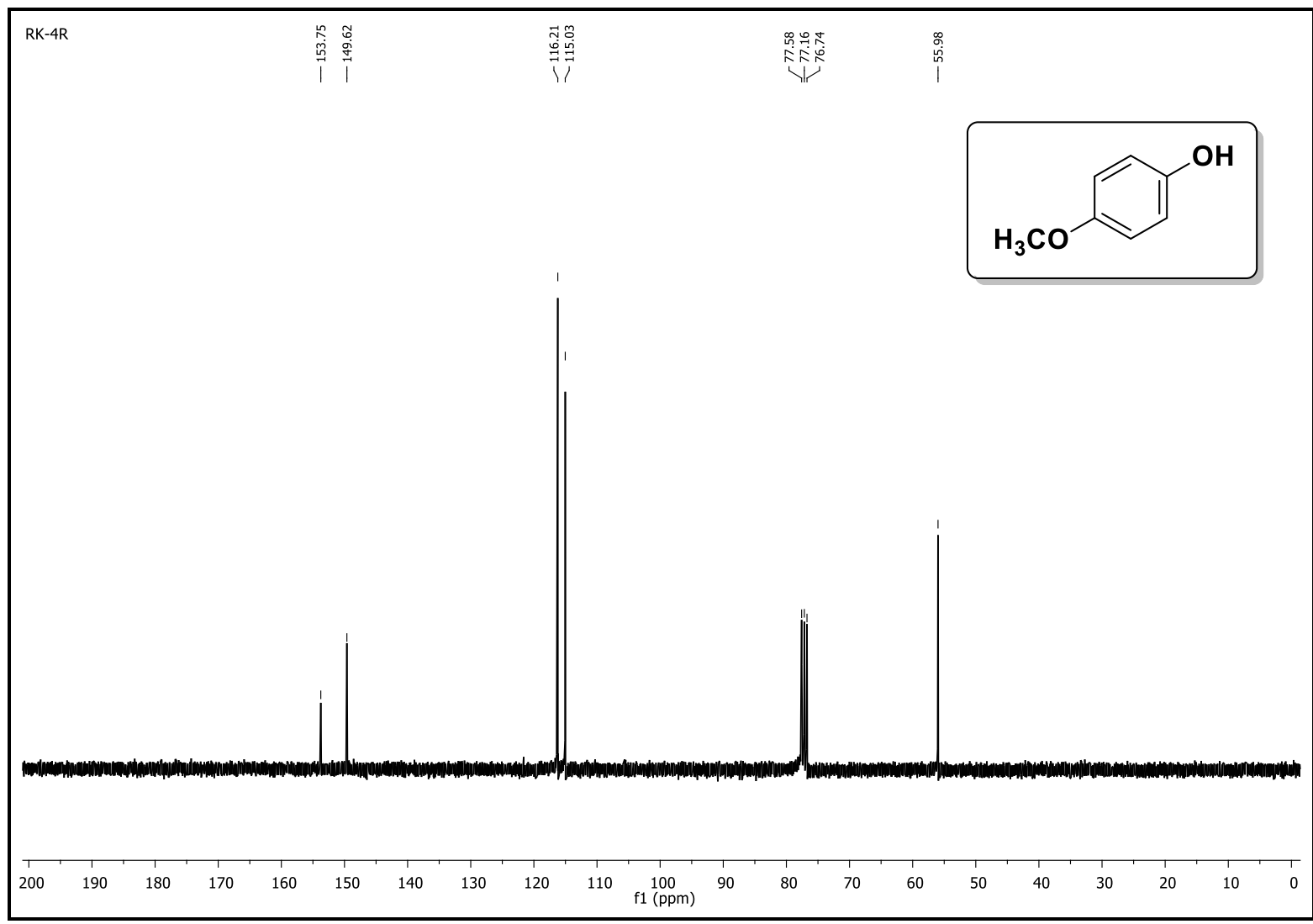

Fig. S21 ${ }^{13} \mathrm{C}$ NMR Spectrum of 4-methoxyphenol (2g) $\left(75 \mathrm{MHz}, \mathrm{CDCl}_{3}\right)$ 


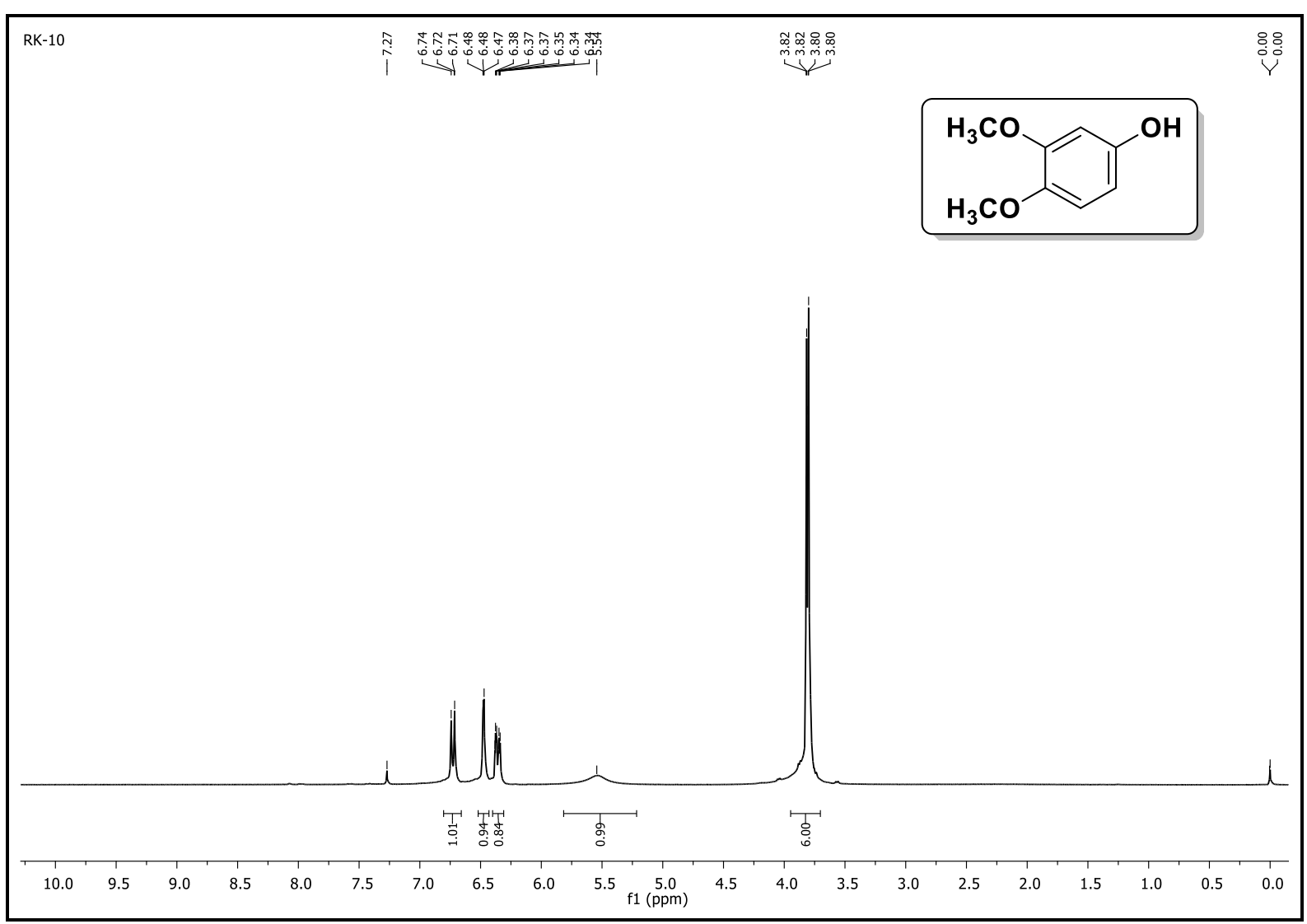

Fig. S22 ${ }^{1} \mathrm{H}$ NMR Spectrum of 3,4-dimethoxyphenol (2h) (300 MHz, $\left.\mathrm{CDCl}_{3}\right)$

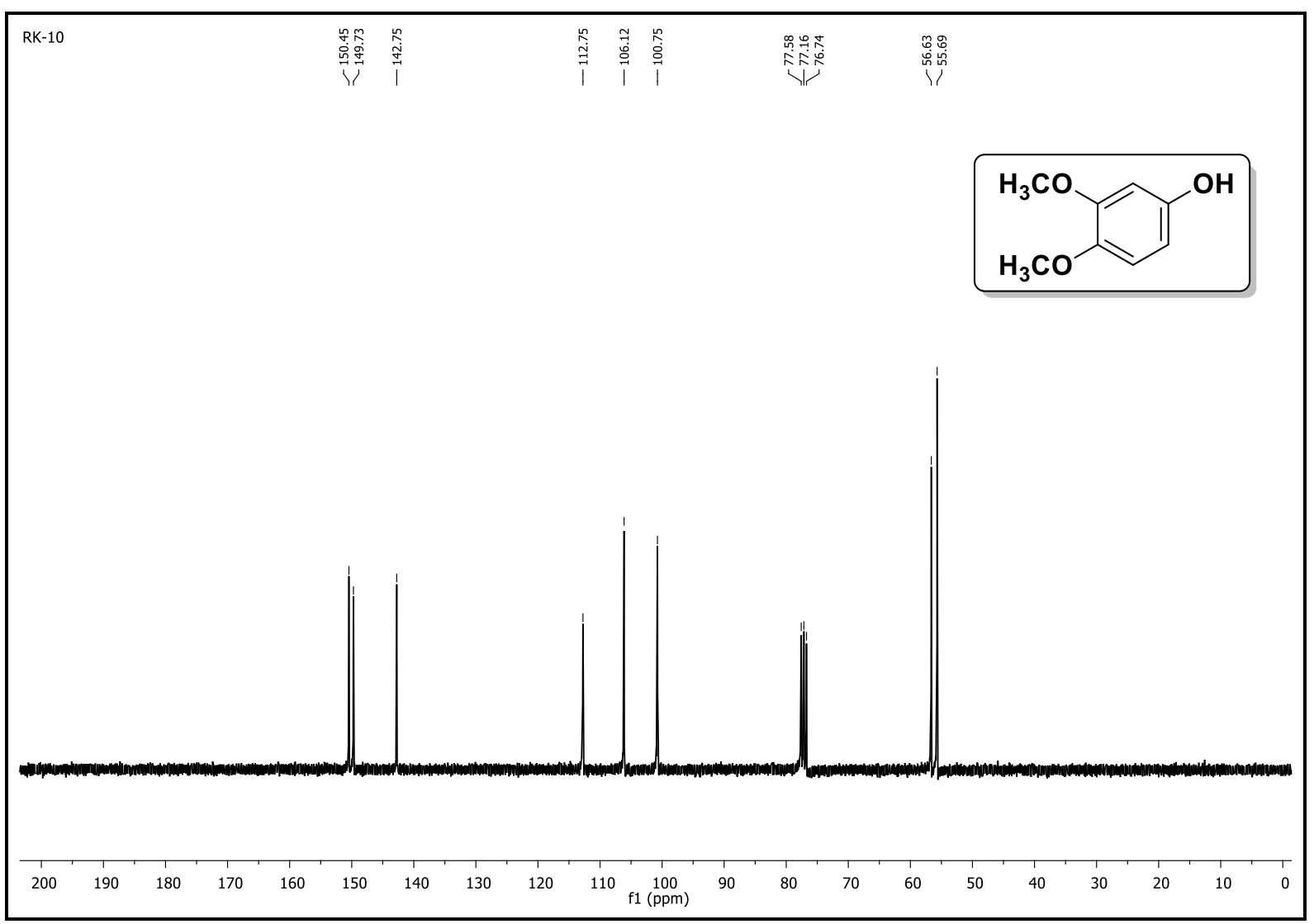

Fig. $\mathbf{S 2 3}{ }^{13} \mathrm{C}$ NMR Spectrum of 3,4-dimethoxyphenol (2h) $\left(75 \mathrm{MHz}, \mathrm{CDCl}_{3}\right)$ 


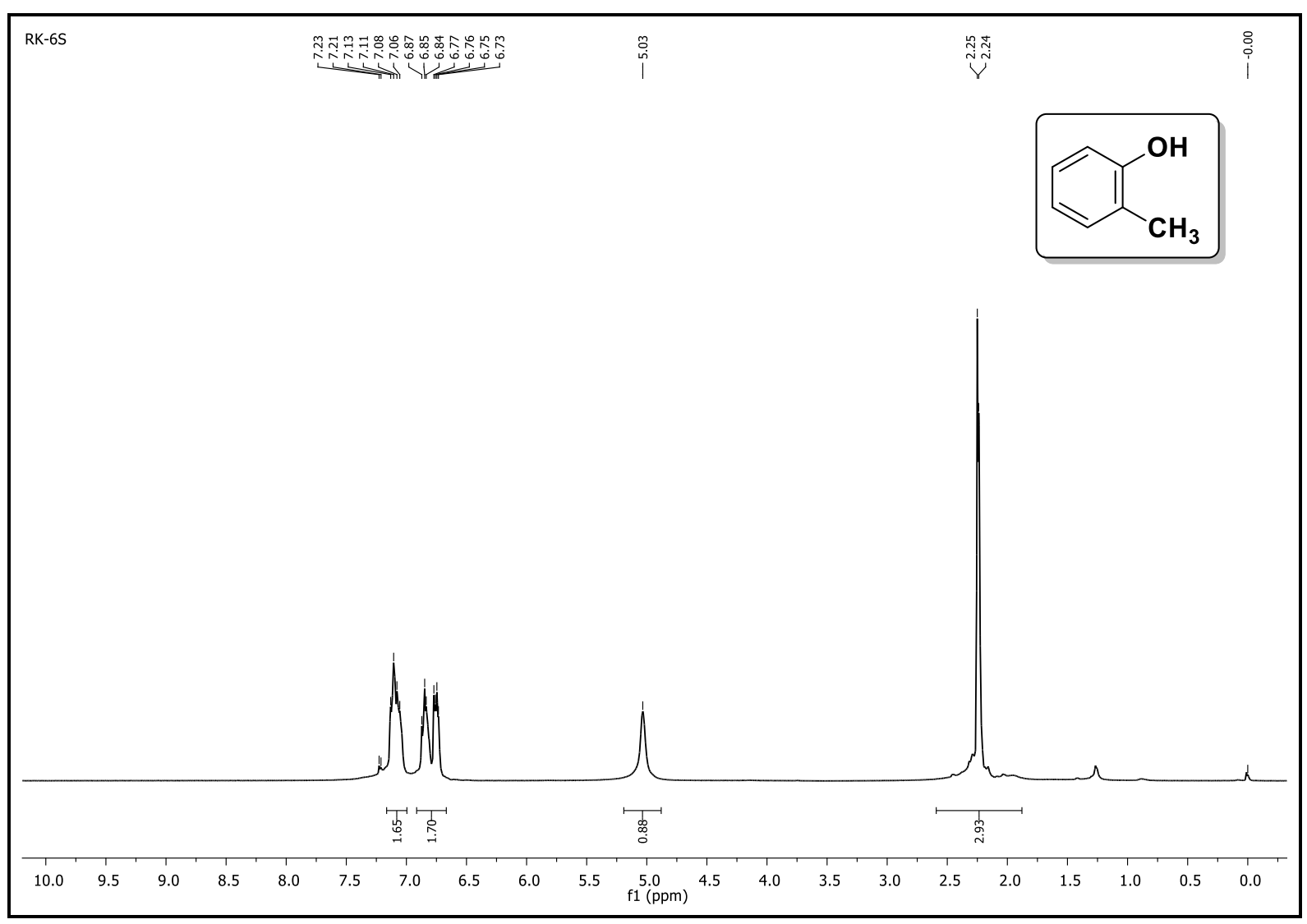

Fig. S24 ${ }^{1} \mathrm{H}$ NMR Spectrum of 2-methylphenol (2i) (300 MHz, $\mathrm{CDCl}_{3}$ )

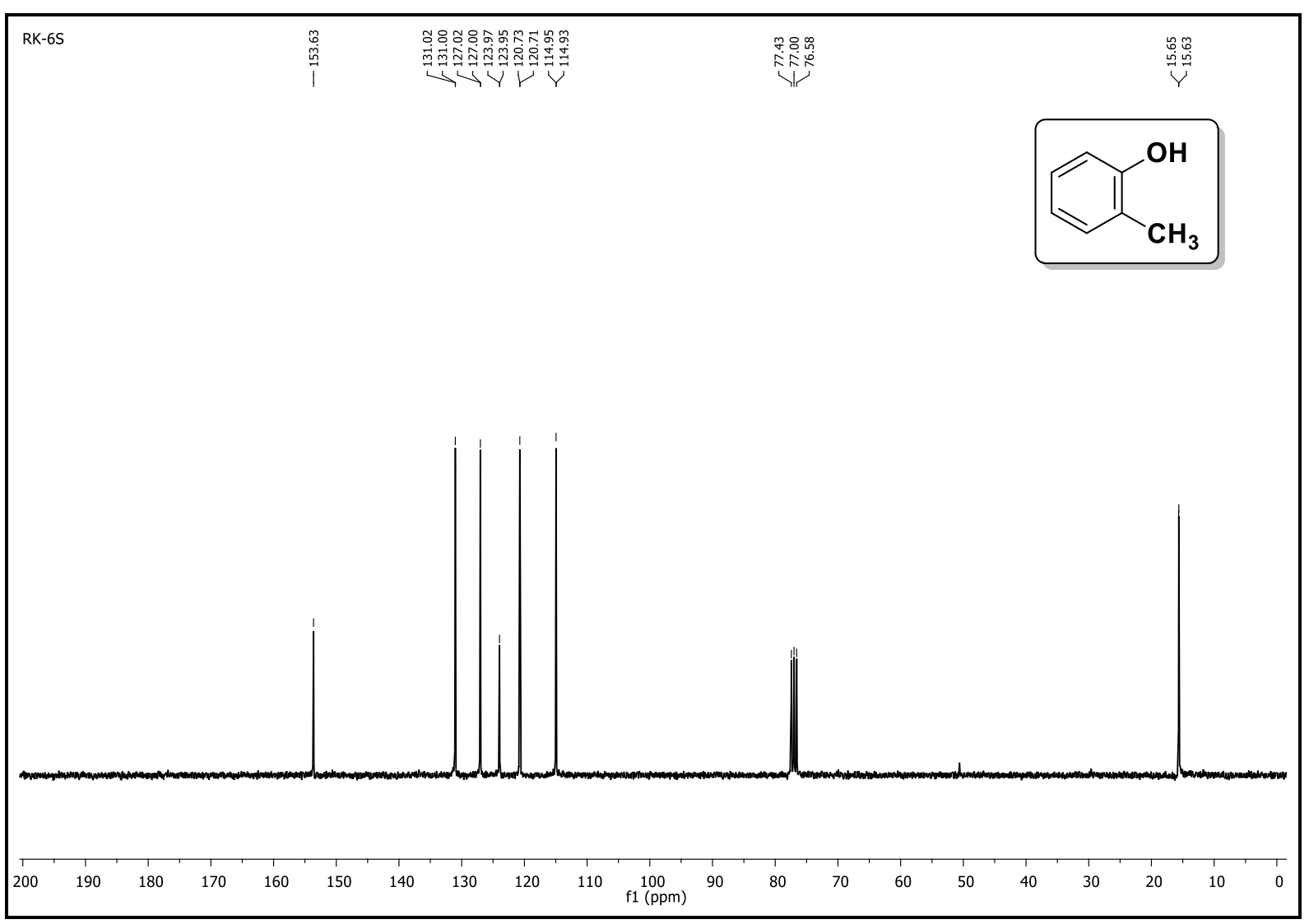

Fig. S25 ${ }^{13} \mathrm{C}$ NMR Spectrum of 2-methylphenol ((2i) $\left.75 \mathrm{MHz}, \mathrm{CDCl}_{3}\right)$ 


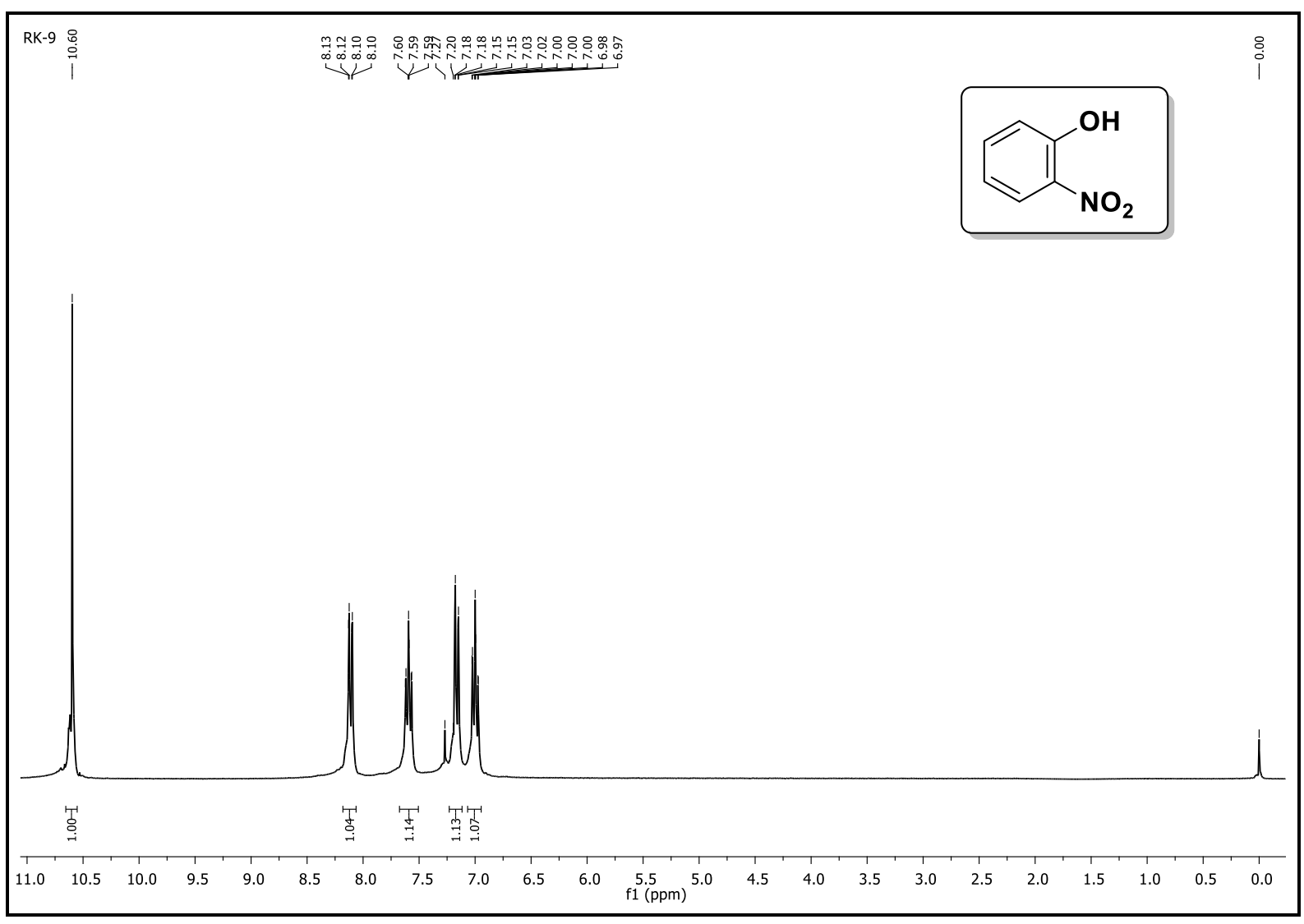

Fig. S26 ${ }^{1} \mathrm{H}$ NMR Spectrum of 2-Nitrophenol (2j) $\left(300 \mathrm{MHz}, \mathrm{CDCl}_{3}\right)$

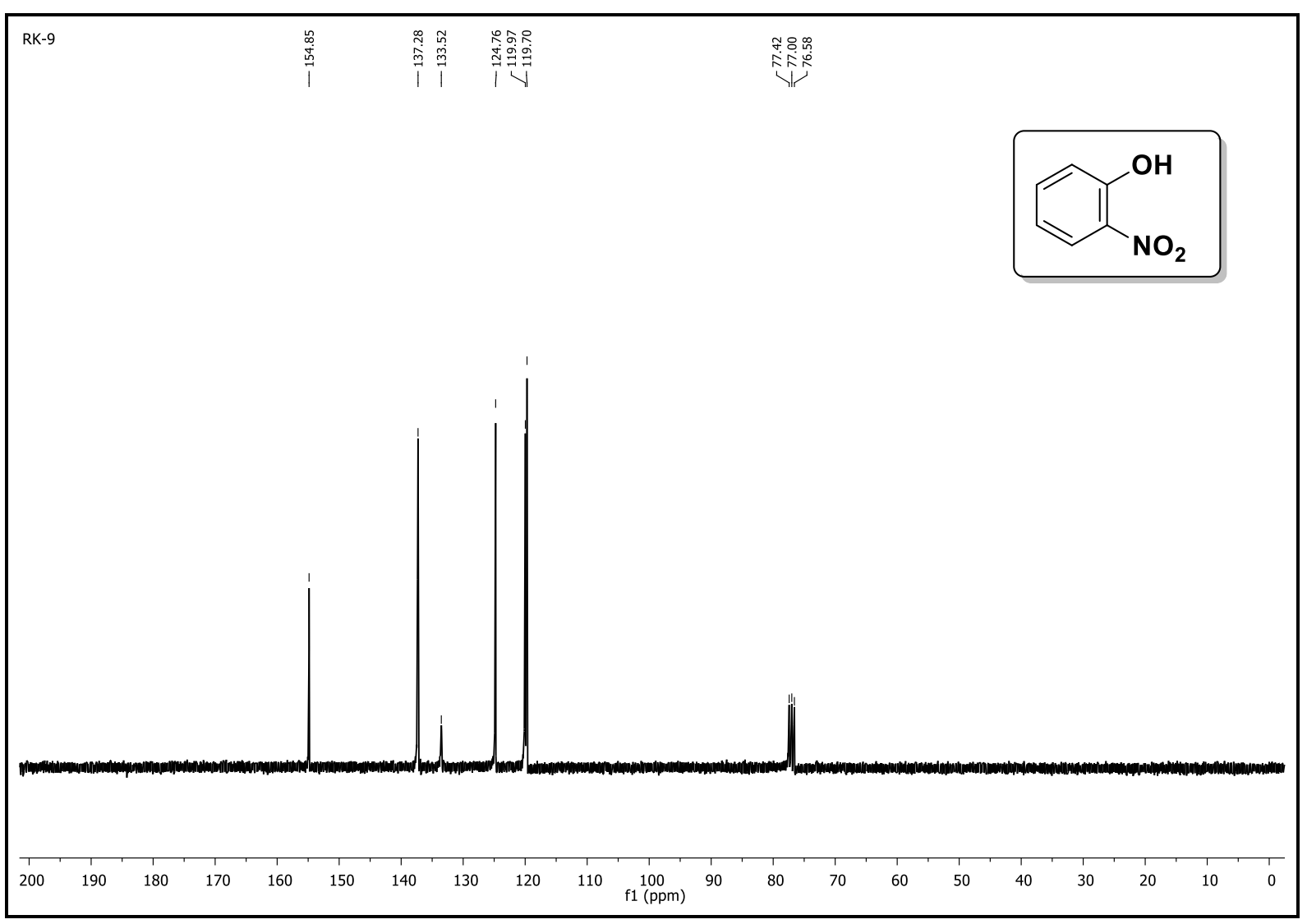

Fig. S27 ${ }^{13} \mathrm{C}$ NMR Spectrum of 2-Nitrophenol (2j) $\left(75 \mathrm{MHz}, \mathrm{CDCl}_{3}\right)$ 


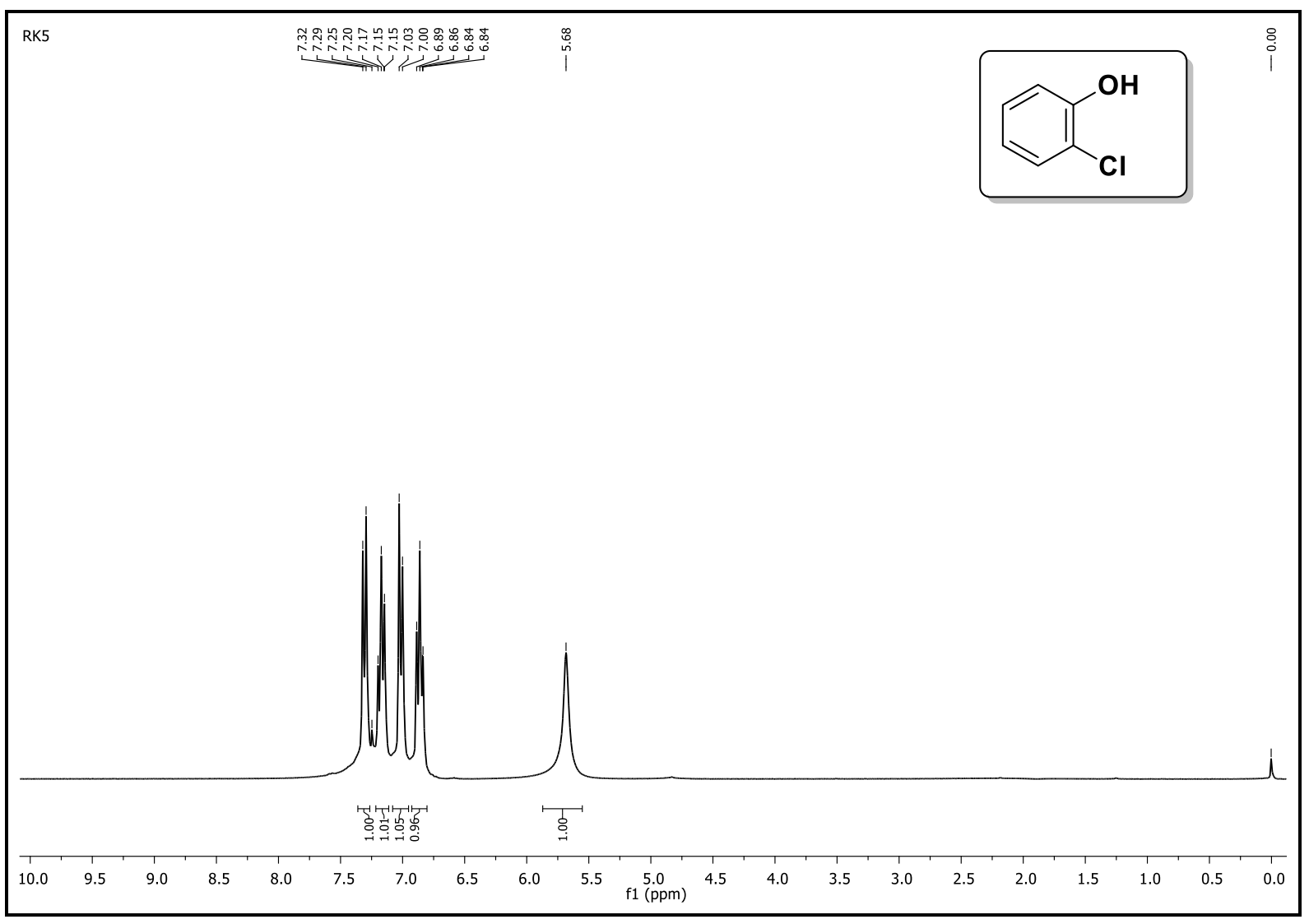

Fig. S28 ${ }^{1} \mathrm{H}$ NMR Spectrum of 2-chlorophenol (2k) (300 MHz, $\mathrm{CDCl}_{3}$ )

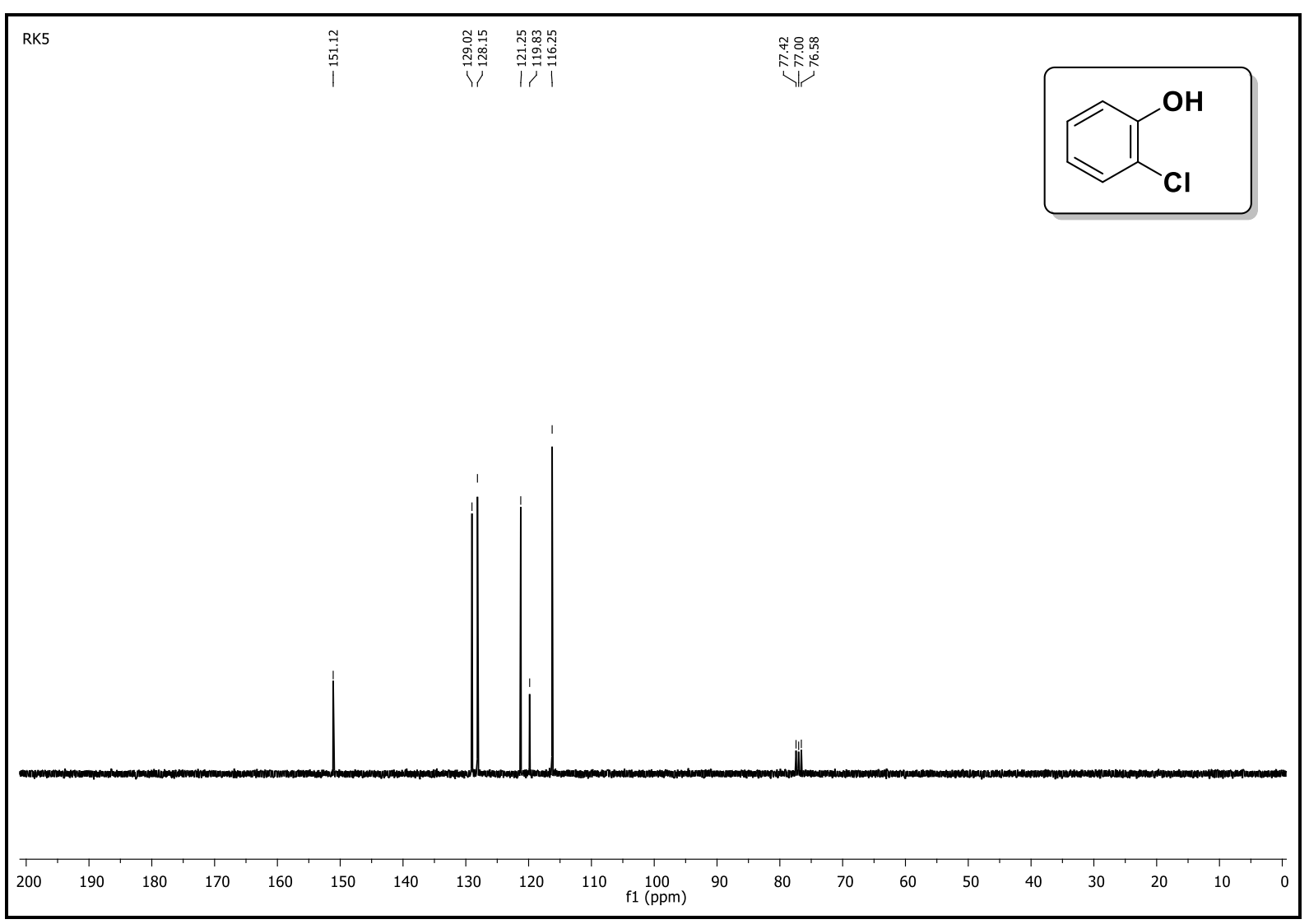

Fig. S29 ${ }^{13} \mathrm{C}$ NMR Spectrum of 2-chlorophenol (2k) (75 MHz, $\left.\mathrm{CDCl}_{3}\right)$ 


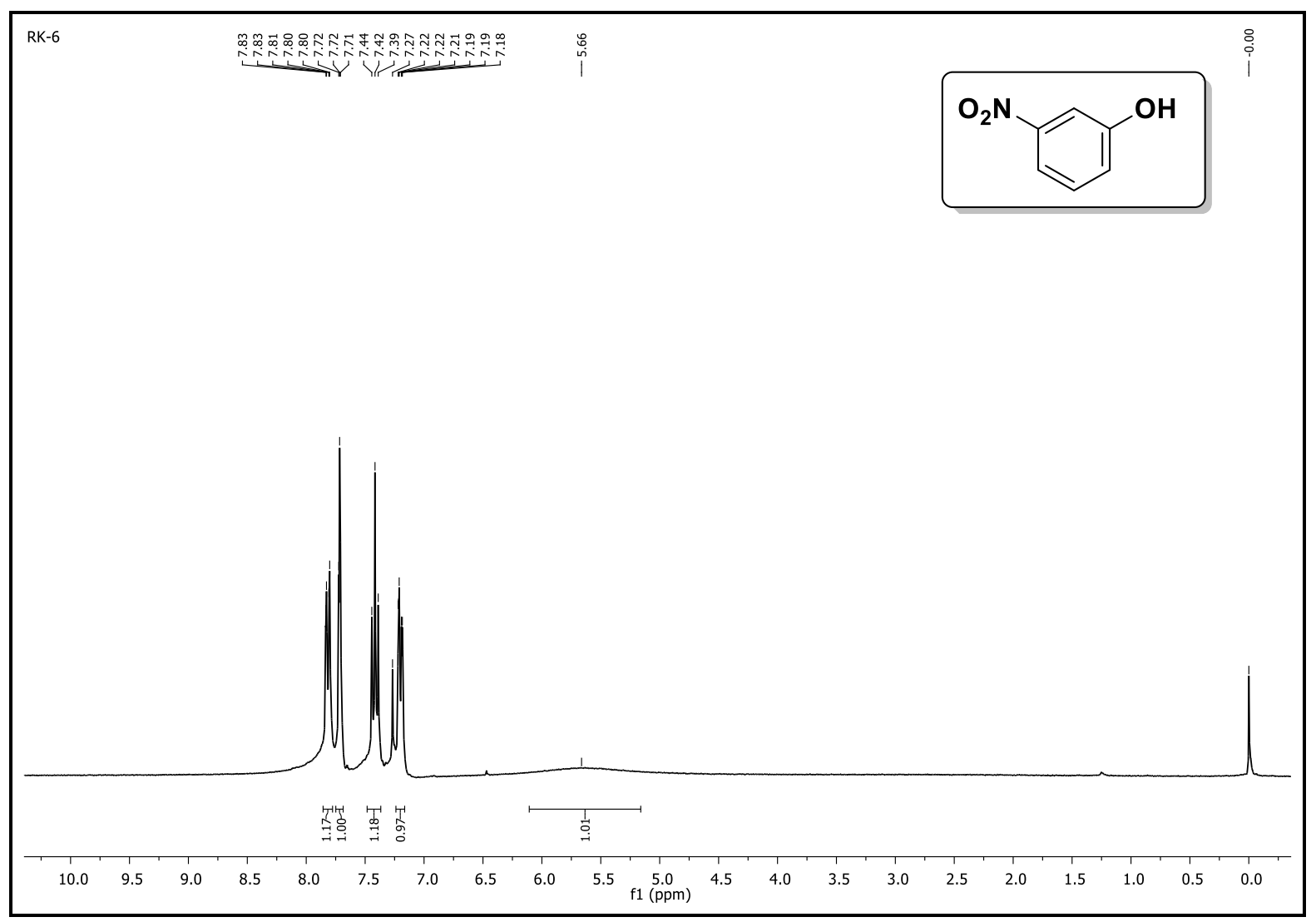

Fig. S30 ${ }^{1} \mathrm{H}$ NMR Spectrum of 3-Nitrophenol (2l) (300 MHz, $\left.\mathrm{CDCl}_{3}\right)$

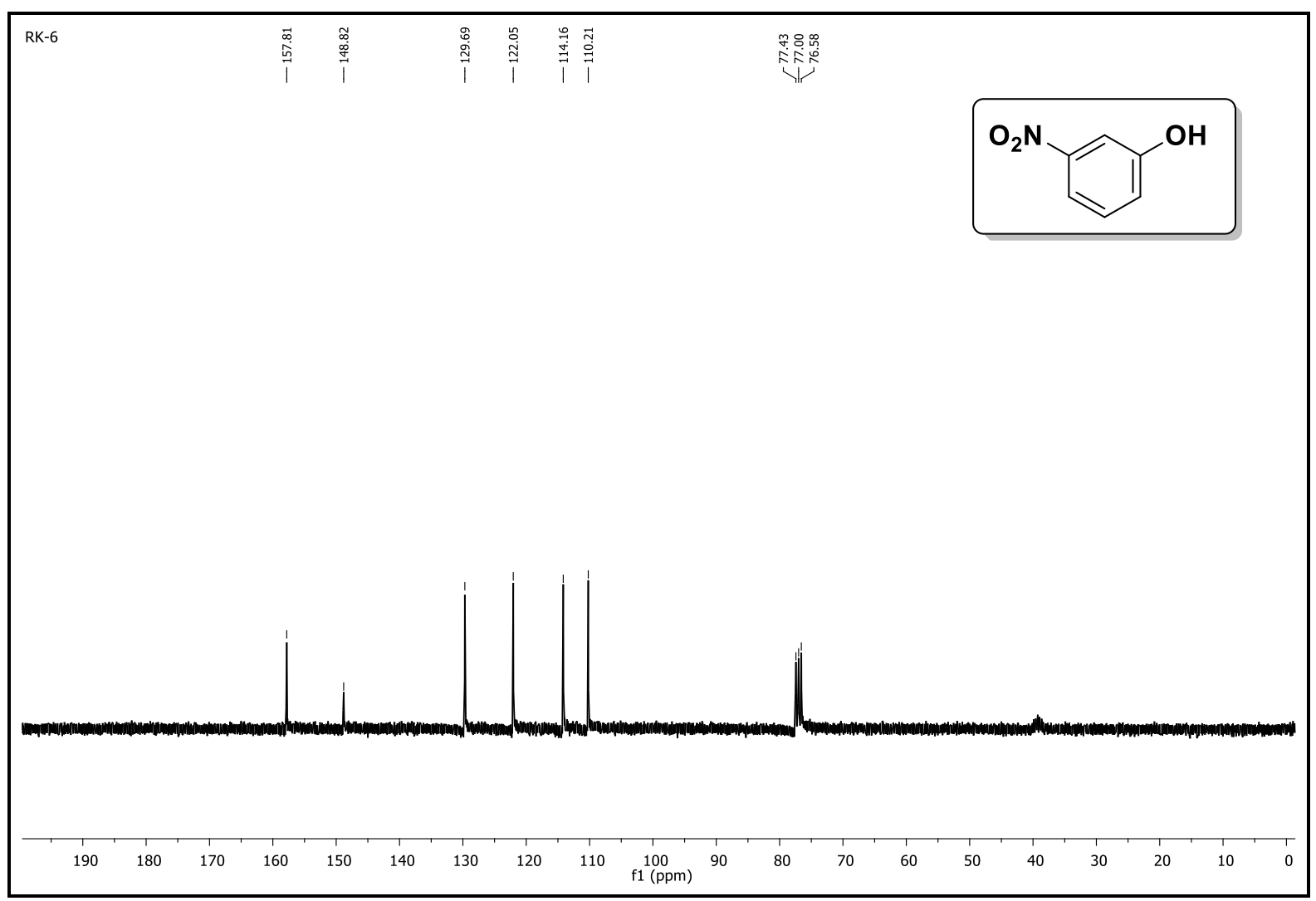

Fig. S31 ${ }^{13} \mathrm{C}$ NMR Spectrum of 3-Nitrophenol (2I) (75 MHz, $\left.\mathrm{CDCl}_{3}\right)$ 


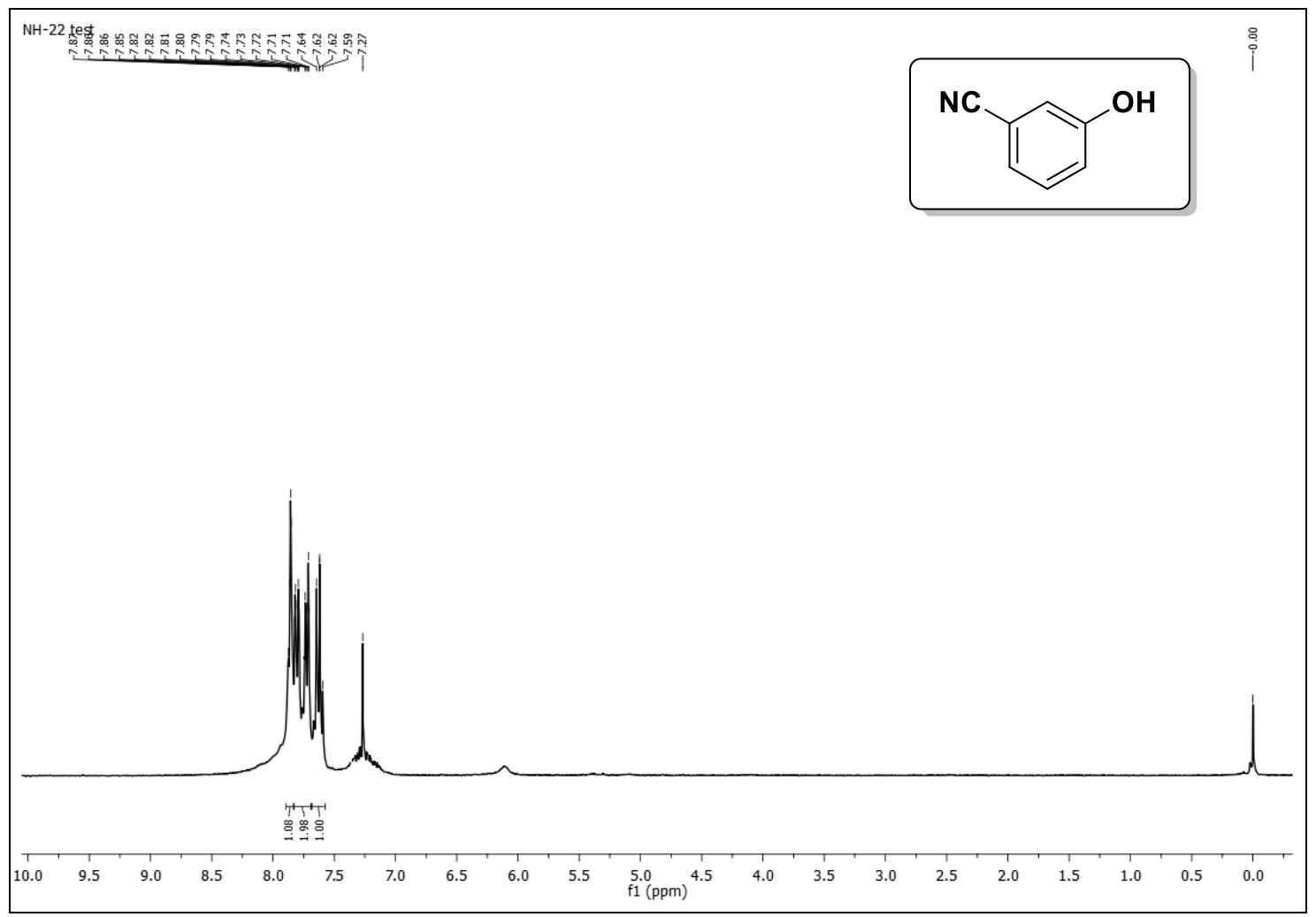

Fig. S32 ${ }^{1} \mathrm{H}$ NMR Spectrum of 3-hydroxybaenzonaaitrile (2m) (300 MHz, $\left.\mathrm{CDCl}_{3}\right)$

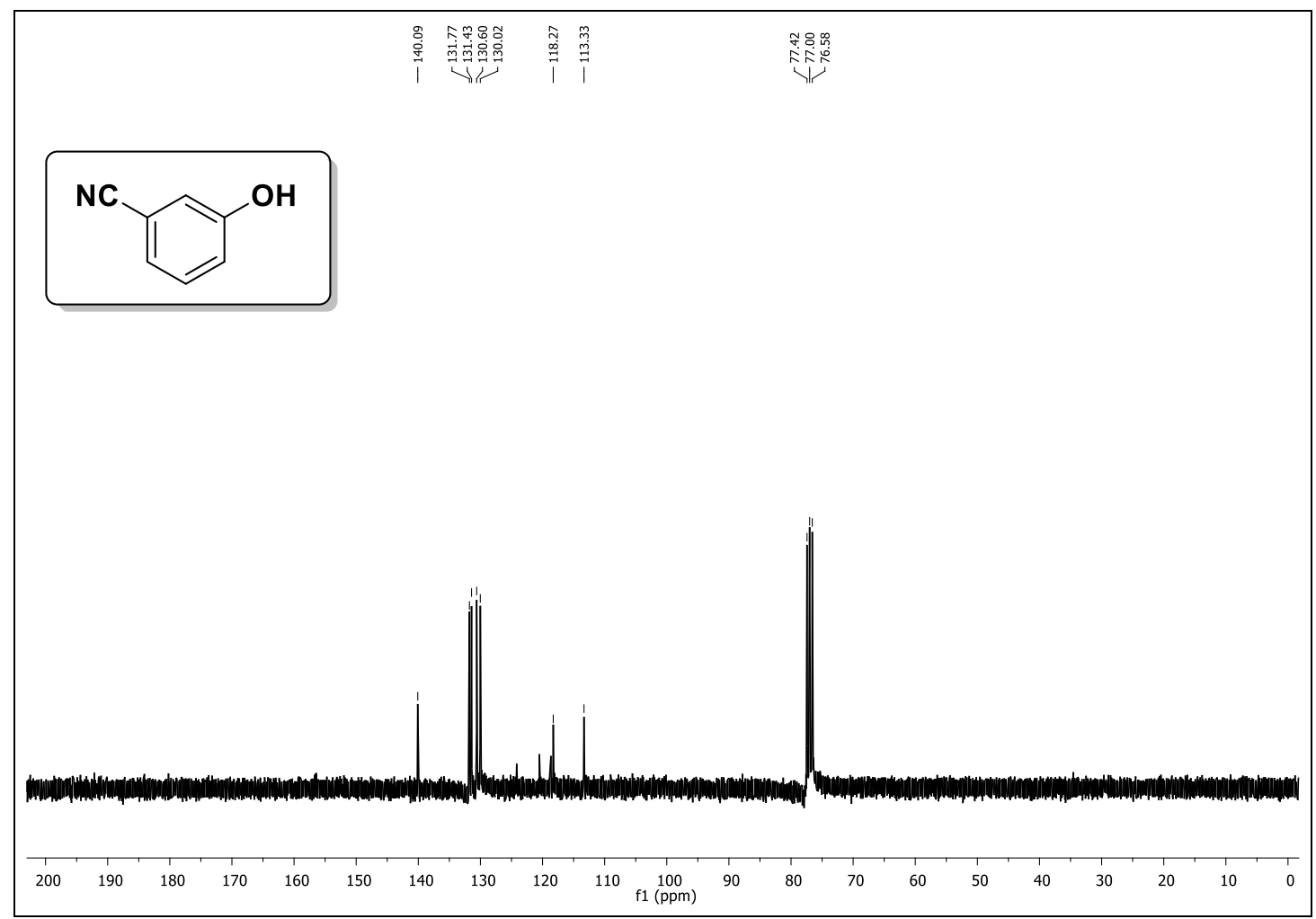

Fig. S33 ${ }^{13} \mathrm{C}$ NMR Spectrum of 3-hydroxybaenzonaaitrile (2m) (75 MHz, $\mathrm{CDCl}_{3}$ ) 


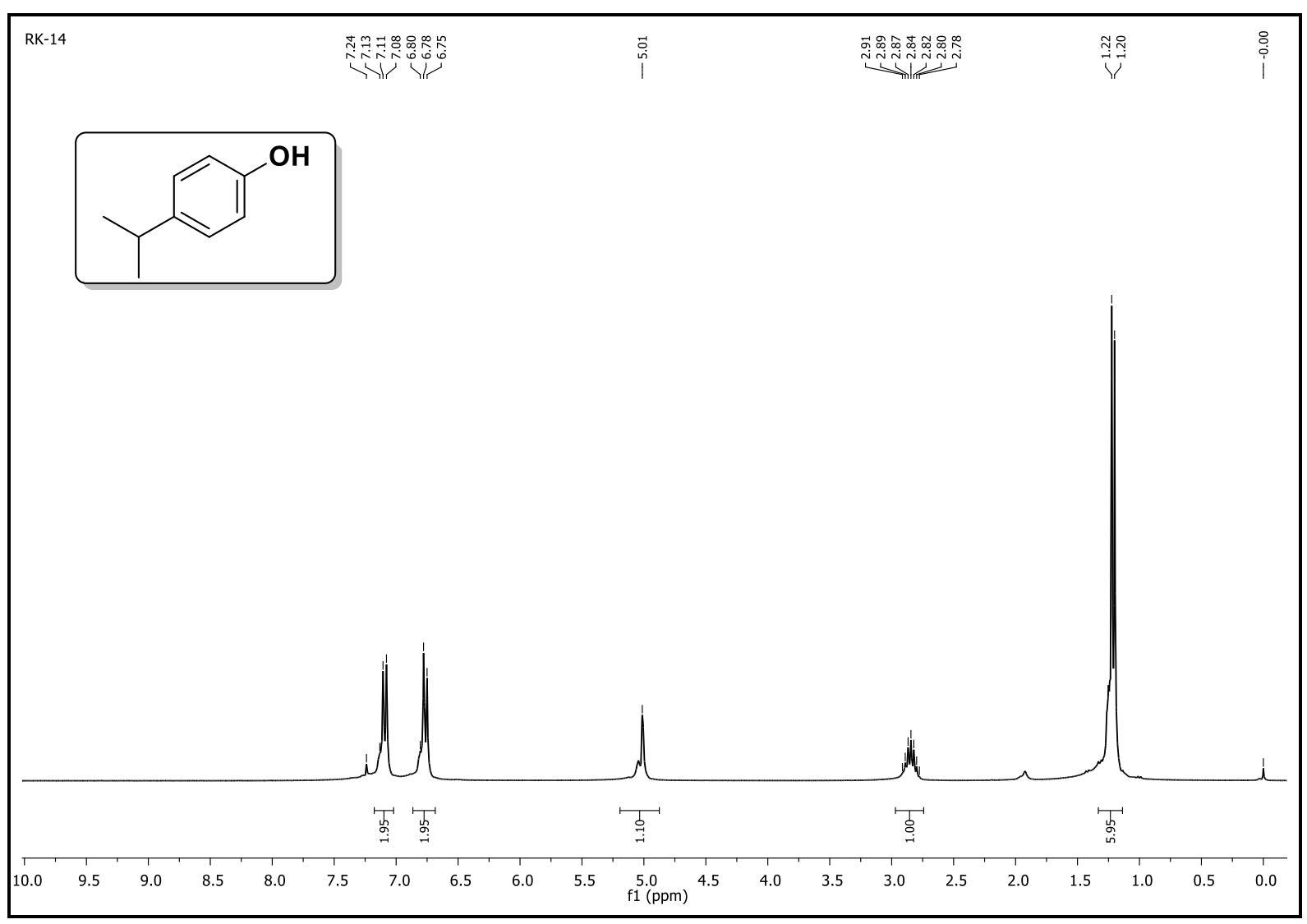

Fig. S34 ${ }^{1} \mathrm{H}$ NMR Spectrum of 4-isopropylphenol (2n) (300 MHz, $\left.\mathrm{CDCl}_{3}\right)$

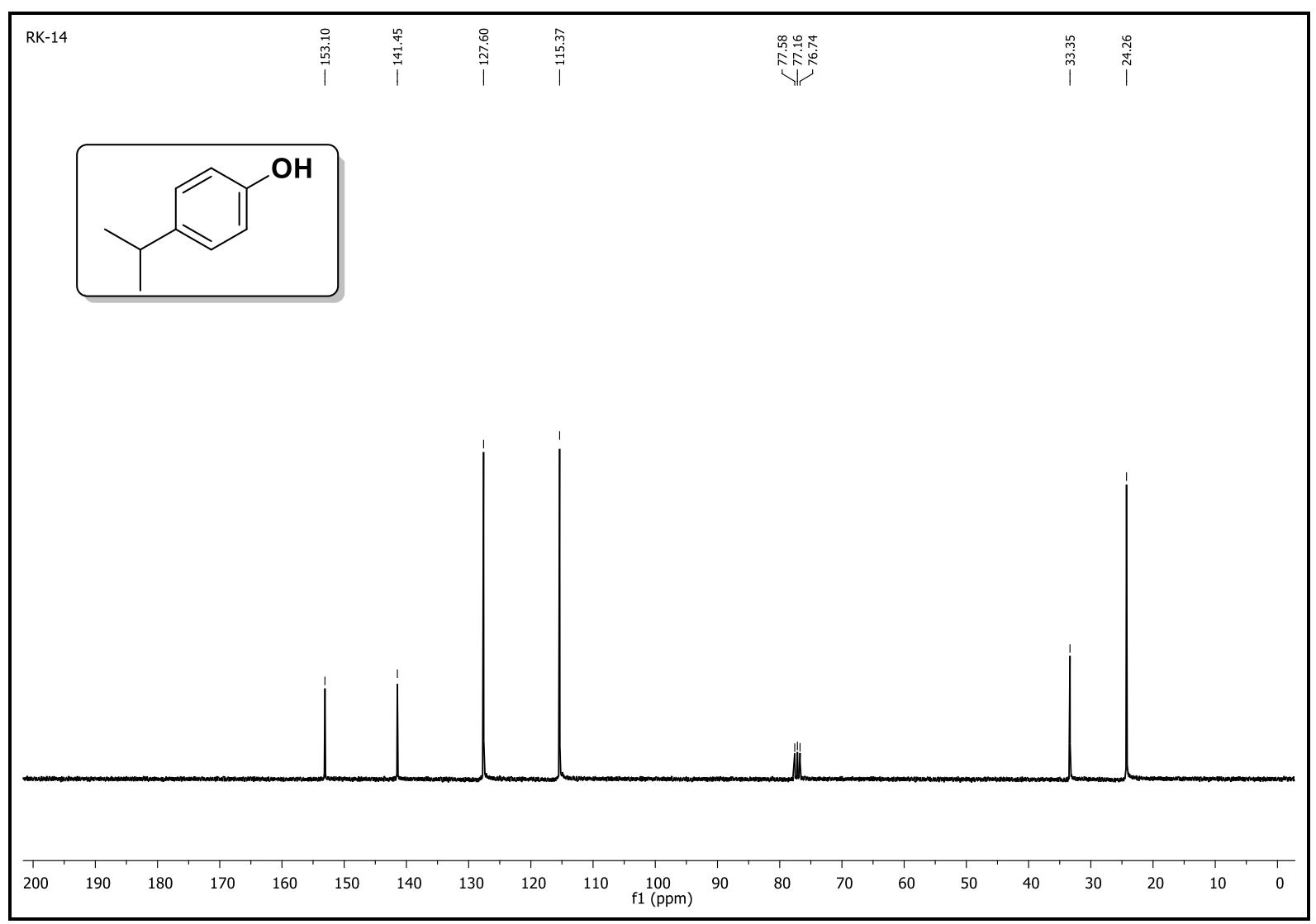

Fig. S35 ${ }^{13} \mathrm{C}$ NMR Spectrum of 4-isopropylphenol (2n) $\left(75 \mathrm{MHz}, \mathrm{CDCl}_{3}\right)$ 


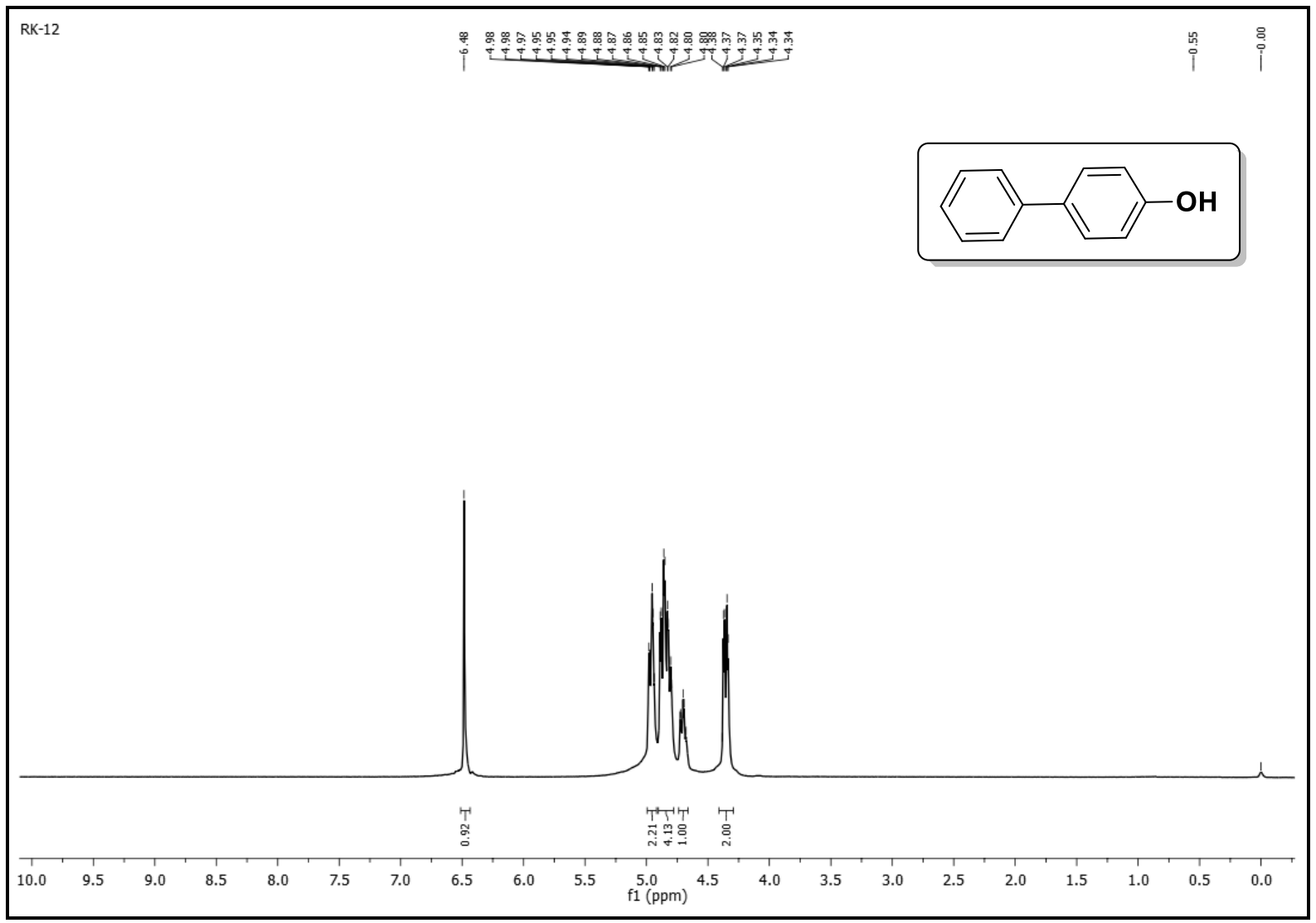

Fig. S36 ${ }^{1} \mathrm{H}$ NMR Spectrum of [1,1'-biphenyl]-4-ol (2o) (300 MHz, $\left.\mathrm{CDCl}_{3}\right)$

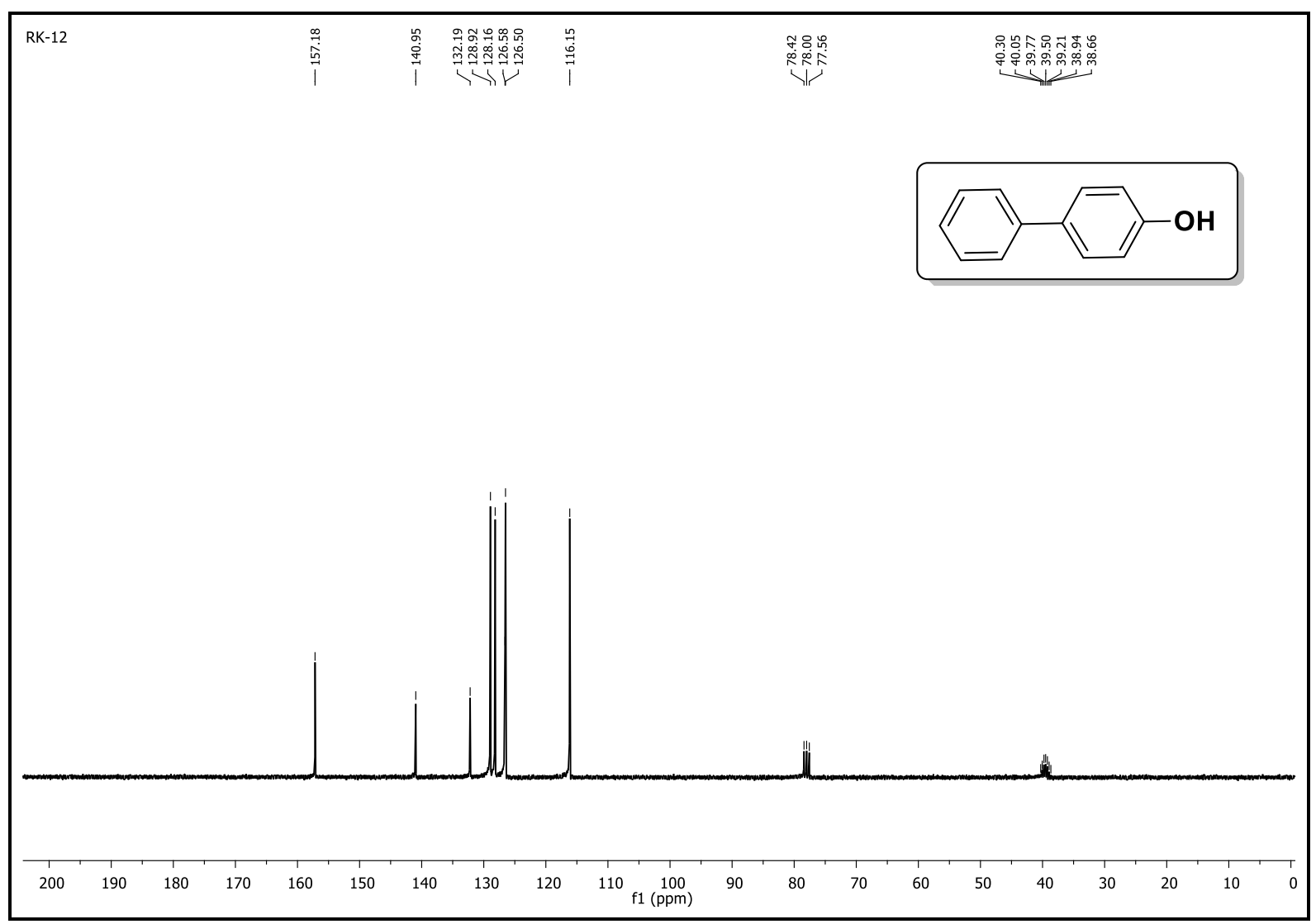

Fig. $\mathbf{S 3 7}{ }^{13} \mathrm{C}$ NMR Spectrum of $\left[1,1^{\prime}\right.$-biphenyl]-4-ol (2o) $\left(75 \mathrm{MHz}, \mathrm{CDCl}_{3}\right)$ 


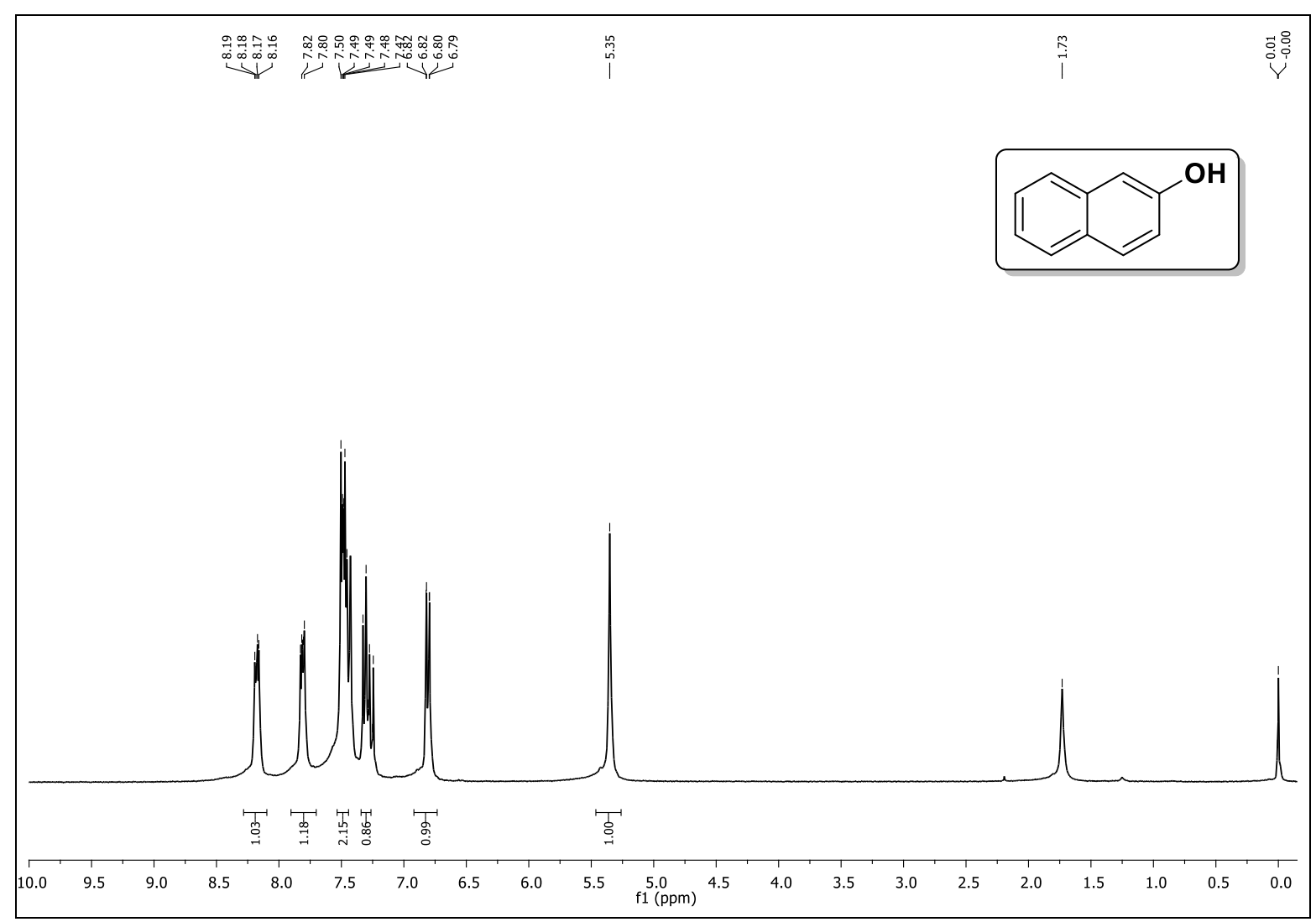

Fig. S38 ${ }^{13} \mathrm{C}$ NMR Spectrum of naphthalen-2-ol (2p) (300 MHz, $\left.\mathrm{CDCl}_{3}\right)$

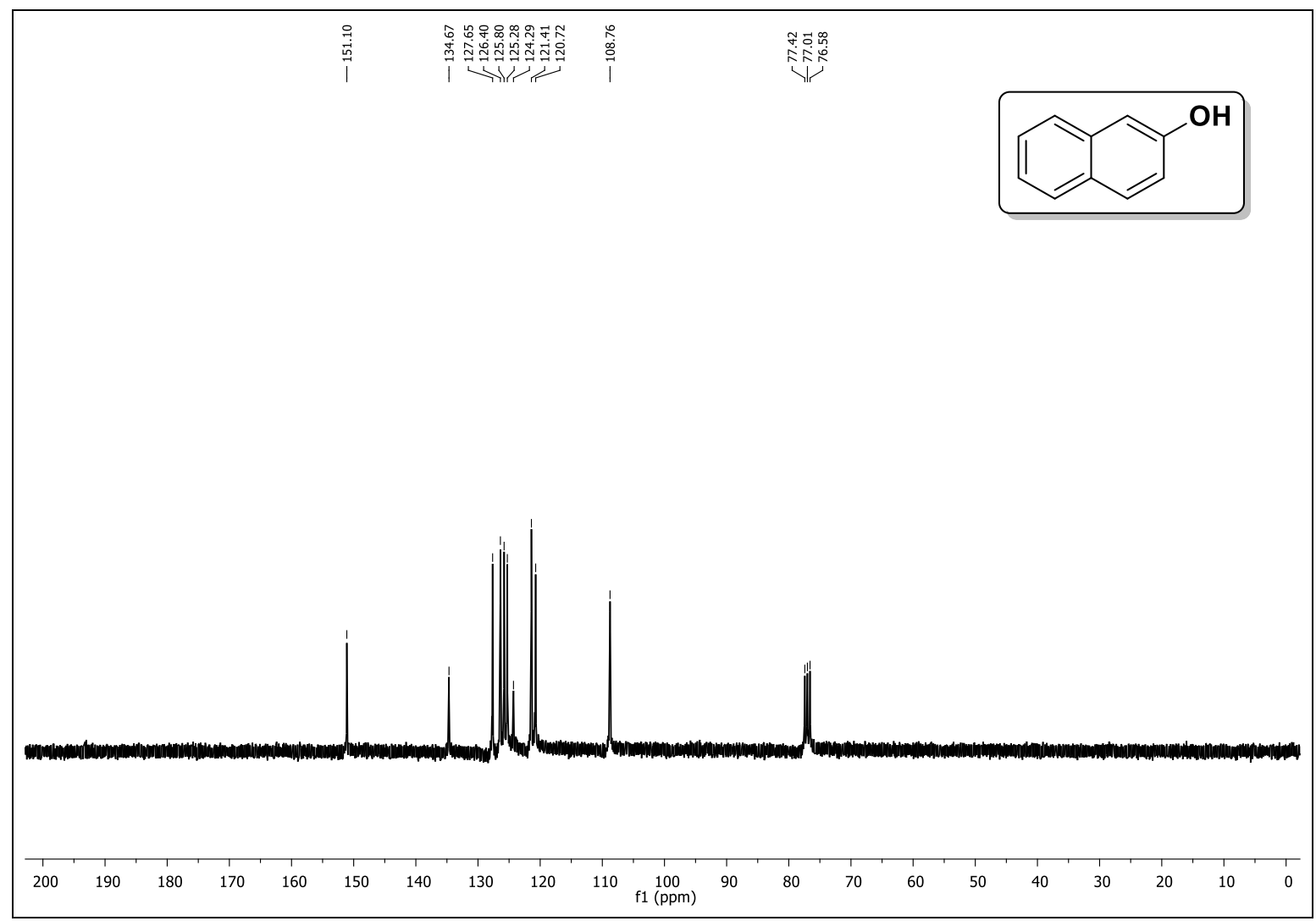

Fig. S39 ${ }^{13} \mathrm{C}$ NMR Spectrum of naphthalen-2-ol (2p) $\left(75 \mathrm{MHz}, \mathrm{CDCl}_{3}\right)$ 


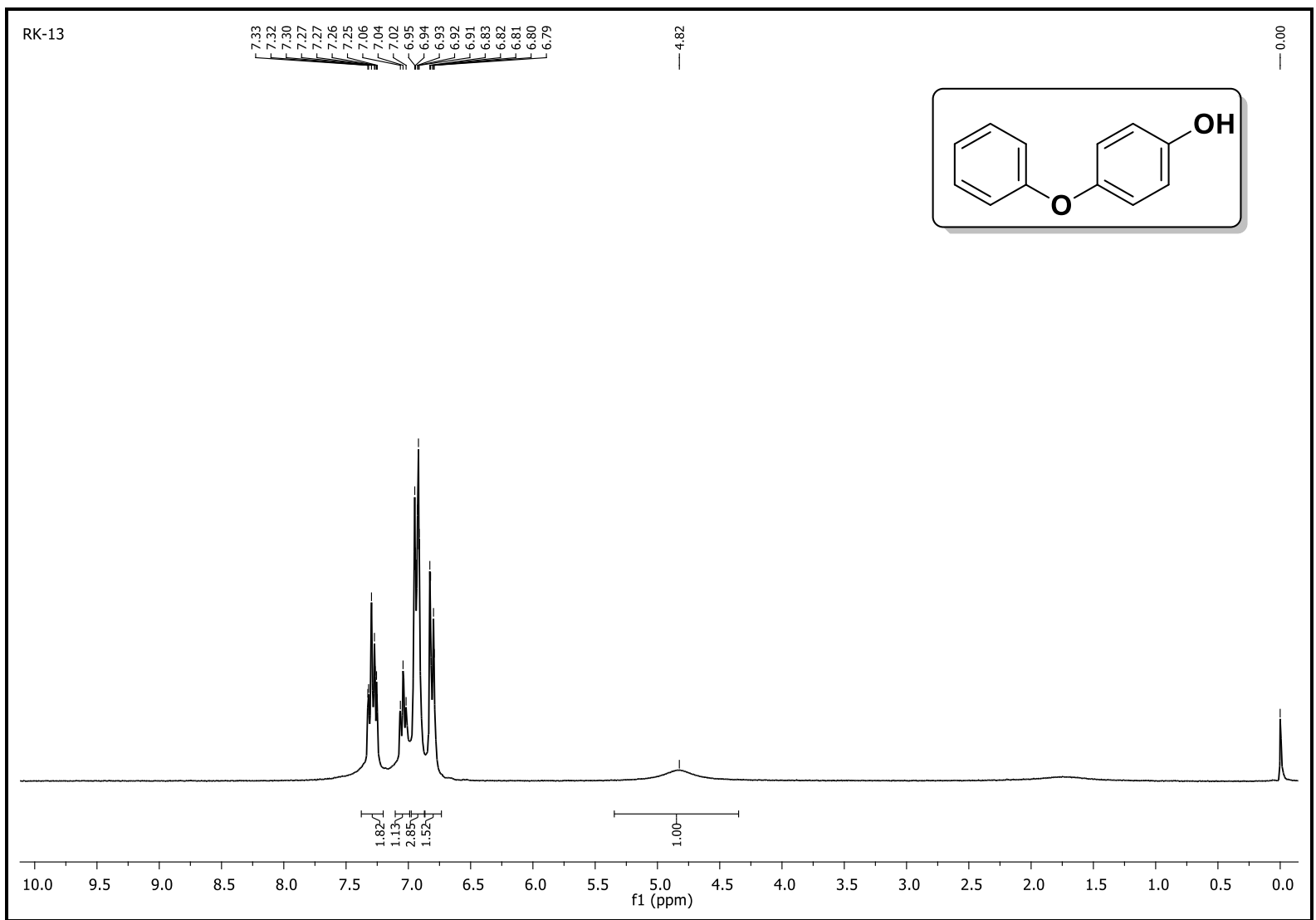

Fig. S40 ${ }^{1} \mathrm{H}$ NMR Spectrum of 4-phenoxyphenol (2q) (300 MHz, $\left.\mathrm{CDCl}_{3}\right)$

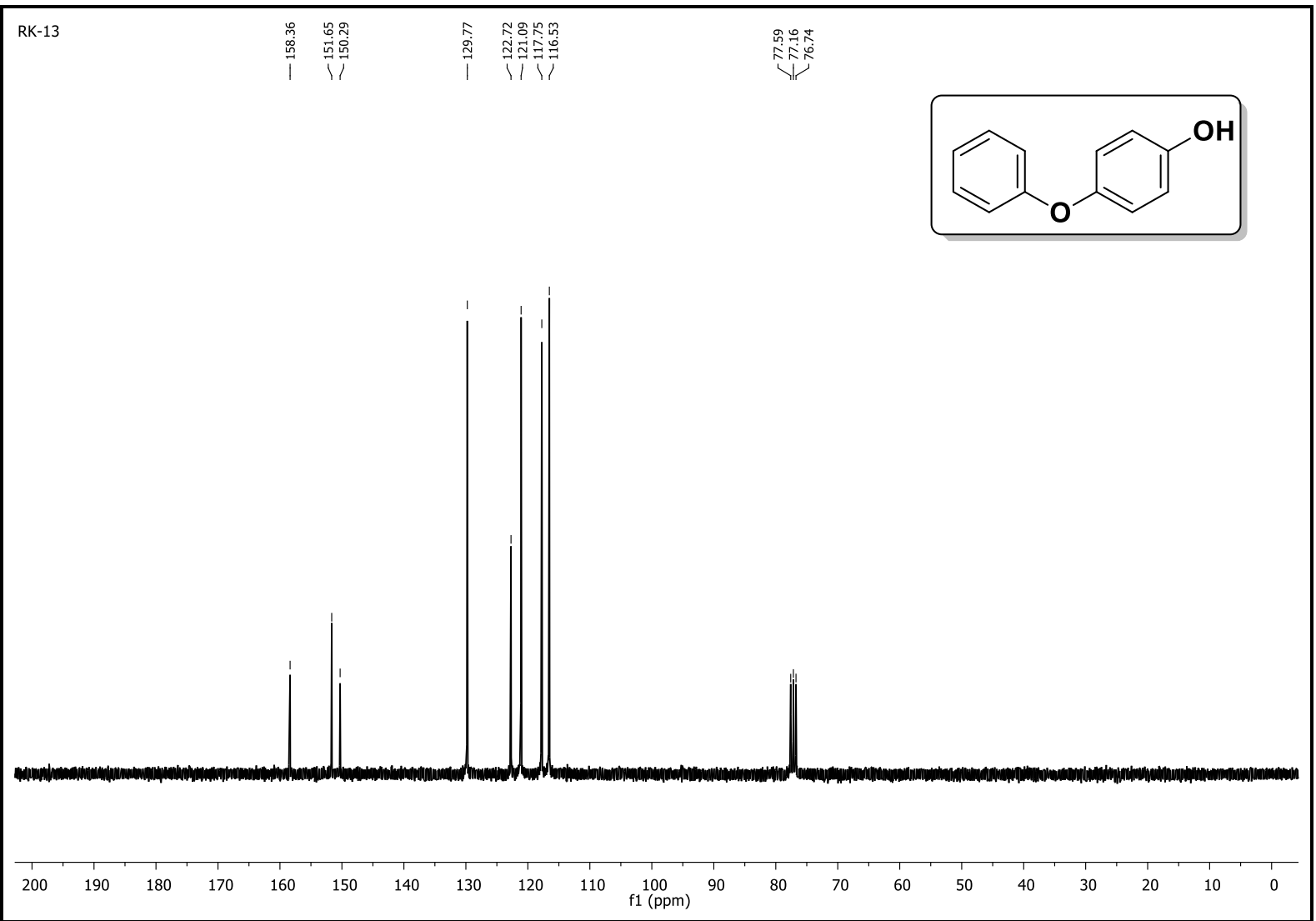

Fig. S41 ${ }^{13} \mathrm{C}$ NMR Spectrum of 4-phenoxyphenol (2q) $\left(75 \mathrm{MHz}, \mathrm{CDCl}_{3}\right)$ 


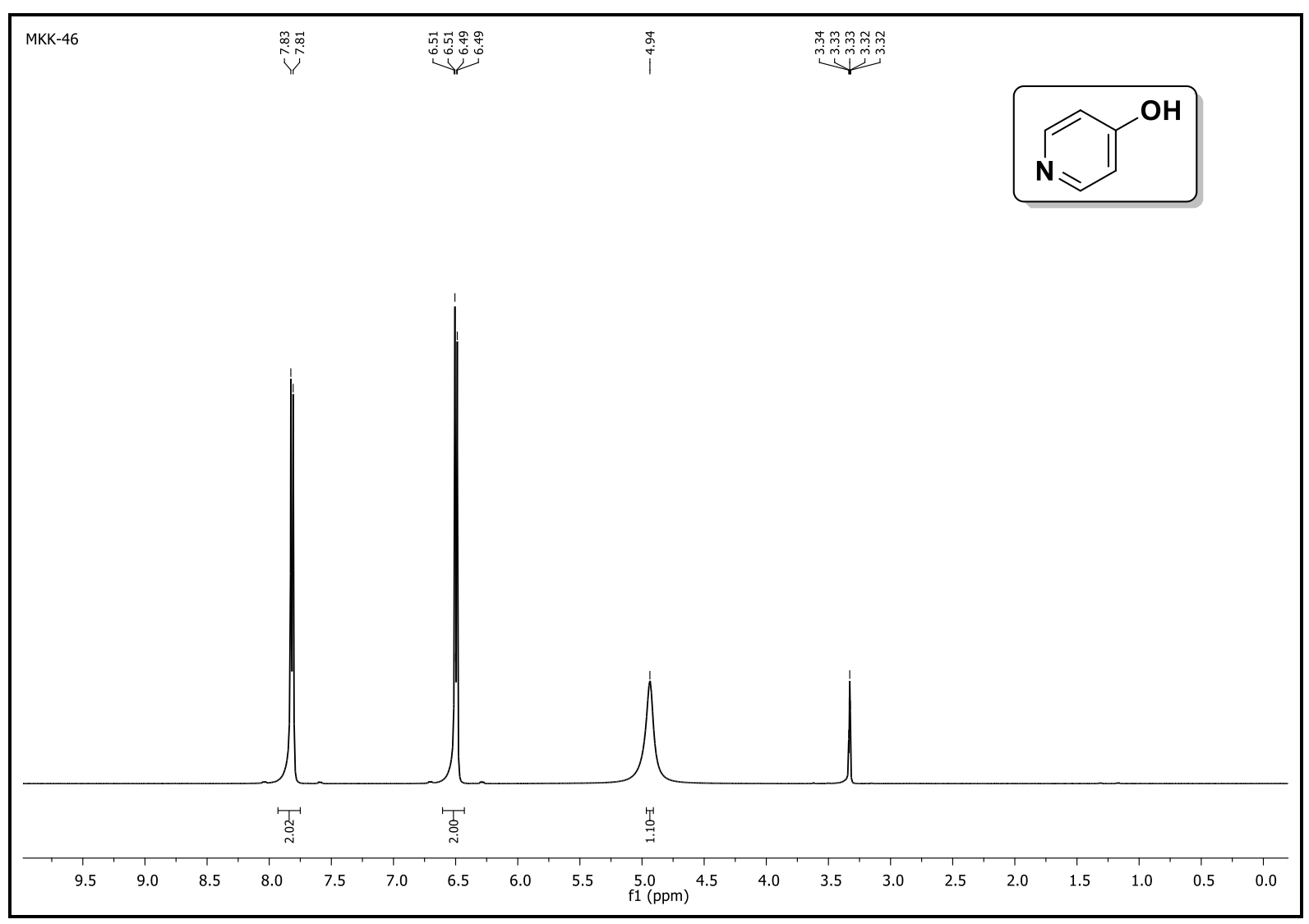

Fig. S42 ${ }^{1}$ H NMR Spectrum of pyridin-4-ol (2r) (400 MHz, MeOD)

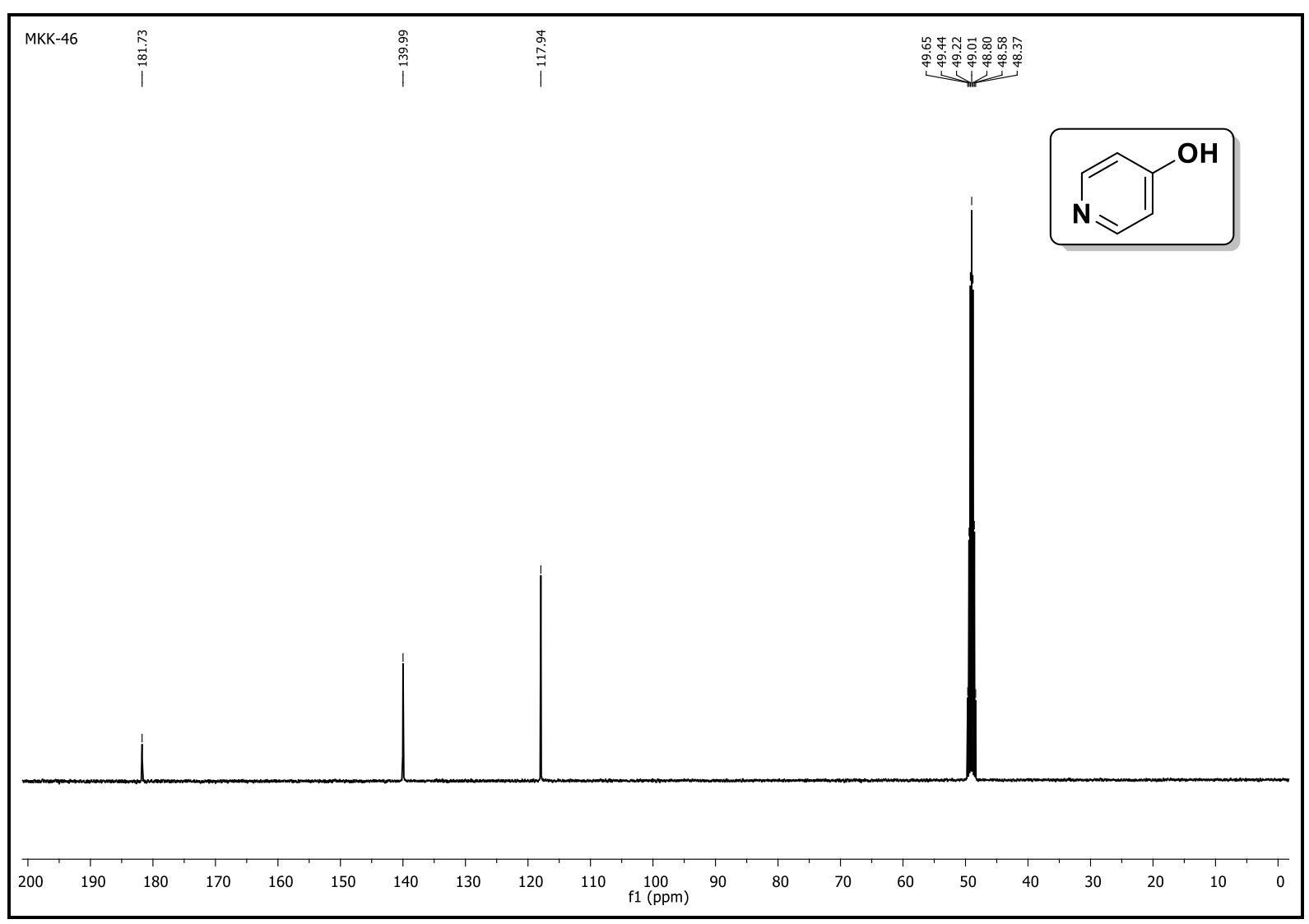

Fig. $\mathbf{S 4 3}{ }^{13} \mathrm{C}$ NMR Spectrum of pyridin-4-ol (2r) (101 MHz, MeOD) 


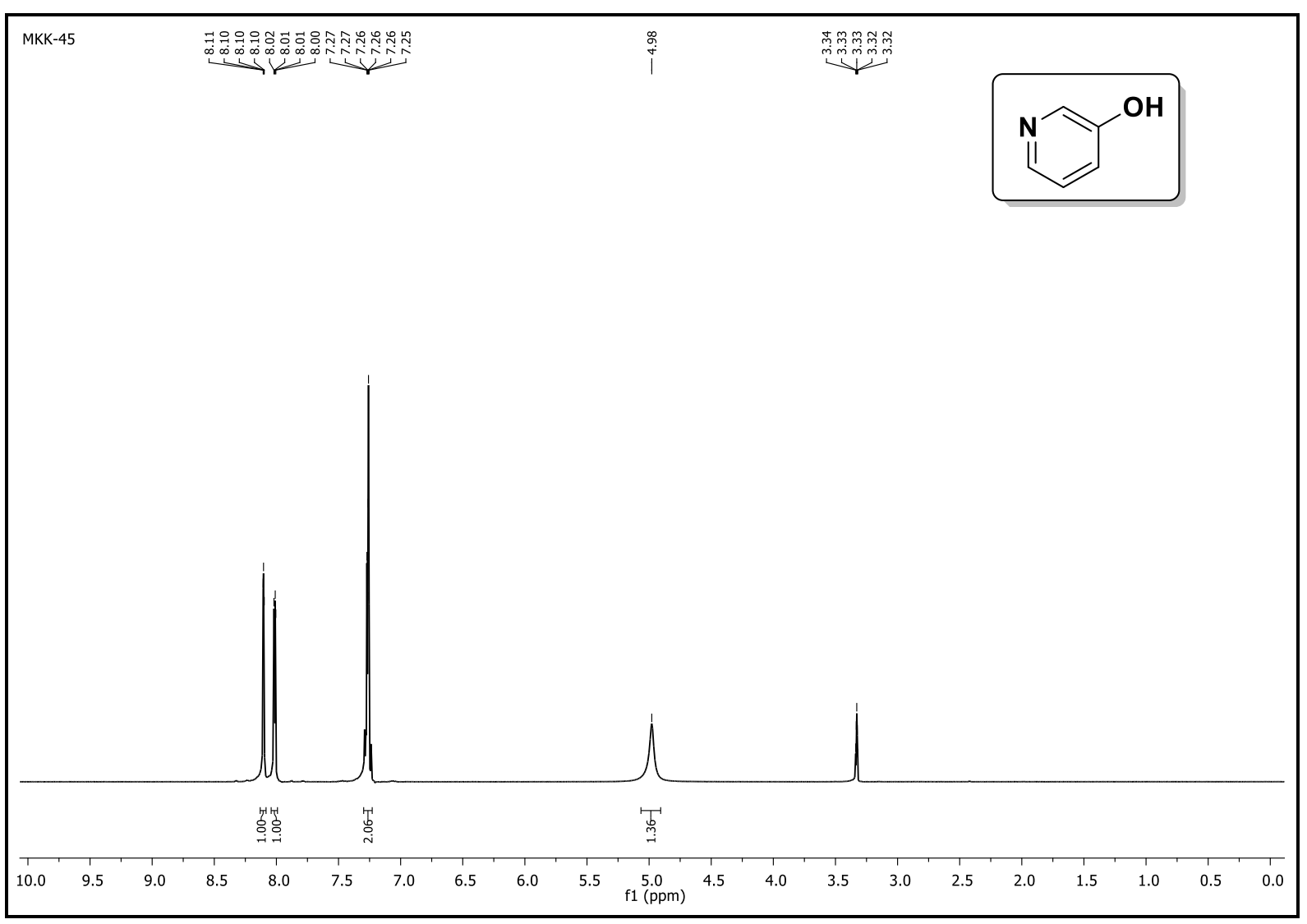

Fig. $\mathbf{S 4 4}{ }^{1}$ H NMR Spectrum of pyridin-3-ol (2s) (400 MHz, MeOD)

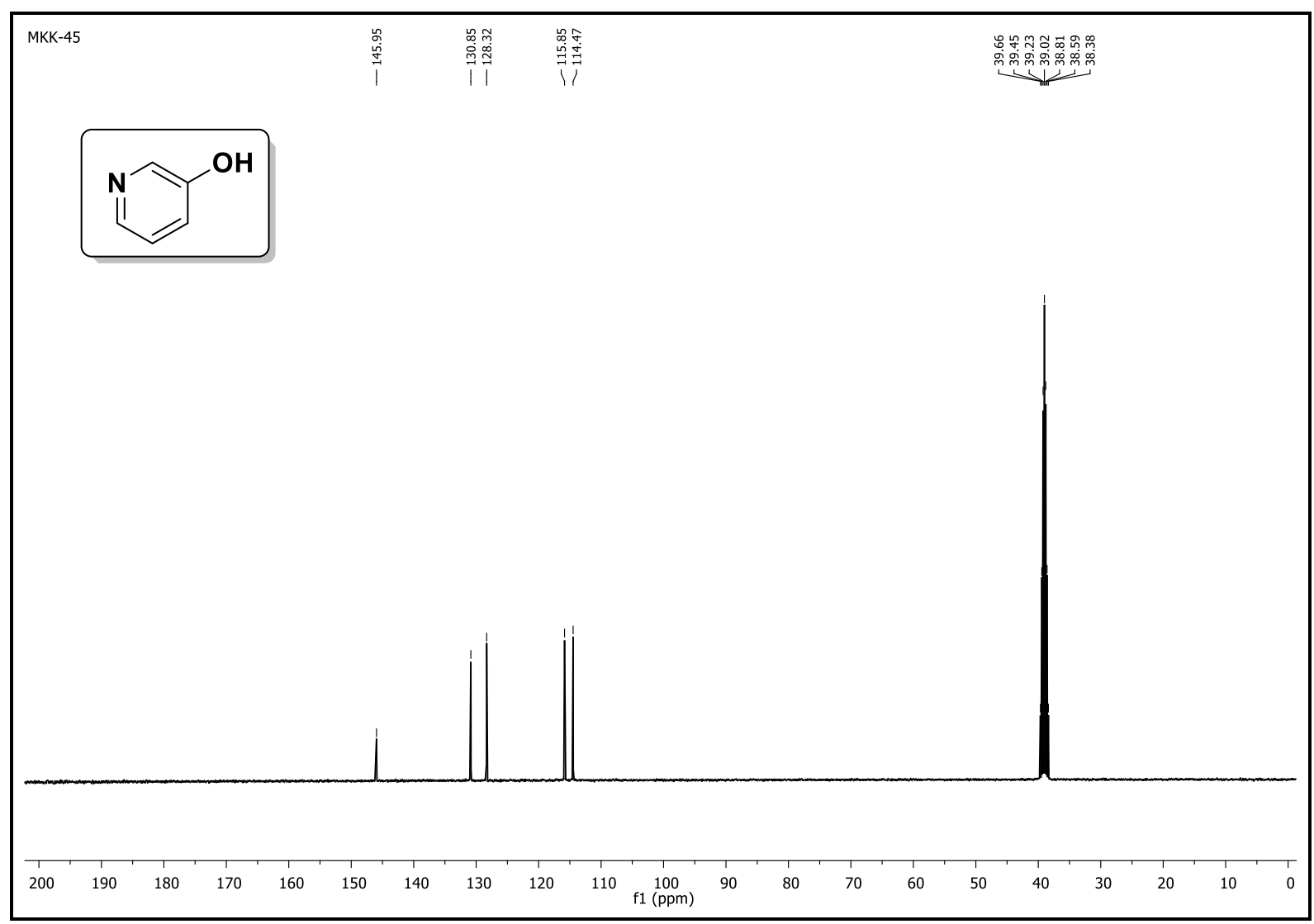

Fig. S45 ${ }^{13} \mathrm{C}$ NMR Spectrum of pyridin-3-ol (2s) (101 MHz, MeOD) 


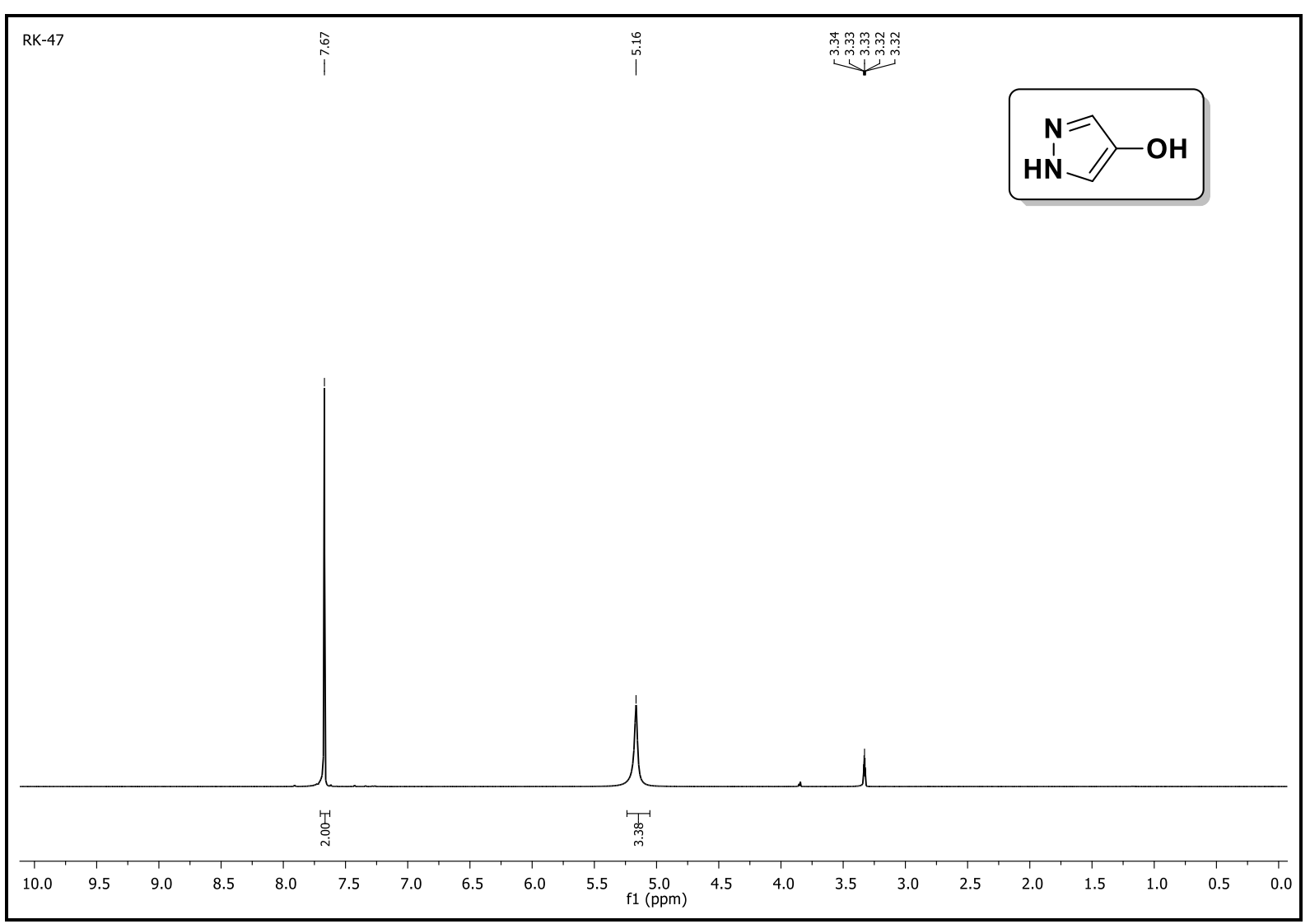

Fig. S46 ${ }^{1}$ H NMR Spectrum of 4-Hydroxypyrazole (2t) (400 MHz, MeOD)

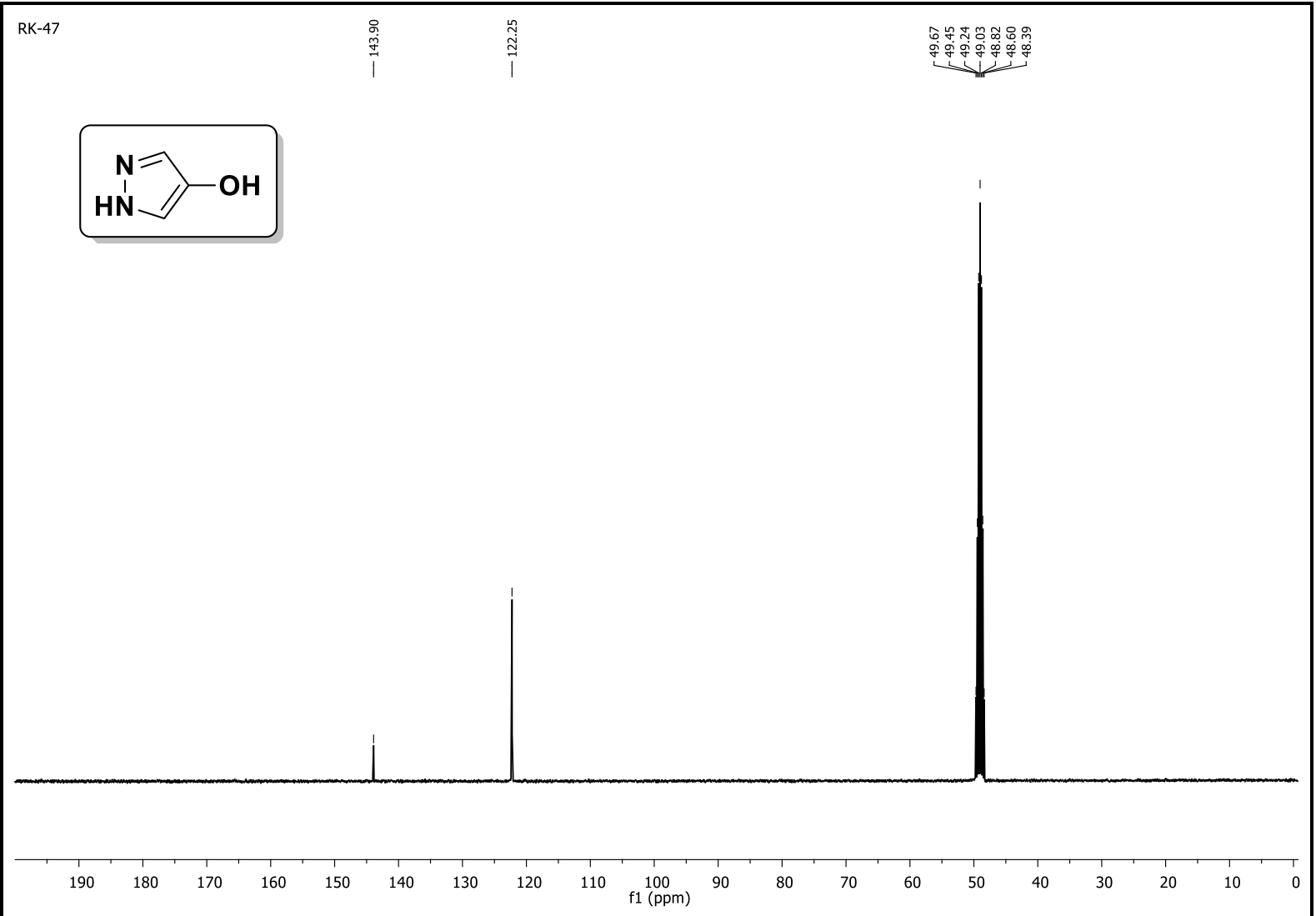

Fig. S47 ${ }^{13} \mathrm{C}$ NMR Spectrum of 4-Hydroxypyrazole (2t) (101 MHz, MeOD) 


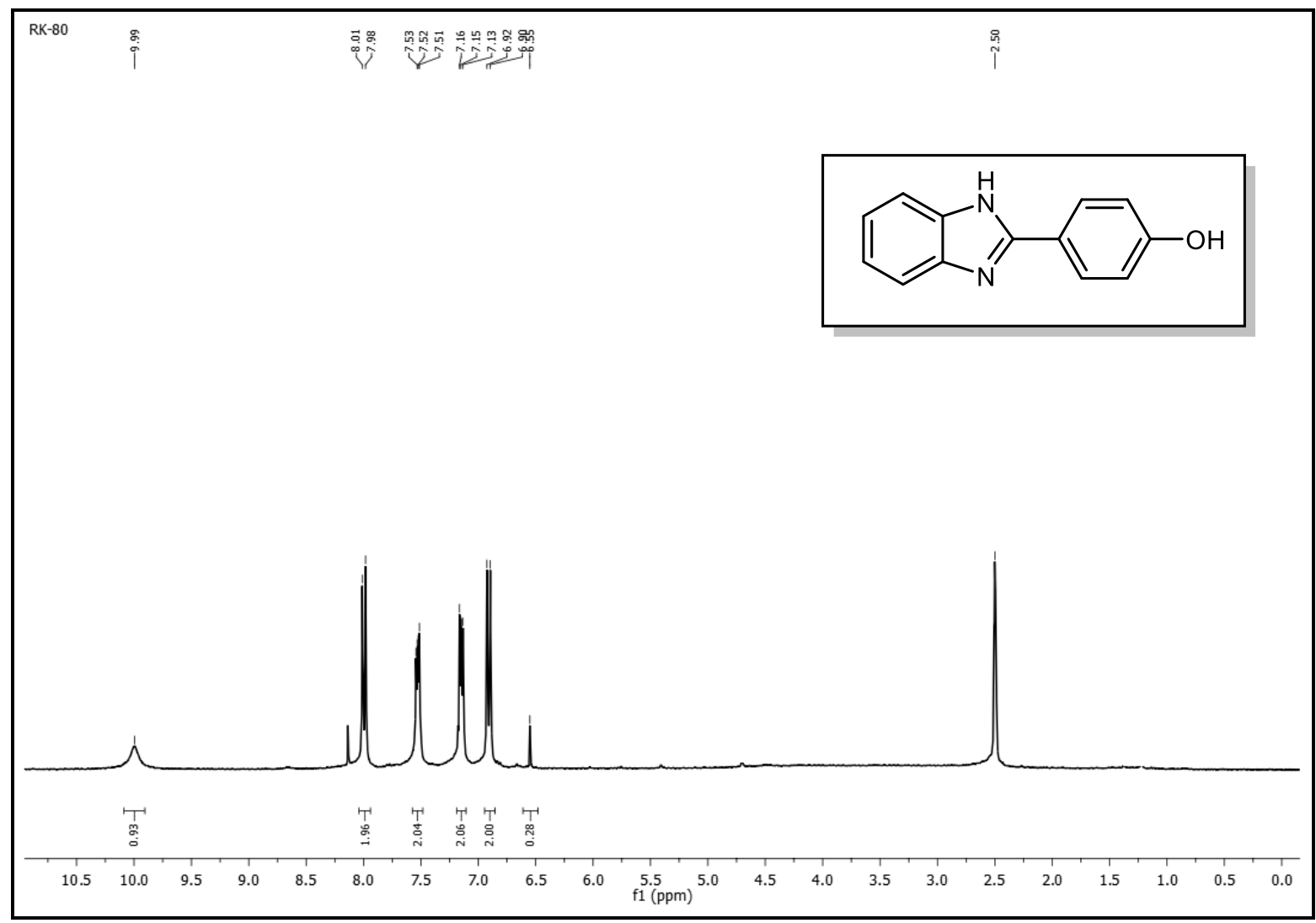

Fig. S48 ${ }^{1}$ H NMR Spectrum of 4-(1H-benzo[d]imidazol-2-yl)phenol (2u) $(300 \mathrm{MHz}$, DMSO)

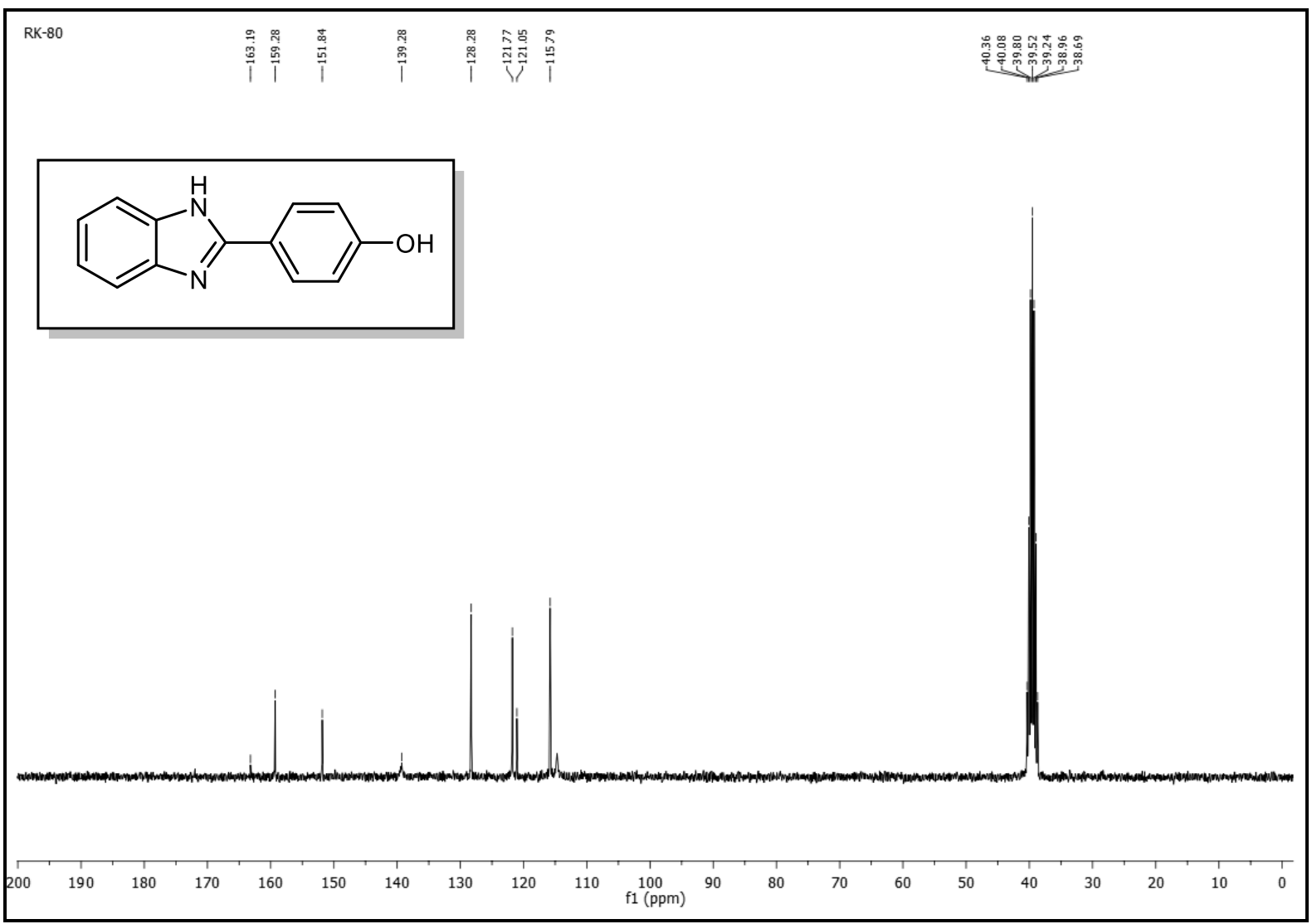

Fig. S49 ${ }^{13}$ C NMR Spectrum of 4-(1H-benzo[ $\left.d\right]$ imidazol-2-yl)phenol (2u) (75 MHz, DMSO) 


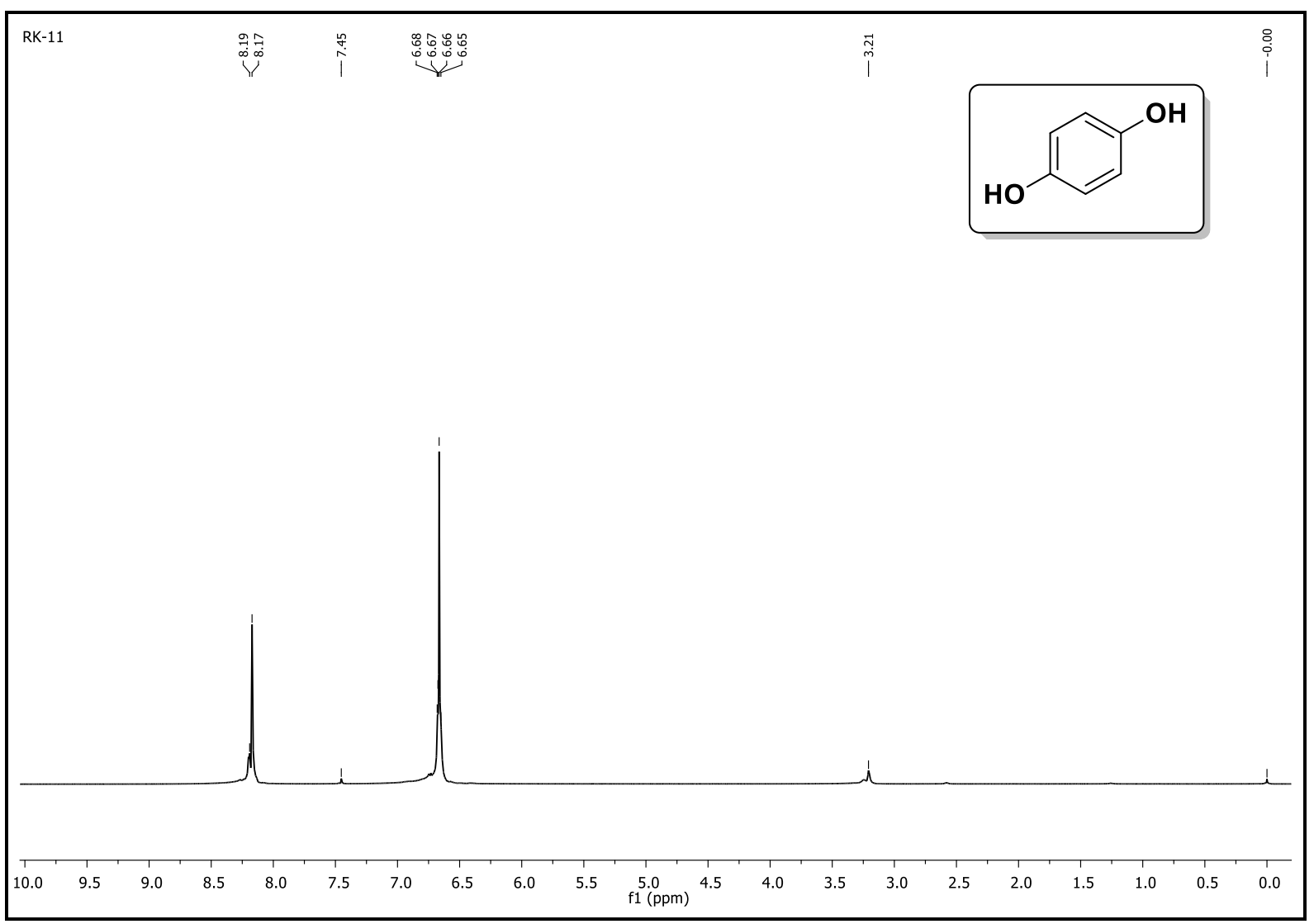

Fig. S50 ${ }^{1} \mathrm{H}$ NMR Spectrum of hydroquinone (2v) $\left(300 \mathrm{MHz}, \mathrm{CDCl}_{3}\right)$

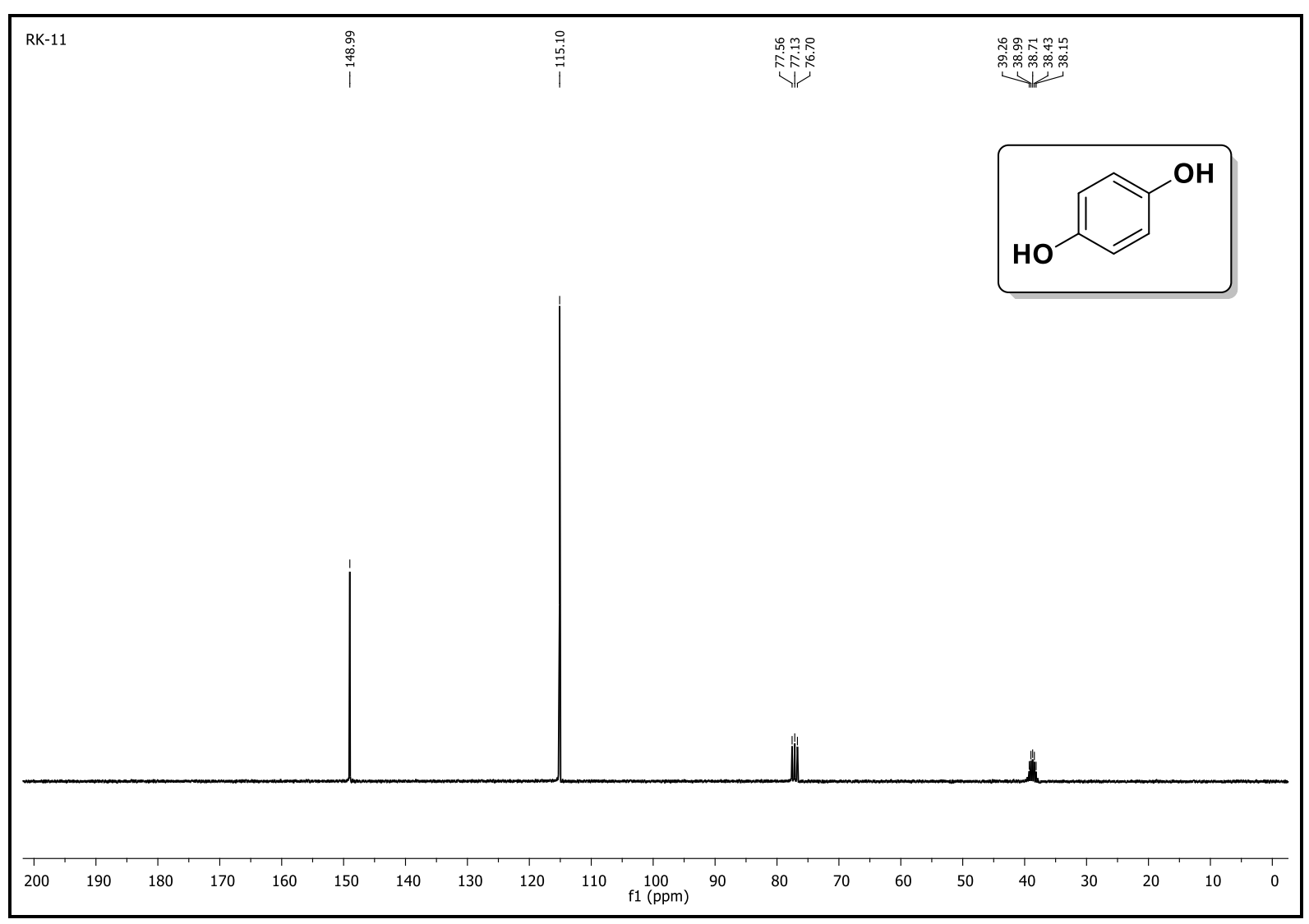

Fig. $\mathbf{S 5 1}{ }^{13} \mathrm{C}$ NMR Spectrum of hydroquinone (2v) $\left(75 \mathrm{MHz}, \mathrm{CDCl}_{3}\right)$ 


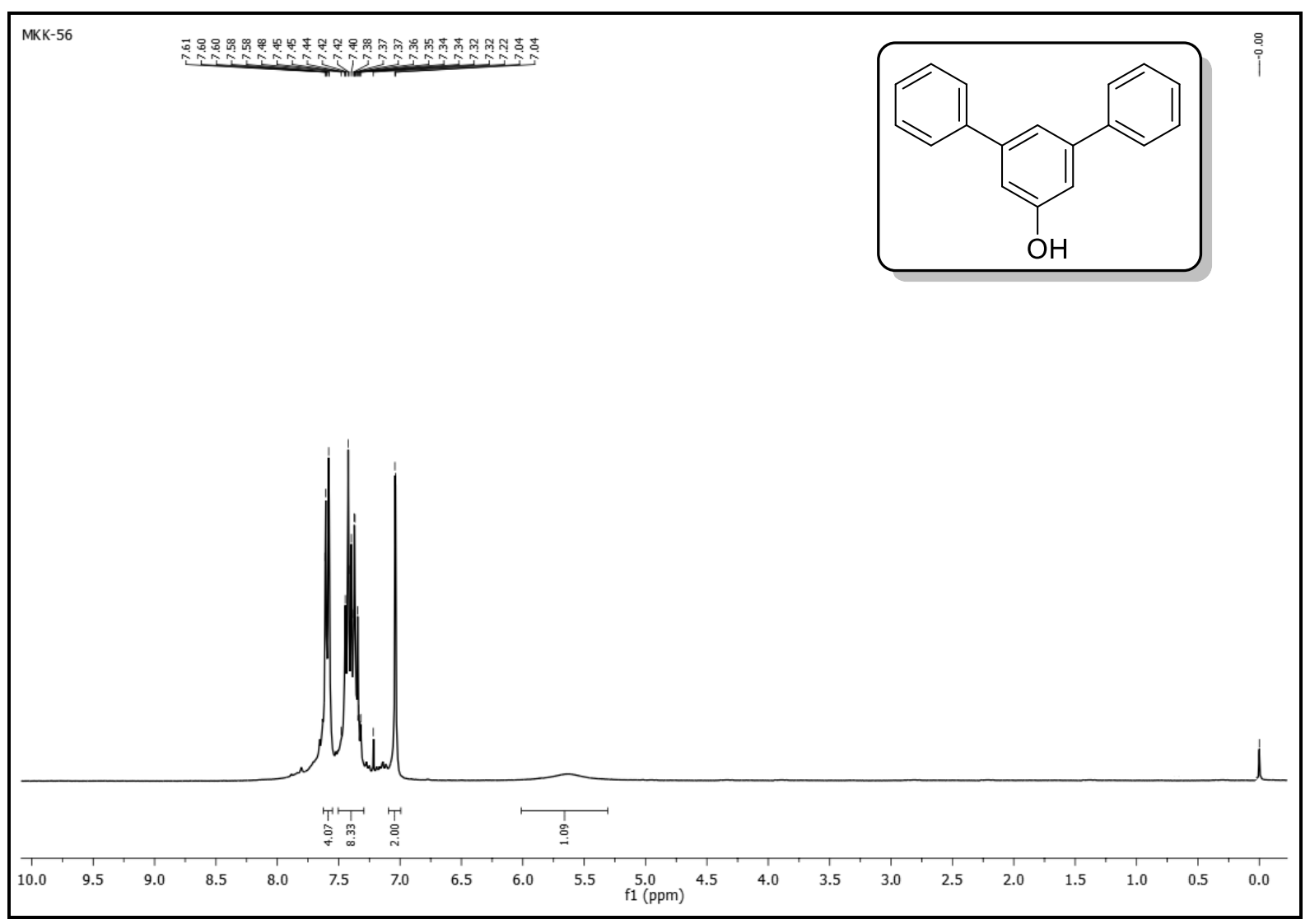

Fig. S52 ${ }^{13} \mathrm{C}$ NMR Spectrum of $\left[1,1^{\prime}: 3^{\prime}, 1^{\prime}\right.$-terphenyl]-5'-ol (2w) $\left(300 \mathrm{MHz}, \mathrm{CDCl}_{3}\right)$

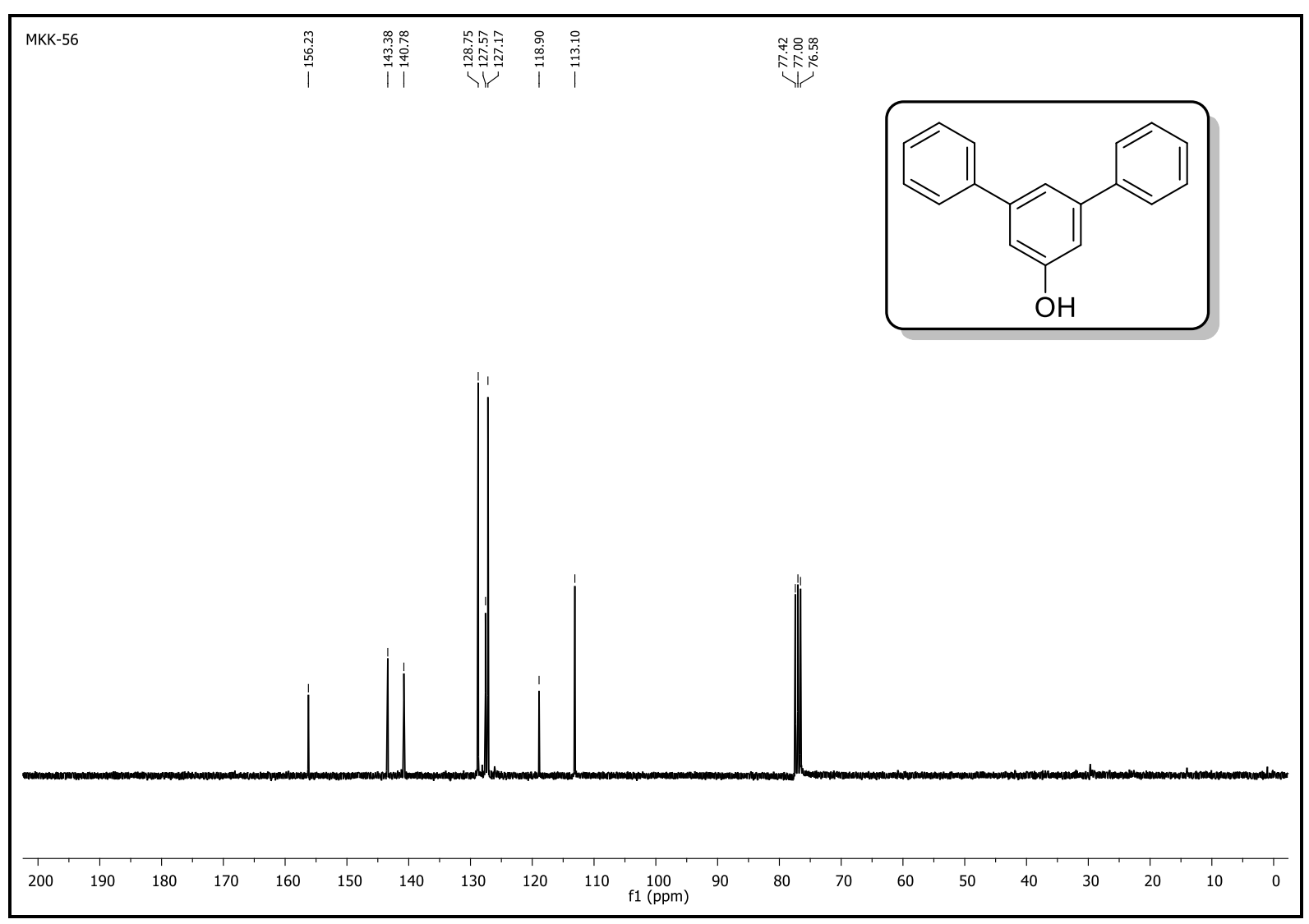

Fig. S53 ${ }^{13} \mathrm{C}$ NMR Spectrum of [1,1':3',1"-terphenyl]-5'-ol (2w) $\left(75 \mathrm{MHz}, \mathrm{CDCl}_{3}\right)$ 


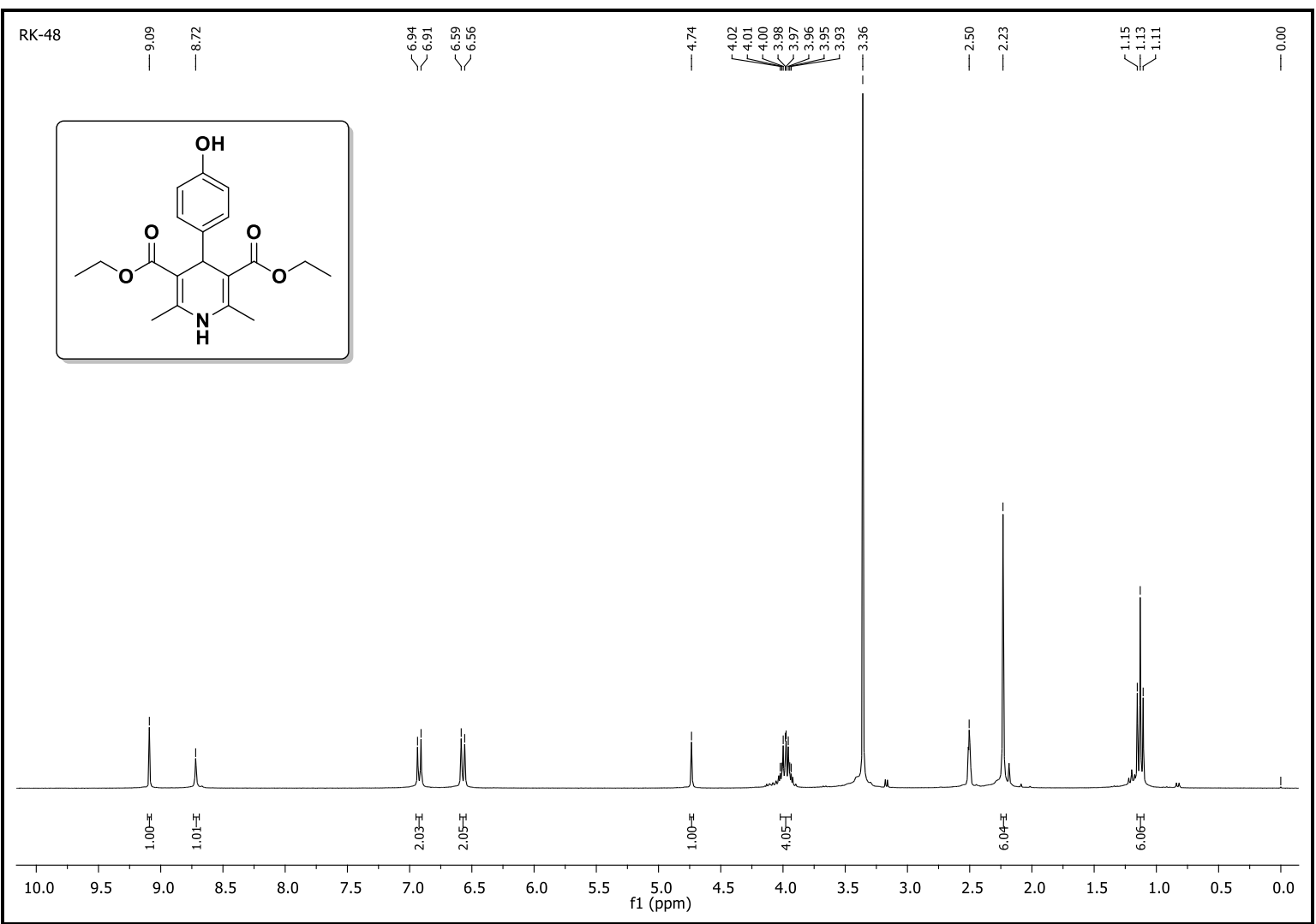

Fig. S54 ${ }^{1}$ H NMR Spectrum of diethyl 4-(4-hydroxyphenyl)-2,6-dimethyl-1,4-dihydropyridine-3,5dicarboxylate (2x) $\left(300 \mathrm{MHz}, \mathrm{CDCl}_{3}\right)$

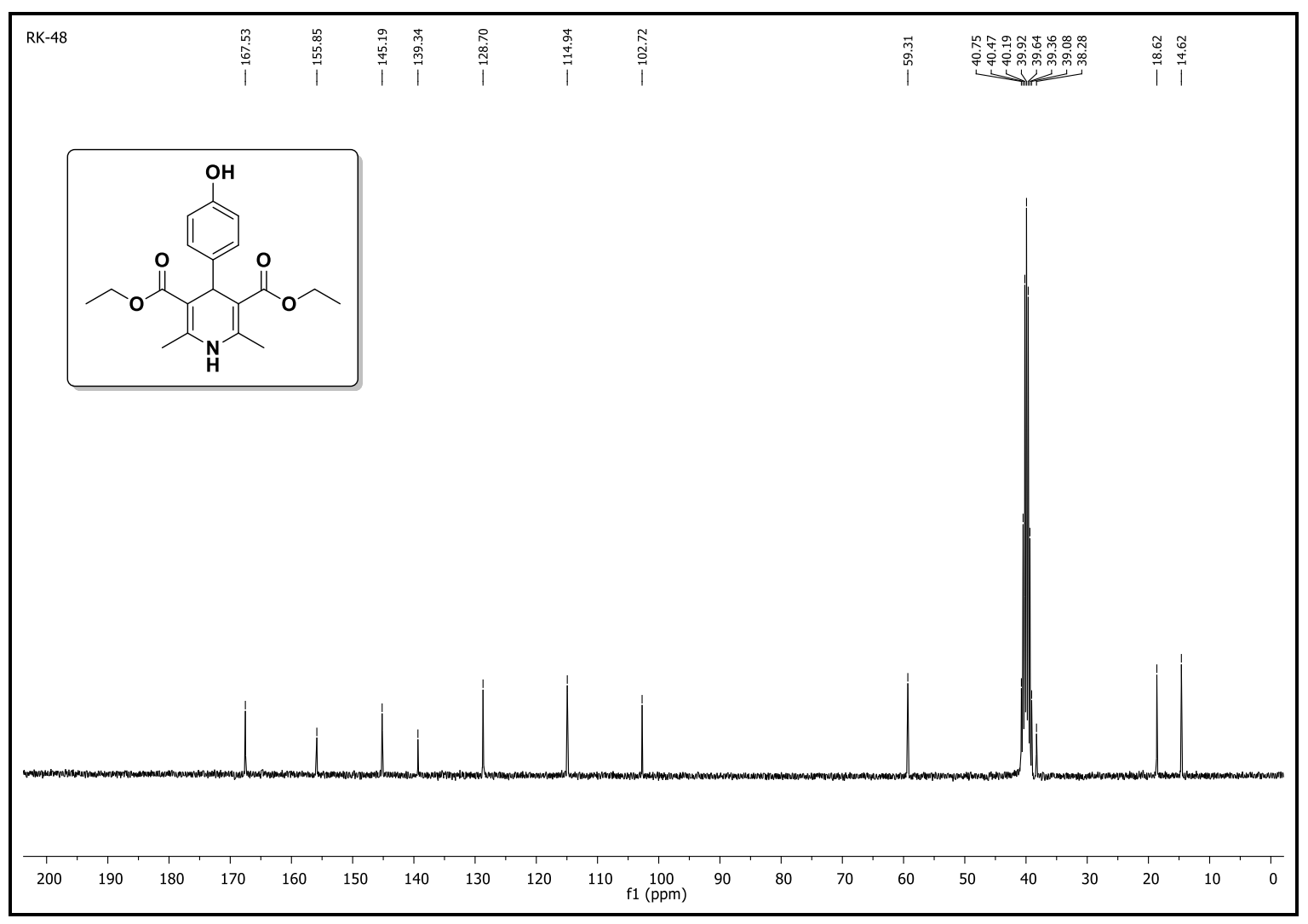

Fig. S55 ${ }^{13}$ C NMR Spectrum of diethyl 4-(4-hydroxyphenyl)-2,6-dimethyl-1,4-dihydropyridine-3,5dicarboxylate $(\mathbf{2 x})\left(75 \mathrm{MHz}, \mathrm{CDCl}_{3}\right)$ 


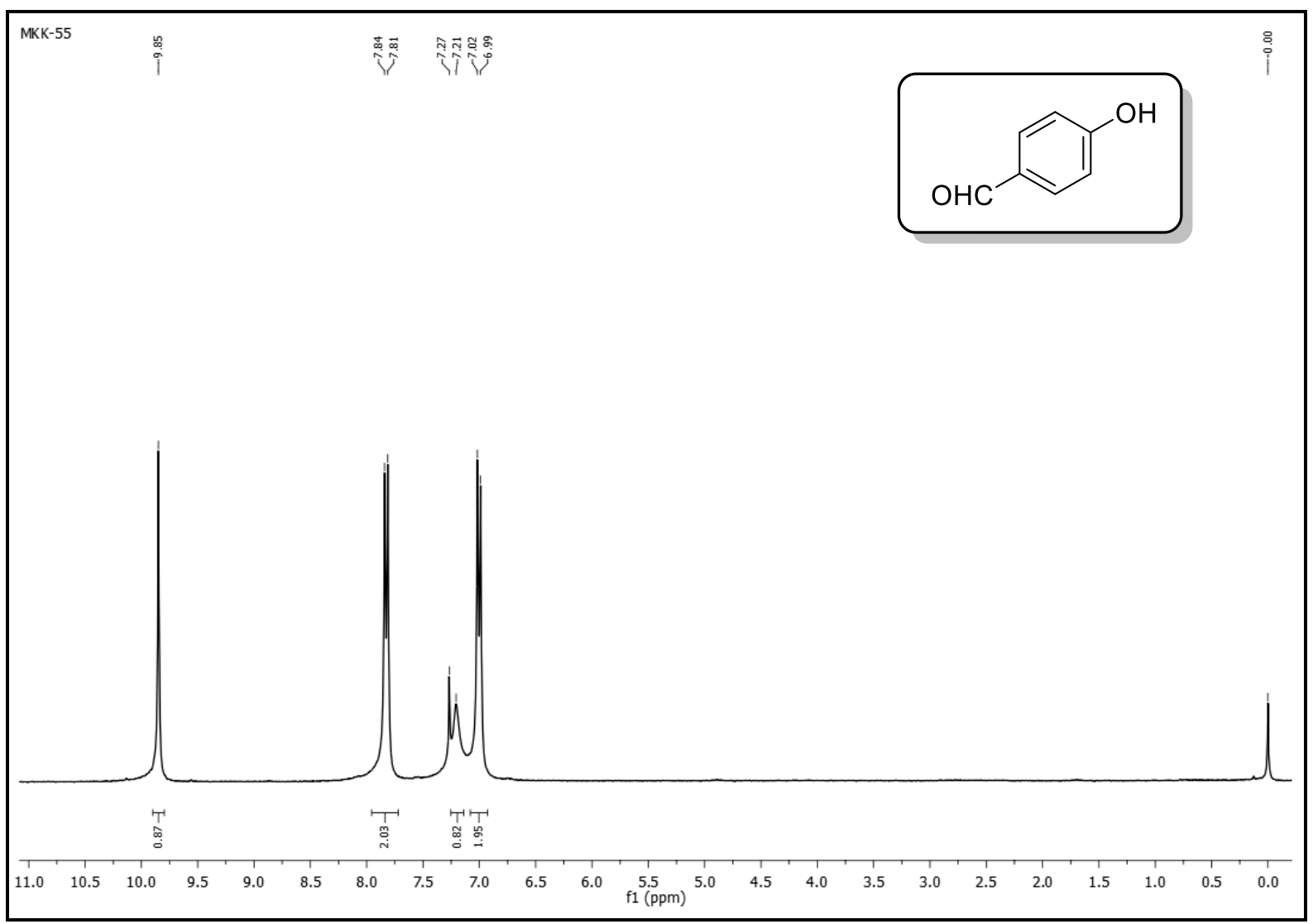

Fig. $\mathbf{S 5 6}{ }^{1} \mathrm{H}$ NMR Spectrum of 4-hydroxybenzaldehyde ( 2 y) $\left(300 \mathrm{MHz}, \mathrm{CDCl}_{3}\right)$

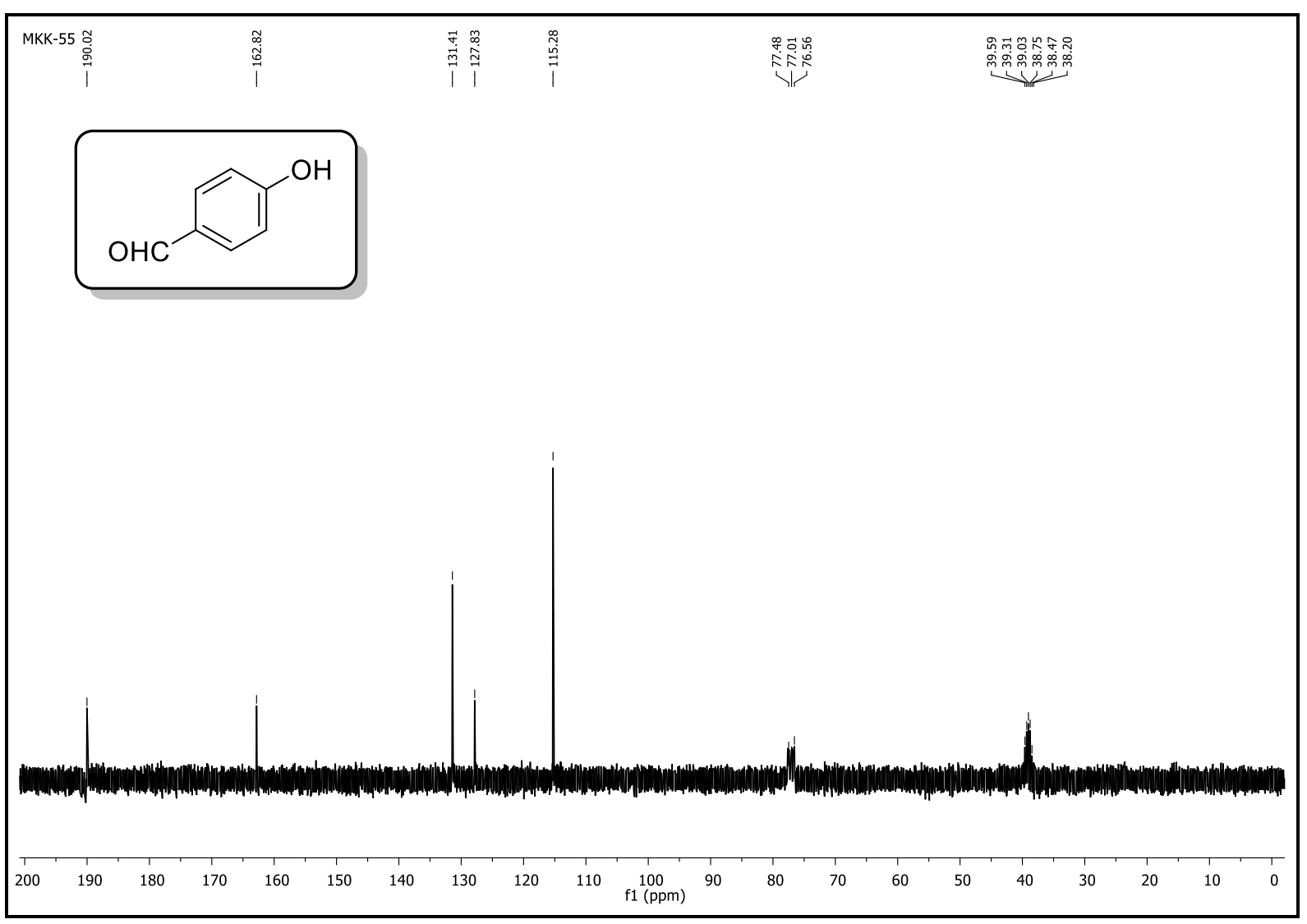

Fig. $\mathbf{S 5 7}{ }^{13} \mathrm{C}$ NMR Spectrum of 4-hydroxybenzaldehyde ( $\left.2 \mathrm{y}\right)\left(75 \mathrm{MHz}, \mathrm{CDCl}_{3}\right)$ 


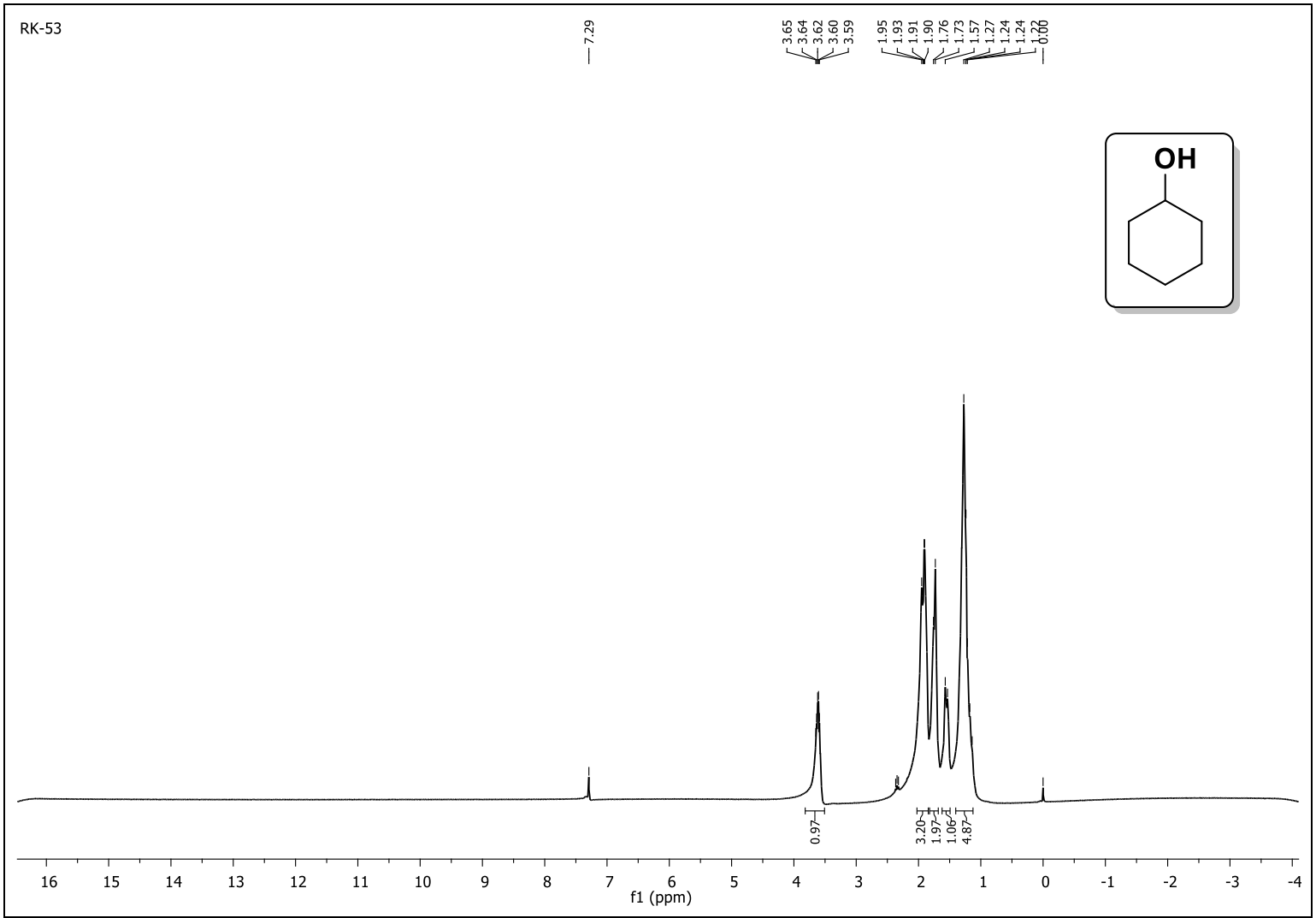

Fig. S58 ${ }^{1} \mathrm{H}$ NMR Spectrum of Cyclohexanol (2z) $\left(300 \mathrm{MHz}, \mathrm{CDCl}_{3}\right)$

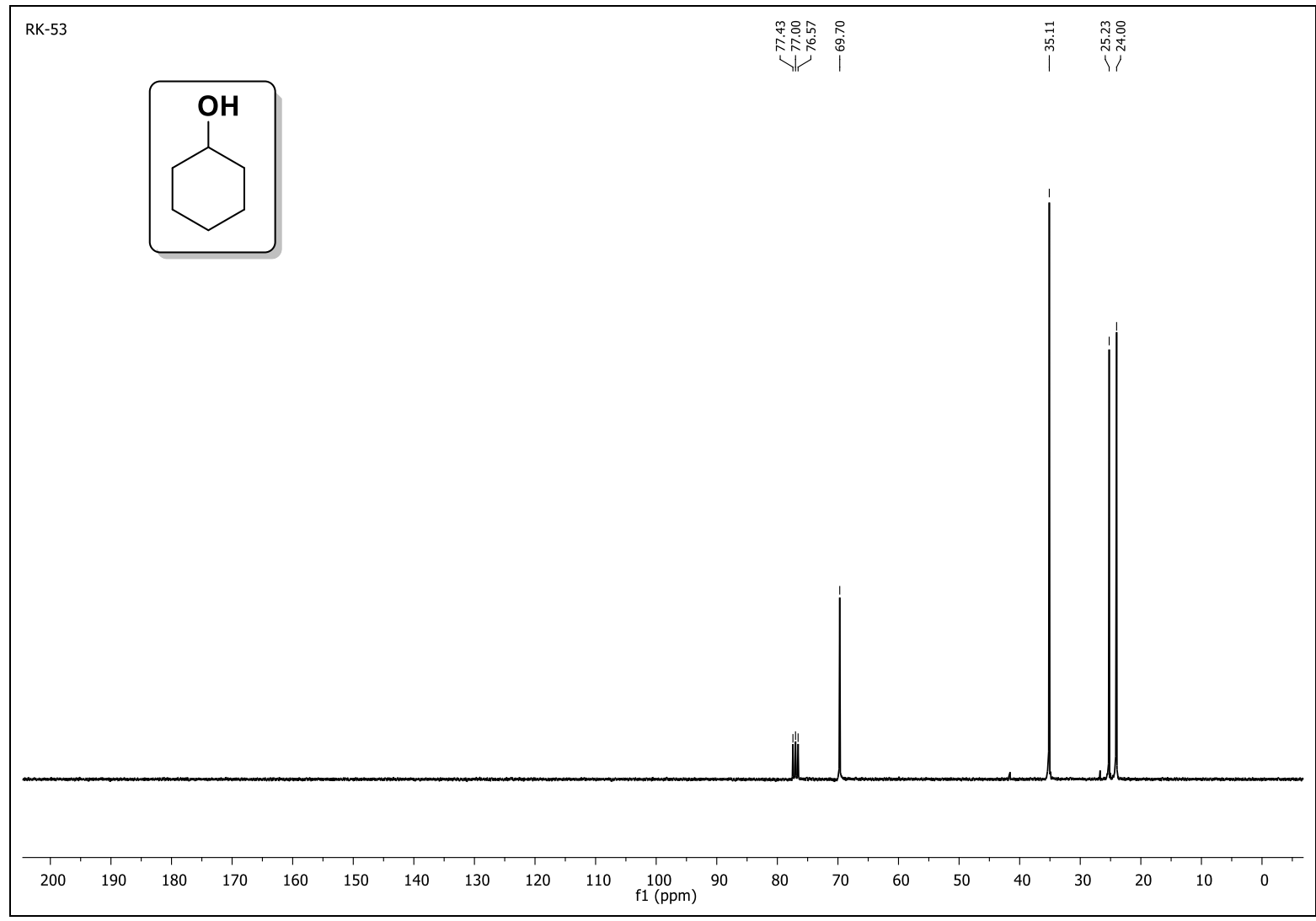

Fig. S59 ${ }^{13} \mathrm{C}$ NMR Spectrum of Cyclohexanol (2z) $\left(75 \mathrm{MHz}, \mathrm{CDCl}_{3}\right)$ 